\title{
DOUBLING CONSTRUCTIONS AND TENSOR PRODUCT L-FUNCTIONS: THE LINEAR CASE
}

\author{
YUANQING CAI, SOLOMON FRIEDBERG, DAVID GINZBURG, AND EYAL KAPLAN
}

\begin{abstract}
We present an integral representation for the tensor product $L$-function of a pair of automorphic cuspidal representations, one of a classical group, the other of a general linear group. Our construction is uniform over all classical groups, and is applicable to all cuspidal representations; it does not require genericity. The main new ideas of the construction are the use of generalized Speh representations as inducing data for the Eisenstein series and the introduction of a new (global and local) model, which generalizes the Whittaker model. Here we consider linear groups, but our construction also extends to arbitrary degree metaplectic coverings; this will be the topic of an upcoming work.
\end{abstract}

\section{INTRODUCTION}

One of the pillars of the Langlands program is the study of global automorphic $L$-functions as mediating agents in the framework of functoriality. The analytic properties of $L$-functions for representations of classical groups twisted by representations of general linear groups played a central role in the proof of functoriality for classical groups by Cogdell et al. [CKPSS04]. That proof relied on the Converse Theorem of Cogdell and Piatetski-Shapiro [CPS94, CPS99]: strong analytic properties of the twisted $L$-functions imply automorphicity. Cogdell et al. only considered globally generic representations - those affording a Whittaker-Fourier coefficient because constructions of the $L$-functions, either using the Langlands-Shahidi method or the Rankin-Selberg method, were limited to such representations.

On the other hand, Piatetski-Shapiro and Rallis [PSR87a] introduced a different type of global integral which represents the standard $L$-function for any classical group. Their construction is advantageous in two important aspects. First, it presents a unified approach to integral representations of these $L$-functions, comparable to the uniformity of the LanglandsShahidi method. Second, it is applicable to any cuspidal automorphic representation on the classical group. Previously known integrals unfolded to a special model, afforded by some but not all cuspidal automorphic representations, most notably the Whittaker model. In contrast, the construction of [PSR87a], now known as the doubling method, unfolded to an integral involving a global matrix coefficient on the classical group, which is always nontrivial for some choice of data, and for decomposable data can be expressed as the (infinite) product of local matrix coefficients. On the downside, these constructions were limited to the standard representation, or its twists by characters. Thus they did not provide enough information to be used in concert with the Converse Theorem to establish functoriality for non-generic automorphic representations.

2010 Mathematics Subject Classification. Primary 11F70; Secondary 11F55, 11F66, 22E50, $22 \mathrm{E} 55$.

Key words and phrases. Doubling method, Eisenstein series, Whittaker-Speh-Shalika representation, Rankin-Selberg $L$-function, non-generic automorphic representation, unipotent orbit, metaplectic cover.

This research was supported by the ERC, StG grant number 637912 (Cai), by the BSF, grant number 2012019 (Friedberg and Ginzburg), by the NSF, grant numbers DMS-1500977 and DMS-1801497 (Friedberg), and by the Israel Science Foundation, grant number 421/17 (Kaplan). 
Here we describe a new construction, which extends the doubling method, to provide integral representations for arbitrary automorphic cuspidal representations of classical groups twisted by automorphic cuspidal representations of arbitrary rank general linear groups. Our integrals inherit the benefits of the doubling method in that the construction is uniform across all classical groups and applies to all cuspidal automorphic representations (as opposed to only globally generic ones), but in sharp contrast with the doubling method, we are not limited to rank-one twists.

This paper removes a fundamental obstruction to extending the functoriality results of Cogdell et al. [CKPSS04] to any automorphic cuspidal representation. Although such liftings also follow from the work of Arthur, an independent proof is of high interest. In a forthcoming work we further develop the global and local theory, analyze the local integrals over both non-archimedean and archimedean fields and define $\gamma$-, $L$ - and $\epsilon$-factors, along the lines of the work of Lapid and Rallis [LR05] on the original doubling method (see also [Gan12, Yam14]). We use these results to construct a functorial lift of $\pi$ to $\mathrm{GL}_{N}(\mathbb{A})$ using the Converse Theorem (see $[\mathrm{CFK}]$ ).

Let $F$ be a number field with a ring of adeles $\mathbb{A}$, and $G$ be a split classical group. Let $\pi$ and $\tau$ be irreducible cuspidal automorphic representations of $G(\mathbb{A})$ and $G_{k}(\mathbb{A})$, respectively. We construct an Eisenstein series $E(h ; f, s)$ on $H(\mathbb{A})$, where $H$ is an auxiliary classical group defined depending on $G$ and $k$. The inducing data of the Eisenstein series is a generalized Speh representation $\mathcal{E}_{\tau}$ attached to $\tau$. We choose a unipotent subgroup $U$ of $H$ and an automorphic character $\psi_{U}$ of $U$, such that $G \times G$ is embedded in the normalizer of $U$ and stabilizer of $\psi_{U}$. We consider the integral

$$
Z\left(s, \varphi_{1}, \varphi_{2}, f\right)=\int_{G(F) \times G(F) \backslash G(\mathbb{A}) \times G(\mathbb{A})} \varphi_{1}\left(g_{1}\right) \overline{\varphi_{2}\left({ }^{\iota} g_{2}\right)} E^{U, \psi_{U}}\left(\left(g_{1}, g_{2}\right) ; f, s\right) d g_{1} d g_{2} .
$$

Here $\varphi_{1}$ and $\varphi_{2}$ are cusp forms in the space of $\pi,{ }^{\iota}$ is an involution of $G$ and $E^{U, \psi_{U}}$ denotes the Fourier coefficient of the series with respect to $U$ and $\psi_{U}$. This is a "doubling construction" in the sense that the integral is over two copies of $G$ and for $k=1$, reproduces the doubling integral of [PSR87a] (the original doubling method was motivated by doubling in the context of quadratic forms).

The main result of this paper is the following.

Theorem A. The global integral (1.1) represents the global partial L-function $L^{S}(s, \pi \times \tau)$.

The function $L^{S}(s, \pi \times \tau)$ here is the product of local $L$-functions over all finite places of $F$ for which the local data are unramified. Theorem A follows by combining Theorem 1, identity (3.1), Theorem 21 and Theorem 29 below. Here we treat two cases in detail: $G=\mathrm{Sp}_{2 n}$ and $\mathrm{SO}_{2 n}$.

In a subsequent paper we elaborate on the details of this construction also for $\mathrm{SO}_{2 n+1}$ and split connected general spin groups of arbitrary rank (see $[\mathrm{CFK}]$ ). In this paper we do not treat these groups as they require additional work of a technical nature.

The novel ingredients of (1.1) compared to the doubling method of [PSR87a], are the usage of the specialized inducing data, namely the representation $\mathcal{E}_{\tau}$, and the replacement of the Eisenstein series there with its Fourier coefficient. Critically, it turns out that the representation $\mathcal{E}_{\tau}$ is supported on a sufficiently small unipotent orbit. The unfolding process leads us to introduce a new (global and local) model, which we call a Whittaker-Speh-Shalika model, since it generalizes the Whittaker and Shalika models for generalized Speh representations (see Definition 3 below). The nonvanishing of the appropriate Fourier coefficient of $\mathcal{E}_{\tau}$, as well as the vanishing properties of $\mathcal{E}_{\tau}$ that we use, were proved by Ginzburg [Gin03]; see also Jiang and Liu [JL13] for a detailed study of these representations in a global context. Then to deduce 
that the integral is Eulerian (Theorem 1) we establish multiplicity one results, at least over the unramified places.

One immediate consequence of Theorem $\mathrm{A}$ is that $L^{S}(s, \pi \times \tau)$ admits meromorphic continuation to the plane, see Theorem 30. This is of course a well-known result of Langlands (e.g., [Lan67, Lan76]), who established it by analyzing the constant term of the Eisenstein series. However, the constant term approach is not sufficient to handle local factors at the remaining places, nor to get the full analytic behavior necessary to apply the Converse Theorem. Local factors for irreducible generic representations are usually defined via Shahidi's celebrated method of local coefficients (e.g., [Sha90]). This method is not applicable in general to nongeneric representations, hence the aforementioned functoriality results [CKPSS04] were limited to generic ones. By contrast, the local version of our integrals may be used to define and study local $L$ - and $\epsilon$-factors at all places. In fact, the definition of local factors using integral representations may well be the only available analytic method for the general case. For further reference see, e.g., [PSR86, PSR87b, Ike92, HKS96, Ike99, LR05, Kap13b, Yam14]. Note that historically, for general linear groups this was the original definition, see [GJ72, JPSS83].

Our ideas and construction apply also to non-linear coverings. Starting with genuine representations $\pi$ and $\tau$ of certain covering groups of $G(\mathbb{A})$ and $G_{k}(\mathbb{A})$, we construct a similar global integral, the main difference being the rank of $H$, which now also depends on the parameters of the covering. We will describe this construction in a subsequent work. See also Gao [Gao18] for the extension of the constant term approach to covering groups.

For linear groups, the descent method was used to construct an explicit realization of an inverse to the functorial lift from globally generic representations of classical groups to $\mathrm{GL}_{N}$; see Ginzburg et al. [GRS99a, GRS99b, GRS11] and also Soudry [Sou05]. We expect to use the integrals developed here to extend the descent method to functorial lifts of arbitrary automorphic cuspidal representations, and also to obtain new descent constructions for covering groups.

The doubling method has had numerous important applications. We list several of these. Its strong relation to the theta correspondence, via the Siegel-Weil formula, has been studied in [KR94, HKS96, GS12, Yam14]; Böcherer and Schmidt [BS00] used the doubling method to construct standard $p$-adic $L$-functions for Siegel modular forms; recently, Eischen et. al. [EHLS] used this method to construct $p$-adic $L$-functions for unitary groups, completing the results of Harris et. al. [HLS06] (see also [HLS05]), which are part of a long-term project by these authors; and Garrett [Gar84] developed the doubling method in a classical framework. Among other works on the doubling method we mention [Tak97, Kim00].

The original doubling method was developed for classical groups of symplectic, orthogonal or unitary type [PSR87a, LR05], and later extended to several more cases including the double cover of the symplectic group by Gan [Gan12], and unitary groups of hermitian or skew-hermitian forms over division algebras in the work of Yamana [Yam14]. We expect similar extensions to be applicable here. Interestingly, the odd orthogonal case was excluded from [PSR87a] and was first treated only in [LR05] (for technical reasons). Also note that we develop the theory for connected groups, i.e., $\mathrm{SO}_{2 n}$ instead of $\mathrm{O}_{2 n}$. This is compatible with the theories of Langlands and Shahidi, which were formulated for connected groups, and with several other works on Rankin-Selberg integrals.

Earlier works on integral representations include [JS81, GPSR87, BF90, Gin90, JS90, BG92, Sou93, Tak14]. Recent works [GPSR97, GJRS11, JZ14] developed L-functions for tensor products of automorphic cuspidal representations of classical groups and general linear groups. In these works the Whittaker model was replaced by a pairing with a suitable auxiliary cuspidal 
representation. In particular, Jiang and Zhang [JZ14] extended the construction from cuspidal representations of $\mathrm{GL}_{k}$ to isobaric representations. The isobaric sum was previously considered in [GRS11], albeit in a less general context. We also mention two recent works by Soudry [Sou17, Sou18], who reduced local computations with non-generic data to the known generic case, in the context of the integrals of [GPSR97, GJRS11, JZ14]. By contrast, our approach to the (local) study of non-generic representations is to use the uniqueness of the pairing of an irreducible representation with its contragredient, which is true without any additional assumption (even over covering groups).

The rest of this work is organized as follows. In $\S 2$ we present the global construction, starting with the integral $(\S 2.1)$, then discuss the generalized Speh representation and its properties $(\S 2.2)$ and carry out the unfolding process ( $\S 2.3)$. The computation of the local integrals with unramified data is described in $\S 3$. The local integrals are presented in $\S 3.1$ and their computation is reduced to a similar computation on general linear groups, which is further reduced to a rank-1 case ( $\S 3.6)$. The latter integral is computed in $\S 3.7$ by, surprisingly enough, reducing it to the familiar Rankin-Selberg integrals of $\mathrm{GL}_{1} \times \mathrm{GL}_{k}$ and $\mathrm{GL}_{1} \times \mathrm{GL}_{2 k}$ from [JS81, JPSS83].

Acknowledgments. Part of this work was done while the fourth named author was a Zassenhaus Assistant Professor at The Ohio State University, under the supervision of Jim Cogdell. Eyal $^{1}$ wishes to express his gratitude to Jim for his kind encouragement and support.

\section{The global COnstruction}

2.1. The global integral. We introduce the general global integral. Let $n$ and $k$ denote two positive integers, $F$ be a number field with a ring of adeles $\mathbb{A}$, and $G$ be a split connected classical group of rank $n$. Let $\pi_{1}$ and $\pi_{2}$ denote two irreducible cuspidal automorphic representations of $G(\mathbb{A})$, and $\tau$ denote an irreducible cuspidal automorphic representation of $\mathrm{GL}_{k}(\mathbb{A})$.

Let $c=c(n)$ be the rank of the natural general linear group containing $G$, i.e., $c=2 n$ for $G=\mathrm{Sp}_{2 n}$ and $\mathrm{SO}_{2 n}$, and $c=2 n+1$ for $G=\mathrm{SO}_{2 n+1}$. Depending on $G$, we introduce another classical group $H$ of rank $k c$, on which we shall construct an Eisenstein series. For example if $G=\mathrm{Sp}_{2 n}, H=\mathrm{Sp}_{4 k n}$. Fix a Borel subgroup $B_{H}$ in $H$ and let $P=M_{P} \ltimes U_{P}$ denote a maximal standard parabolic subgroup of $H$ with a Levi part $M_{P} \cong \mathrm{GL}_{k c}$, i.e., a so-called Siegel parabolic subgroup. The precise definitions of $H$ and $P$ will be given near the end of this section.

The key building block in our construction is a residue representation $\mathcal{E}_{\tau}$ of $\mathrm{GL}_{k c}(\mathbb{A})$, which we call a Whittaker-Speh-Shalika representation of type $(k, c)$. In this work it is the generalized Speh representation corresponding to $c$ copies of $\tau$. The definition and construction are detailed in $\S 2.2$ below. Its fundamental properties are that it is supported on a sufficiently small unipotent orbit (in the sense of [Gin06]), and on this orbit it supports a $(k, c)$ functional $\Lambda$. This functional is "almost decomposable" (see Claim 4). These properties are crucial for the unfolding argument and proof that the global integral is "almost" an Euler product (see (3.1)). Explicitly, if $\varphi$ belongs to the space of $\mathcal{E}_{\tau}$,

$$
\Lambda(\varphi)=\int_{V_{\left(c^{k}\right)}(F) \backslash V_{\left(c^{k}\right)}(\mathbb{A})} \varphi(v) \psi^{-1}\left(\operatorname{tr}\left(\sum_{i=1}^{k-1} v_{i, i+1}\right)\right) d v,
$$

where $V_{\left(c^{k}\right)}$ is a subgroup of upper triangular matrices, which is the unipotent radical of the parabolic subgroup of $\mathrm{GL}_{k c}$ corresponding to the partition $\left(c^{k}\right), v_{1,2}, \ldots, v_{k-1, k}$ are the $c \times c$

\footnotetext{
${ }^{1}$ Eyal dedicates his part of the work to his beloved Sophie Kaplan who passed away unexpectedly a few weeks before the submission of the first version of this work.
} 
blocks above the main diagonal of $v($ see $\S 2.2)$ and $\operatorname{tr}$ is the trace map. For example when $c=1$, this is the usual Whittaker-Fourier coefficient and $\mathcal{E}_{\tau}$ is simply $\tau$, which is known to be globally generic, i.e., supports a $(k, 1)$ functional. Note that for $c>1$ we do not claim or expect arbitrary cuspidal automorphic representations of $\mathrm{GL}_{k c}(\mathbb{A})$ to be of type $(k, c)$, this is a special property enjoyed by the representations $\mathcal{E}_{\tau}$; see also Remark 14 for a local discussion.

Let $K_{H}$ be a maximal compact subgroup of $H$ which is in a "good position" with respect to the maximal torus of $B_{H}$ (see e.g., [MW95, § I.1.4]). Form the Eisenstein series $E(h ; f, s)$ on $H(\mathbb{A})$, attached to the induced representation

$$
\operatorname{Ind}_{P(\mathbb{A})}^{H(\mathbb{A})}\left(\mathcal{E}_{\tau} \delta_{P}^{s}\right)
$$

Here $\delta_{P}$ is the modulus character of $P$ (throughout, induction is normalized). By definition, for $\operatorname{Re}(s) \gg 0$,

$$
E(h ; f, s)=\sum_{\gamma \in P(F) \backslash H(F)} f(\gamma h, s), \quad h \in H(\mathbb{A}),
$$

where $f(h, s)$ is a standard section, i.e., a section whose restriction to $K_{H}$ is independent of $s$. We will use a certain Fourier coefficient of this series.

To describe this coefficient, let $Q=M \ltimes U$ be a standard parabolic subgroup of $H$, whose Levi part $M$ is isomorphic to $k-1$ copies of $\mathrm{GL}_{c}$ multiplied by a split classical group of rank $c$. The subgroup $Q$ is uniquely defined given $k$ and the type of $H$. E.g., for $G=\mathrm{Sp}_{2 n}, M=\mathrm{GL}_{2 n} \times \ldots \times$ $\mathrm{GL}_{2 n} \times \mathrm{Sp}_{4 n}$. Recall that unipotent orbits for classical groups are indexed by (certain) partitions (see e.g., [Spa82, Car93, CM93]). Consider the unipotent orbit

$$
\left((2 k-1)^{c} 1^{c}\right)
$$

associated with the group $H$. It follows from Collingwood and McGovern [CM93] that this is a well-defined orbit for every group $H$ (for $\mathrm{Sp}_{2 n}$, odd numbers occur with even multiplicity, in the orthogonal cases this is clear since there are no even parts), and that the stabilizer of this orbit over an algebraically closed field contains the group $G \times G$. From [Gin06] we deduce that a Fourier coefficient associated with this orbit can be constructed along $U$, and an automorphic character $\psi_{U}$ of $U(\mathbb{A})$ can be defined such that its stabilizer inside $M(\mathbb{A})$ contains $G(\mathbb{A}) \times G(\mathbb{A})$. For an example of $U, \psi_{U}$ and the embedding $\left(g_{1}, g_{2}\right): G(\mathbb{A}) \times G(\mathbb{A}) \rightarrow M(\mathbb{A})<H(\mathbb{A})$ in the cases of $\mathrm{Sp}_{2 n}$ and $\mathrm{SO}_{2 n}$ see $\S 2.3$. For brevity, we denote the identity element of $G$ by 1 in the embedding, e.g., write $(1, g)$.

The global integral we consider is

$$
Z\left(s, \varphi_{1}, \varphi_{2}, f\right)=\int_{G(F) \times G(F) \backslash G(\mathbb{A}) \times G(\mathbb{A})} \int_{U(F) \backslash U(\mathbb{A})} \varphi_{1}\left(g_{1}\right) \overline{\varphi_{2}\left({ }^{\iota} g_{2}\right)} E\left(u\left(g_{1}, g_{2}\right) ; f, s\right) \psi_{U}(u) d u d g_{1} d g_{2} .
$$

Here $\varphi_{i}$ is a cusp form in the space of $\pi_{i},\left(g_{1}, g_{2}\right)$ is the embedding and $\iota$ is a certain involution of $G$ (see below). The integral converges absolutely for $\operatorname{Re}(s) \gg 0$ and admits meromorphic continuation to the whole complex plane; this follows from the rapid decay of cusp forms, moderate growth of the Eisenstein series and the meromorphic continuation of the Eisenstein series.

Let $L=(G \times G) U$. It is a subgroup of $Q$. The action of $L(F)$ on the right on the homogeneous space $P(F) \backslash H(F)$ has a unique open orbit. Let $\delta \in H(F)$ be a representative for this orbit. The involution $\iota$ is chosen such that $\delta\left(g,{ }^{\iota} g\right) \delta^{-1} \in M_{P}(\mathbb{A})$ for all $g \in G(\mathbb{A})$. Denote $U_{0}=U \cap U_{P}$. 
Also let

$$
\left\langle\varphi_{1}, \varphi_{2}\right\rangle=\int_{G(F) \backslash G(\mathbb{A})} \varphi_{1}(g) \overline{\varphi_{2}(g)} d g
$$

be the standard inner product on $G(\mathbb{A})$. Refer to $\S 2.3$ for the concrete choices of $\iota$ and $\delta$ (for $\mathrm{Sp}_{2 n}$ see (2.10) and (2.18)).

In the following theorem we state the basic properties of the integral.

Theorem 1. The integral $Z\left(s, \varphi_{1}, \varphi_{2}, f\right)$ is absolutely convergent for $\operatorname{Re}(s) \gg 0$ and admits meromorphic continuation to the plane. It is not identically zero only if $\pi_{1}=\pi_{2}=\pi$. In this case, for $\operatorname{Re}(s) \gg 0$ it is equal to

$$
\int_{G(\mathbb{A})} \int_{U_{0}(\mathbb{A})}\left\langle\varphi_{1}, \pi(g) \varphi_{2}\right\rangle f_{W\left(\mathcal{E}_{\tau}\right)}\left(\delta u_{0}\left(1,{ }^{\iota} g\right), s\right) \psi_{U}\left(u_{0}\right) d u_{0} d g .
$$

Here $f_{W\left(\mathcal{E}_{\tau}\right)}$ is the composition of the section and the unique functional $\Lambda$ attached to $\mathcal{E}_{\tau}$ : for any $s \in \mathbb{C}$ and $h \in H(\mathbb{A})$,

$$
f_{W\left(\mathcal{E}_{\tau}\right)}(h, s)=\int_{V_{\left(c^{k}\right)}(F) \backslash V_{\left(c^{k}\right)}(\mathbb{A})} f(v h, s) \psi^{-1}\left(\operatorname{tr}\left(\sum_{i=1}^{k-1} v_{i, i+1}\right)\right) d v .
$$

We prove the main identity (2.1) for $\mathrm{Sp}_{2 n}$ in $\S$ 2.3. The proof for $\mathrm{SO}_{2 n}$ is similar, and the changes that are needed for this group are described in Remark 20 below. Also note that, while we do not provide details for other groups in this work, Theorem 1 is also valid for $\mathrm{SO}_{2 n+1}$, and (with minor changes) for split connected general spin groups of even or odd rank.

As explained in the introduction, here we describe in detail the cases of the split groups $\operatorname{Sp}_{2 n}$ and $\mathrm{SO}_{2 n}$. For concreteness, $\mathrm{Sp}_{2 n}$ is defined as the subgroup of matrices $g \in \mathrm{SL}_{2 n}$ such that ${ }^{t} g\left({ }_{-J_{n}}{ }^{J_{n}}\right) g=\left({ }_{-J_{n}}{ }^{J_{n}}\right)$, where ${ }^{t} g$ is the transpose of $g$ and $J_{n}$ is the $n \times n$ permutation matrix having 1 on its anti-diagonal. Also define $\mathrm{SO}_{2 n}=\left\{g \in \mathrm{SL}_{2 n}:{ }^{t} g J_{2 n} g=J_{2 n}\right\}$.

Put $c=2 n$. For $G=\mathrm{Sp}_{2 n}$ let $H=\mathrm{Sp}_{2 k c}$, if $G=\mathrm{SO}_{2 n}$ take $H=\mathrm{SO}_{2 k c}$. Regarding $H$ as a subgroup of $\mathrm{GL}_{2 k c}$, choose the Borel subgroup $B_{H}=B_{\mathrm{GL}_{2 k c}} \cap H$, where $B_{\mathrm{GL}_{2 k c}}<\mathrm{GL}_{2 k c}$ is the subgroup of upper triangular invertible matrices, and similarly $B_{G}$ for $G<\mathrm{GL}_{c}$. This already fixes $P$ unless $H=\mathrm{SO}_{2 k c}$, then we choose $P$ with $M_{P}=\left\{\operatorname{diag}\left(g, J_{k c}{ }^{t} g^{-1} J_{k c}\right): g \in \mathrm{GL}_{k c}\right\}$.

The doubling construction can also be described for general linear groups extending the case $k=1$ of [PSR87a, $\S 4.2$ ]. One must divide by the center and handle convergence (as in [PSR87a, $\S 4.2]$ ). We omit the details, since we will only be using these integrals locally, for the purpose of computing the integrals for classical groups with unramified data.

2.2. Whittaker-Speh-Shalika Representations. We present the family of representations $\mathcal{E}_{\tau}$ used in $\S 2.1$ to define the Eisenstein series.

In the group $\mathrm{GL}_{l}$, write $B_{\mathrm{GL}_{l}}=T_{\mathrm{GL}_{l}} \ltimes N_{\mathrm{GL}_{l}}$ where $T_{\mathrm{GL}_{l}}$ is the diagonal torus. For a composition $\left(l_{1}, \ldots, l_{r}\right)$ of $l, P_{\left(l_{1}, \ldots, l_{r}\right)}=M_{\left(l_{1}, \ldots, l_{r}\right)} \ltimes V_{\left(l_{1}, \ldots, l_{r}\right)}$ denotes the standard parabolic subgroup of $\mathrm{GL}_{l}$ whose Levi part $M_{\left(l_{1}, \ldots, l_{r}\right)}$ is isomorphic to $\mathrm{GL}_{l_{1}} \times \ldots \times \mathrm{GL}_{l_{r}}$. (We recall that a composition of a positive integer $l$ is an ordered sequence of positive integers summing to $l$.) Also let $C_{l}$ be the center of $\mathrm{GL}_{l}$, denote the additive group of $l \times l^{\prime}$ matrices by $\mathrm{Mat}_{l \times l^{\prime}}$, and set $\mathrm{Mat}_{l}=\mathrm{Mat}_{l \times l}$.

Recall that the unipotent orbits of $\mathrm{GL}_{l}$ are in bijection with the partitions of $l$, and for such a partition there is a corresponding unipotent subgroup and a set of generic characters (see [Gin06, § 2] for these definitions). 
Let $k$ and $c$ be positive integers. The unipotent subgroup corresponding to the orbit $\left(k^{c}\right)$ is $V_{\left(c^{k}\right)}$. Fix a nontrivial character $\psi$ of $F \backslash \mathbb{A}$. Denote a matrix $v \in V_{\left(c^{k}\right)}$ by $v=\left(v_{i, j}\right)_{1 \leq i, j \leq k}$, where $v_{i, j} \in \operatorname{Mat}_{c}$. For an automorphic function $\varphi$ on $\mathrm{GL}_{k c}(F) \backslash \mathrm{GL}_{k c}(\mathbb{A})$, consider the integral

$$
\Lambda(\varphi)=\int_{V_{\left(c^{k}\right)}(F) \backslash V_{\left(c^{k}\right)}(\mathbb{A})} \varphi(v) \psi^{-1}(v) d v,
$$

where $\psi$ is the character of $V_{\left(c^{k}\right)}$ defined by

$$
\psi(v)=\psi\left(\operatorname{tr}\left(\sum_{i=1}^{k-1} v_{i, i+1}\right)\right) .
$$

This is a Fourier coefficient corresponding to the orbit $\left(k^{c}\right)$, and we call it a Whittaker-SpehShalika coefficient.

Example 2. In particular when $c=1$,

$$
\Lambda(\varphi)=\int_{N_{\mathrm{GL}_{k}}(F) \backslash N_{\mathrm{GL}_{k}}(\mathbb{A})} \varphi(v) \psi^{-1}\left(\sum_{i=1}^{k-1} v_{i, i+1}\right) d v
$$

is the well-known Whittaker-Fourier coefficient. An automorphic representation $\rho$ of $\mathrm{GL}_{k}(\mathbb{A})$ is globally generic when this functional is not identically zero on the elements $\varphi$ in the space of $\rho$. As we will see below, the representation $\mathcal{E}_{\tau}$ is defined for $c=1$ to be $\tau$ itself. Since $\tau$ is cuspidal, by [JL70, Sha74, PS75] it is globally generic.

Definition 3. An irreducible automorphic representation $\rho$ of $\mathrm{GL}_{k c}(\mathbb{A})$ is a Whittaker-SpehShalika representation of type $(k, c)$, or briefly a $(k, c)$ representation, if the following holds.

(1) The Fourier coefficient $\Lambda(\varphi)$ does not vanish identically on the space of $\rho$, and moreover, for all unipotent orbits greater than or non-comparable with $\left(k^{c}\right)$, all corresponding Fourier coefficients are zero for all choices of data.

(2) Let $\rho_{\nu}$ denote the irreducible constituent of $\rho$ at a finite place $\nu$, and assume $\rho_{\nu}$ is unramified. Then for all unipotent orbits greater than or non-comparable with $\left(k^{c}\right)$, the corresponding twisted Jacquet module of $\rho_{\nu}$ vanishes (i.e., the local analogue of (1) holds). Moreover, $\operatorname{Hom}_{V_{\left(c^{k}\right)}\left(F_{\nu}\right)}\left(\rho_{\nu}, \psi_{\nu}\right)$ is one-dimensional, where $\psi_{\nu}$ is given by $(2.3)$.

In the notation of [Gin06], condition (1) may be written as $\mathcal{O}_{\mathrm{GL}_{k c}}(\rho)=\left(k^{c}\right)$. The local vanishing properties of $\rho_{\nu}$ in the definition imply the global vanishing by a local-global principle (see e.g., [JR92, Proposition 1]). In the opposite direction, the nonvanishing of the global functional (2.2) implies $\operatorname{Hom}_{V_{\left(c^{k}\right)}\left(F_{\nu}\right)}\left(\rho_{\nu}, \psi_{\nu}\right) \neq 0$ for all $\nu$ (not only the unramified places), because (in general) the global functional gives rise to nonzero local functionals at all places.

For a unitary continuous character $\eta: F^{*} \backslash \mathbb{A}^{*} \rightarrow \mathbb{C}$, let $L^{2}\left(\mathrm{GL}_{k c}(F) \backslash \mathrm{GL}_{k c}(\mathbb{A}), \eta\right)$ be the space of measurable $L^{2}$-functions $\varphi: \mathrm{GL}_{k c}(F) \backslash \mathrm{GL}_{k c}(\mathbb{A}) \rightarrow \mathbb{C}$ such that $\varphi(z g)=\eta(z) \varphi(g)$ for all $z \in C_{k c}(\mathbb{A})$. The group $\mathrm{GL}_{k c}(\mathbb{A})$ acts on $L^{2}\left(\mathrm{GL}_{k c}(F) \backslash \mathrm{GL}_{k c}(\mathbb{A}), \eta\right)$ by right-translation and we denote the action by $g \cdot \varphi$, where $g \in \mathrm{GL}_{k c}(\mathbb{A})$.

Let $\rho_{0}$ be an irreducible subrepresentation of $L^{2}\left(\mathrm{GL}_{k c}(F) \backslash \mathrm{GL}_{k c}(\mathbb{A}), \eta\right)$ for some $\eta$ and $\rho=$ $|\operatorname{det}|^{r} \rho_{0}$ for some $r \in \mathbb{R}$. Assume $\rho$ is a $(k, c)$ representation. The space $W(\rho)$ of functions

$$
g \mapsto \Lambda(g \cdot \varphi),
$$

where $\varphi$ varies in the space of $\rho$, is called a global $(k, c)$ model of $\rho$.

Write $\rho=\otimes_{\nu}^{\prime} \rho_{\nu}$ as a restricted tensor product, with respect to a system $\left\{\xi_{\nu}^{0}\right\}_{\nu \notin S}$ of spherical vectors, where $S$ is a finite set of places of $F$ depending on $\rho$. For all $\nu \notin S, \rho_{\nu}$ is unramified 
and then the space $\operatorname{Hom}_{V_{\left(c^{k}\right)}\left(F_{\nu}\right)}\left(\rho_{\nu}, \psi_{\nu}\right)$ is one-dimensional. We fix $\Lambda_{\nu}^{0} \in \operatorname{Hom}_{V_{\left(c^{k}\right)}\left(F_{\nu}\right)}\left(\rho_{\nu}, \psi_{\nu}\right)$ at these places by requiring $\Lambda_{\nu}^{0}\left(\xi_{\nu}^{0}\right)=1$. We can further define

$$
\Lambda_{S} \in \operatorname{Hom}_{V_{\left(c^{k}\right)}\left(F_{S}\right)}\left(\rho_{S}, \psi_{S}\right),
$$

where the subscript $S$ denotes the finite product over the places of $S$ (e.g., $\rho_{S}=\otimes_{\nu \in S} \rho_{\nu}$ ), by

$$
\Lambda_{S}\left(\xi_{S}\right)=\Lambda\left(\xi_{S} \otimes_{\nu \notin S}^{\prime} \xi_{\nu}^{0}\right) .
$$

Then we have the following decomposition result.

Claim 4. Let $\varphi$ be a decomposable vector in the space of $\rho$, which we identify with the element $\xi_{S} \otimes_{\nu \notin S}^{\prime} \xi_{\nu}$ in $\otimes_{\nu}^{\prime} \rho_{\nu}$. Then for all $g \in \mathrm{GL}_{k c}(\mathbb{A})$,

$$
\Lambda(\rho(g) \varphi)=\Lambda_{S}\left(\rho_{S}\left(g_{S}\right) \xi_{S}\right) \prod_{\nu \notin S} \Lambda_{\nu}\left(\rho_{\nu}\left(g_{\nu}\right) \xi_{\nu}\right),
$$

where $\Lambda_{\nu}$ is a scalar multiple of $\Lambda_{\nu}^{0}$ for all $\nu \notin S$.

Proof. Similar to [Tak14, Proposition 3.14], which is an adaptation of the decomposition result when uniqueness holds everywhere (see [Sha74, § 4], [Bum97, Theorem 3.5.2]).

Let $F^{\prime}$ be a local field of characteristic 0 .

Definition 5. Let $\sigma$ be a smooth admissible finite length (complex) representation of $\mathrm{GL}_{k c}\left(F^{\prime}\right)$. We say that $\sigma$ is a $(k, c)$ representation if the following holds:

(1) For all unipotent orbits $\beta$ greater than or non-comparable with $\left(k^{c}\right), \operatorname{Hom}_{V(\beta)\left(F^{\prime}\right)}\left(\sigma, \psi_{\beta}^{\prime}\right)=0$, where $V(\beta)$ is the unipotent subgroup corresponding to $\beta$ and $\psi_{\beta}^{\prime}$ is any generic character of $V(\beta)$.

(2) The space $\operatorname{Hom}_{V_{\left(c^{k}\right)}\left(F^{\prime}\right)}(\sigma, \psi)$ (continuous morphisms over archimedean fields) is one-dimensional, where $\psi$ is defined by (2.3).

Any nonzero $\lambda \in \operatorname{Hom}_{V_{\left(c^{k}\right)}\left(F^{\prime}\right)}(\sigma, \psi)$ is called a $(k, c)$ functional on $\sigma$, and if we fix one such $\lambda$, the $(k, c)$ model $W(\sigma)$ is the space of functions $g \mapsto \lambda\left(\sigma(g) \xi^{\prime}\right)$ where $\xi^{\prime}$ varies in the space of $\sigma$ and $g \in \mathrm{GL}_{k c}\left(F^{\prime}\right)$. We mention that even if $\operatorname{Hom}_{V_{\left(c^{k}\right)}\left(F^{\prime}\right)}(\sigma, \psi)$ is not one-dimensional, we can still consider spaces of such functions, defined for each choice of $(k, c)$ functional, but they will typically depend on the choice of the functional, i.e., the model is not unique.

Example 6. $A(k, 1)$ representation is a representation of $\mathrm{GL}_{k}$ affording a unique Whittaker model.

Remark 7. If we do have local uniqueness everywhere, then we can decompose $W(\rho)=\otimes_{\nu}^{\prime} W\left(\rho_{\nu}\right)$ as a restricted tensor product (see the argument in $[$ Sha74, § 4]). This is the case, for example, when $c=1$ and the representation is globally generic.

Let $\varphi$ belong to the space of $\rho$. The Fourier coefficient $\Lambda(\varphi)$ enjoys an extra invariance property. Let $\mathrm{GL}_{c}^{\Delta}$ denote the image of $\mathrm{GL}_{c}$ inside $\mathrm{GL}_{k c}$ under the diagonal embedding $h \mapsto$ $h^{\Delta}=\operatorname{diag}(h, h, \ldots, h)$.

Claim 8. For all $h \in \mathrm{SL}_{c}(\mathbb{A}), \Lambda\left(h^{\Delta} \cdot \varphi\right)=\Lambda(\varphi)$.

Proof. The group $\mathrm{GL}_{c}^{\Delta}$ is the stabilizer of the character $\psi$ inside $M_{\left(c^{k}\right)}$. If we expand along any unipotent subgroup of $\mathrm{SL}_{c}(\mathbb{A})$, the nontrivial contribution to the expansion vanishes, because the nontrivial term of the expansion is associated with a unipotent orbit which is greater than or non-comparable with $\left(k^{c}\right)$, while the unipotent orbit attached to $\rho$ is $\left(k^{c}\right)$. See [FG16, Proposition 3] for details. 
We proceed to show that the generalized Speh representations are $(k, c)$ representations. Let $\tau$ denote an irreducible unitary cuspidal automorphic representation of $\mathrm{GL}_{k}(\mathbb{A}), \underline{s}=\left(s_{1}, \ldots, s_{c}\right) \in$ $\mathbb{C}^{c}$, and $E(g ; \xi, \underline{s})$ denote the Eisenstein series associated with the induced representation

$$
\operatorname{Ind}_{P_{\left(k^{c}\right)}(\mathbb{A})}^{\mathrm{GL}_{k c}(\mathbb{A})}\left(|\operatorname{det}|^{s_{1}} \tau \otimes \ldots \otimes|\operatorname{det}|^{s_{c}} \tau\right)
$$

where $\xi$ is a standard section. Let $\underline{s}_{0} \in \mathbb{C}^{c}$ be the point defined by

$$
s_{1}+\ldots+s_{c}=0 ; \quad s_{i}-s_{i+1}=1 ; \quad 1 \leq i \leq c-1 .
$$

The series has a simple multi-residue at $\underline{s}_{0}$,

$$
E_{\underline{s}_{0}}(g ; \xi)=\lim _{\underline{s} \rightarrow \underline{s}_{0}} \prod_{i=1}^{c-1}\left(s_{i}-s_{i+1}-1\right) M\left(w_{0}, \underline{s}\right) \xi(g, \underline{s}),
$$

where $M\left(w_{0}, \underline{s}\right)$ is the standard intertwining operator defined by (the meromorphic continuation of)

$$
M\left(w_{0}, \underline{s}\right) \xi(g, \underline{s})=\int_{V_{\left(k^{c}\right)}(\mathbb{A})} \xi\left(w_{0} u g, \underline{s}\right) d u, \quad w_{0}=\left({ }_{I_{c}}^{I_{c}}{ }^{I_{c}}\right) .
$$

The automorphic representation $\mathcal{E}_{\tau}$ of $\mathrm{GL}_{k c}(\mathbb{A})$ generated by all the residue functions $E_{\underline{s}_{0}}(\cdot ; \xi)$ lies in the discrete spectrum of the space $L^{2}\left(\mathrm{GL}_{k c}(F) \backslash \mathrm{GL}_{k c}(\mathbb{A}), \eta_{\tau}^{c}\right)$, where $\eta_{\tau}$ is the central character of $\tau$ ([Lan76, MW95]), and is irreducible ([MW89]).

Furthermore, write $\tau=\otimes_{\nu}^{\prime} \tau_{\nu}$. At all places, $\tau_{\nu}$ is irreducible unitary and generic, and at almost all places $\tau_{\nu}$ is unramified and can be written in the form

$$
\operatorname{Ind}_{B_{\mathrm{GL}_{k}}\left(F_{\nu}\right)}^{\mathrm{GL}_{k}\left(F_{\nu}\right)}\left(\chi_{1} \otimes \ldots \otimes \chi_{k}\right),
$$

where $\chi_{1}, \ldots, \chi_{k}$ are unramified quasi-characters of $F_{\nu}^{*}$. In this case we also denote

$$
\sigma_{k, c}=\operatorname{Ind}_{P_{\left(c^{k}\right)}\left(F_{\nu}\right)}^{\mathrm{GL}_{k c}\left(F_{\nu}\right)}\left(\chi_{1} \otimes \ldots \otimes \chi_{k}\right),
$$

where each $\chi_{i}$ is pulled back to a character of $\mathrm{GL}_{c}\left(F_{\nu}\right)$ using det. Observe that since $\tau_{\nu}$ is unitary, by [JS83, Corollary 2.5] (applied to $\tau_{\nu}$ and $\tau_{\nu}^{\vee}$ ), $q_{\nu}^{-1 / 2}<\left|\chi_{i}\right|<q_{\nu}^{1 / 2}$ for all $i$, where $q_{\nu}$ is the residue cardinality of $F_{\nu}$. Thus the segments corresponding to $\chi_{i} \circ$ det and $\chi_{j} \circ$ det, for all $i \neq j$, are not linked, using the terminology of Zelevinsky [Zel80, $\S 3, \S 4]$, and then $\sigma_{k, c}$ is irreducible [Zel80, Theorem 4.2].

To extend the applicability of some of our local arguments, we define $\sigma_{k, c}$ in the same way for arbitrary unramified quasi-characters $\chi_{i}$, i.e., not necessarily the inducing data of an irreducible unitary generic representation of $\mathrm{GL}_{k}\left(F_{\nu}\right)$. Then $\sigma_{k, c}$ may be reducible.

Claim 9. Assume $\tau_{\nu}$ is given by (2.5) and let $\sigma_{k, c}$ be given by (2.6). Then $\left(\mathcal{E}_{\tau}\right)_{\nu}=\sigma_{k, c}$.

Proof. Since $\left(\mathcal{E}_{\tau}\right)_{\nu}$ is irreducible $([\mathrm{MW} 89])$, by construction it is the unique irreducible unramified quotient of

$$
\operatorname{Ind}_{P_{\left(k^{c}\right)}\left(F_{\nu}\right)}^{\mathrm{GL}_{k c}\left(F_{\nu}\right)}\left(\left(\tau_{\nu} \otimes \ldots \otimes \tau_{\nu}\right) \delta_{P_{\left(k^{c}\right)}}^{1 /(2 k)}\right) .
$$

Permuting the inducing characters of $\tau_{\nu}$ in the full induced representation (2.7), we reach

$$
\operatorname{Ind}_{B_{\mathrm{GL}_{k c}}\left(F_{\nu}\right)}^{\mathrm{GL}_{k c}\left(F_{\nu}\right)}\left(\chi_{1} \delta_{B_{\mathrm{GL}_{c}}}^{1 / 2} \otimes \ldots \otimes \chi_{k} \delta_{B_{\mathrm{GL}_{c}}}^{1 / 2}\right) .
$$

Here $\chi_{i} \delta_{B_{\mathrm{GL}}}^{1 / 2}$ is regarded as a representation of $T_{\mathrm{GL}_{c}}$. By Bernstein and Zelevinsky [BZ77, Theorem 2.9], the constituents of (2.7) and (2.8) are isomorphic (including multiplicities). 
Therefore the unique irreducible unramified quotient of $(2.7)$, which is $\left(\mathcal{E}_{\tau}\right)_{\nu}$, is the unique irreducible unramified constituent of (2.8).

Since the trivial representation is the unique irreducible unramified quotient of $\operatorname{Ind}_{B_{\mathrm{GL}_{c}}}^{\mathrm{GL}_{c}}\left(\delta_{B_{\mathrm{GL}_{c}}}^{1 / 2}\right)$, $\sigma_{k, c}$ is an unramified quotient of (2.8). Therefore $\left(\mathcal{E}_{\tau}\right)_{\nu}$ is already a constituent of $\sigma_{k, c}$, which is irreducible because $\tau_{\nu}$ is unitary, as explained above.

Claim 10. The representation $\sigma_{k, c}$ with arbitrary unramified quasi-characters $\chi_{i}$ is $(k, c)$.

Proof. We proceed with local notation and omit references to the field. For any composition $\lambda$ of $k c$, let $\psi_{\lambda}$ be the character of $N_{\mathrm{GL}_{k c}}$ which restricts to $\psi$ on the simple root subgroups of $M_{\lambda}$ and acts trivially otherwise. Extend the partial order on partitions to compositions by comparing their underlying partitions. According to [Cai18, Proposition 5.5] (see also [MW87, GGS17]), to deduce the vanishing property it is enough to prove that for any $\lambda$ which is greater than or non-comparable with $\left(k^{c}\right)$, the twisted Jacquet module $J_{N_{\mathrm{GL}_{k c}}, \psi_{\lambda}}\left(\sigma_{k, c}\right)$ vanishes.

Assuming $\lambda_{i}>k$ for some $i$, we prove $J_{N_{\mathrm{GL}_{k c}}, \psi_{\lambda}}\left(\sigma_{k, c}\right)=0$. We argue by induction on $k$. When $k=1$ this is trivial because $\sigma_{1, c}$ is a character of $\mathrm{GL}_{c}$. Since $\sigma_{k, c}=\sigma_{k-1, c} \times \sigma_{1, c}$, where $\times$ is the parabolic induction functor (see [BZ77]), by [BZ77, 4.14] $J_{N_{\mathrm{GL}_{k c}} \psi_{\lambda}}\left(\sigma_{k, c}\right)$ is glued from the representations

$$
J_{N_{\mathrm{GL}_{(k-1) c}}, \psi_{\lambda^{\prime}}}\left(\sigma_{k-1, c}\right) \times J_{N_{\mathrm{GL}_{c}}, \psi_{\lambda^{\prime \prime}}}\left(\sigma_{1, c}\right),
$$

where $\lambda^{\prime}$ and $\lambda^{\prime \prime}$ vary over the compositions of $(k-1) c$ and $c$ (resp.) such that $\lambda_{i}=\lambda_{i}^{\prime}+\lambda_{i}^{\prime \prime}$ for all $i$. If $\lambda_{i}>k$ for some $i$, then either $\lambda_{i}^{\prime}>(k-1)$ or $\lambda_{i}^{\prime \prime}>1$, whence by the induction hypothesis all the representations vanish. Thus $J_{N_{\mathrm{GL}}, \psi_{\lambda}}\left(\sigma_{k, c}\right)=0$.

It remains to show $\operatorname{dim} J_{V_{\left(c^{k}\right)}, \psi}\left(\sigma_{k, c}\right)=1$. By [Cai18, Proposition 5.5], $\operatorname{dim} J_{V_{\left(c^{k}\right)}, \psi}\left(\sigma_{k, c}\right)=$ $\operatorname{dim} J_{N_{\mathrm{GL}_{k c}} \psi_{\left(k^{c}\right)}}\left(\sigma_{k, c}\right)$, so that we can prove $\operatorname{dim} J_{N_{\mathrm{GL}_{k c}}, \psi_{\left(k^{c}\right)}}\left(\sigma_{k, c}\right)=1$, using induction on $k$. This is clear for $k=1$. Now looking at the filtration above with $\lambda=\left(k^{c}\right)$, the contribution is nontrivial if and only if $\lambda^{\prime}=\left((k-1)^{c}\right)$ and $\lambda^{\prime \prime}=\left(1^{c}\right)$. Applying the induction hypothesis, $\operatorname{dim} J_{N_{\mathrm{GL}}(k-1),}, \psi_{\lambda^{\prime}}\left(\sigma_{k-1, c}\right)=1$, thus $J_{N_{\mathrm{GL}_{k c}} \psi_{\lambda}}\left(\sigma_{k, c}\right)$ is one-dimensional.

Remark 11. Note that this result holds without any assumption on $\sigma_{k, c}$.

Remark 12. Fourier coefficients corresponding to $N_{\mathrm{GL}_{k c}}$ and $\psi_{\lambda}$ are called semi-Whittaker coefficients. They are intimately related to Fourier coefficients associated with unipotent orbits, both locally and globally, see [AGS15, Cai18, GGS17, GGS, MW87] (an archimedean analog of Claim 10 appeared in [AGS15]).

Theorem 13. Let $\tau$ be an irreducible cuspidal automorphic representation of $\mathrm{GL}_{k}(\mathbb{A})$. The representation $\mathcal{E}_{\tau}$ is a $(k, c)$ representation.

Proof. Since $\tau=|\operatorname{det}|^{d} \tau_{0}$ for some $d \in \mathbb{R}$ and a similar representation $\tau_{0}$ which is also unitary, we can already assume $\tau$ is unitary. The global condition of Definition 3 was proved in [Gin03, Proposition 5.3] (see also [JL13]). The local condition now follows immediately from Claims 9 and 10.

Remark 14. It is important to note that the representations $\sigma_{k, c}$ are special, in the sense that they admit a unique $(k, c)$ functional. We do not expect an arbitrary irreducible representation $\sigma$ of $\mathrm{GL}_{k c}$ to enjoy this property. In fact for $c>1$, the dimension of $J_{V_{\left(c^{k}\right)}, \psi}(\sigma)$ can be infinite. We also mention that for $k=2$, the character $(2.3)$ is the Shalika character, and again $J_{V_{\left(c^{2}\right)}, \psi}(\sigma)$ can be infinite dimensional: to obtain uniqueness results we require the additional invariance property with respect to the reductive part of the stabilizer - the diagonal embedding of $\mathrm{GL}_{c}$ in 
$\mathrm{GL}_{2 c}$. Nonetheless, for specific representations (similar to $\sigma_{2, c}$ ) invariance with respect to the reductive part is automatic (see e.g., [BB06]).

2.3. Unfolding of the global integral for symplectic groups. In this section we complete the proof of Theorem 1 for the symplectic group. Let $G=\mathrm{Sp}_{2 n}$ and recall that $c=2 n, H=\mathrm{Sp}_{4 k n}$ and $Q=M \ltimes U$ where

$$
M=\mathrm{GL}_{2 n} \times \ldots \times \mathrm{GL}_{2 n} \times \mathrm{Sp}_{4 n} .
$$

Here $\mathrm{GL}_{2 n}$ appears $k-1$ times. Identify the quotient $U /[U, U]$ with

$$
\mathrm{Mat}_{2 n} \oplus \ldots \oplus \mathrm{Mat}_{2 n} \oplus \mathrm{Mat}_{2 n \times 4 n},
$$

where $\operatorname{Mat}_{2 n}$ appears $k-2$ times. For $Y \in \operatorname{Mat}_{2 n \times 4 n}$ write

$$
Y=\left(\begin{array}{ccc}
Y_{1} & Z_{1} & Y_{2} \\
Y_{3} & Z_{2} & Y_{4}
\end{array}\right), \quad Y_{i} \in \operatorname{Mat}_{n}, Z_{j} \in \operatorname{Mat}_{n \times 2 n} .
$$

Let $\psi_{U}$ be the pullback to $U$ of the character of $U /[U, U]$ given by

$$
\left(X_{1}, \ldots, X_{k-2}, Y\right) \mapsto \psi\left(\operatorname{tr}\left(X_{1}+\cdots+X_{k-2}+Y_{1}+Y_{4}\right)\right) .
$$

The corresponding Fourier coefficient given by $U$ and $\psi_{U}$ is associated with the unipotent orbit $\left((2 k-1)^{2 n} 1^{2 n}\right)$. The embedding of $G \times G$ in $H$ is given by

$$
\left(g_{1}, g_{2}\right) \mapsto \operatorname{diag}\left(g_{1}, \ldots, g_{1},\left(\begin{array}{lll}
g_{1,1} & & g_{1,2} \\
& g_{2} & \\
g_{1,3} & & g_{1,4}
\end{array}\right), g_{1}^{*}, \ldots, g_{1}^{*}\right),
$$

where $g_{1}=\left(\begin{array}{ll}g_{1,1} & g_{1,2} \\ g_{1,3} & g_{1,4}\end{array}\right), g_{1, i} \in \mathrm{Mat}_{n}$ and $g_{1}^{*}=J_{2 n}{ }^{t} g_{1}^{-1} J_{2 n}$ appears $k-1$ times. Note that the middle $4 n \times 4 n$ block is the standard embedding of $G \times G$ in the middle $\operatorname{Sp}_{4 n}$ block of $M$. The involution $\iota$ is defined by ${ }^{\iota} g=\iota g \iota^{-1}$ with

$$
\iota=\left({ }_{I_{n}}^{I_{n}}\right)
$$

We have to show that for $\operatorname{Re}(s) \gg 0$,

$$
\begin{aligned}
Z\left(s, \varphi_{1}, \varphi_{2}, f\right) & =\int_{G(F) \times G(F) \backslash G(\mathbb{A}) \times G(\mathbb{A})} \int_{U(F) \backslash U(\mathbb{A})} \varphi_{1}\left(g_{1}\right) \overline{\varphi_{2}\left({ }^{\iota} g_{2}\right)} E\left(u\left(g_{1}, g_{2}\right) ; f, s\right) \psi_{U}(u) d u d g_{1} d g_{2} \\
& =\int_{G(\mathbb{A})} \int_{U_{0}(\mathbb{A})}\left\langle\varphi_{1}, \pi(g) \varphi_{2}\right\rangle f_{W\left(\mathcal{E}_{\tau}\right)}\left(\delta u_{0}\left(1,{ }^{\iota} g\right), s\right) \psi_{U}\left(u_{0}\right) d u_{0} d g .
\end{aligned}
$$

(The right hand side is (2.1).) The element $\delta$ is given in (2.18).

Recall that $P=M_{P} \ltimes U_{P}$ is the standard maximal parabolic subgroup of $H$ with $M_{P} \cong \mathrm{GL}_{2 k n}$, and let $L=(G \times G) U$ denote the subgroup of $Q$ embedded in $H$ as described above. In general for $h, h^{\prime} \in H$ and $H^{\prime}<H$, put

$$
{ }^{h} h^{\prime}=h h^{\prime} h^{-1}, \quad{ }^{h} H^{\prime}=\left\{{ }^{h} h^{\prime}: h^{\prime} \in H^{\prime}\right\} .
$$

Unfolding the Eisenstein series in $Z\left(s, \varphi_{1}, \varphi_{2}, f\right)$, the integral becomes

$$
\sum_{\gamma \in P(F) \backslash H(F) / L(F)} \mathrm{I}(\gamma)
$$

where

$$
\mathrm{I}(\gamma)=\int_{L_{\gamma}(F) \backslash L(\mathbb{A})} \varphi_{1}\left(g_{1}\right) \overline{\varphi_{2}\left({ }^{\iota} g_{2}\right)} f\left(\gamma u\left(g_{1}, g_{2}\right), s\right) \psi_{U}(u) d u d g_{1} d g_{2}
$$


Here $L_{\gamma}=\gamma^{-1} P \cap L$. We show that there is a unique representative $\gamma$ such that $\mathrm{I}(\gamma)$ is equal to integral (2.1), and that for all other representatives $\mathrm{I}(\gamma)=0$. The representative contributing to the sum corresponds to the open orbit.

In general, there are three ways to show $\mathrm{I}(\gamma)=0$ (and we use all three). The first is using the character $\psi_{U}$. Specifically, if there is a unipotent subgroup $U^{\prime}$ of $U$ on which $\psi_{U}$ is nontrivial and $\gamma U^{\prime}<U_{P}$, the integral $\mathrm{I}(\gamma)$ vanishes because the integral of $\psi_{U}$ on $U^{\prime}(F) \backslash U^{\prime}(\mathbb{A})$ is zero. The second option is to use the cuspidality of $\pi_{i}$ : if $L_{\gamma}$ contains a unipotent radical $V$ of a parabolic subgroup of one of the copies of $G$, and the suitable integral over $f$ is invariant under $V(\mathbb{A})$, then $\mathrm{I}(\gamma)$ vanishes because $\pi_{i}$ is cuspidal. The third alternative is to use the smallness of $\mathcal{E}_{\tau}$, which is the $(k, 2 n)$ representation appearing in the inducing data of the Eisenstein series, and is attached to the unipotent orbit $\left(k^{2 n}\right)$ of $\mathrm{GL}_{2 k n}$. Thus, if we obtain as an inner integration a Fourier coefficient attached to a unipotent orbit which is greater than or non-comparable with $\left(k^{2 n}\right)$, then we get zero contribution from this representative.

We begin with a parametrization of the representatives $\gamma$ of $P(F) \backslash H(F) / L(F)$. Let $N_{H}$ be the unipotent radical of $B_{H}$. By the Bruhat decomposition the double cosets $P \backslash H / B_{H}=$ $P \backslash H / N_{H}$ can be represented using Weyl elements, and since $N_{H}<Q=M U$, every representative $\gamma$ can be written in the form

$$
\gamma=w u
$$

for a Weyl element $w$ of $H$ and $u \in M \cap N_{H}$. In the following, we will gradually reduce the number of possible representatives contributing to (2.12), until we remain with only one, which we will denote by $\delta$. Hence $Z\left(s, \varphi_{1}, \varphi_{2}, f\right)$ is equal to $\mathrm{I}(\delta)$, which will then be slightly modified to produce integral (2.1).

Our main tool for reducing the number of representatives is the following claim. Its proof, along with the proofs of several subsequent statements, is deferred until later in this section.

Lemma 15. If $\gamma=w u$ and there is a one-parameter subgroup $U^{\prime}$ of $U$ such that $\left.\psi_{U}\right|_{U^{\prime}} \neq 1$ and ${ }^{w} U^{\prime}<U_{P}$, then $\mathrm{I}(\gamma)=0$.

Using the action of $\mathrm{GL}_{2 k n}$ on the left and $\left(I_{2 n}, G\right)$ on the right, we may assume

$$
w=\left(\begin{array}{ccccc}
\mu_{1} & & & & \mu_{2} \\
& \epsilon_{1} & & \epsilon_{2} & \\
& & I_{2 n} & & \\
& \epsilon_{3} & & \epsilon_{4} & \\
\mu_{3} & & & & \mu_{4},
\end{array}\right),
$$

where $\mu_{i} \in \operatorname{Mat}_{(k-1) 2 n} ; \epsilon_{i} \in \operatorname{Mat}_{n} ; \mu_{1}, \epsilon_{1}, \epsilon_{4}$ and $\mu_{4}$ are diagonal matrices whose entries are zeros and ones; $\mu_{2}, \epsilon_{2}, \epsilon_{3}$ and $\mu_{3}$ are matrices whose nonzero entries are on the anti-diagonal; the nonzero entries of $\mu_{2}$ and $\epsilon_{2}$ are ones; the nonzero entries of $\epsilon_{3}$ and $\mu_{3}$ are -1 . Since $w \in H$ (and is a Weyl element), it is completely determined by $\mu_{1}$ and $\epsilon_{1}$. Further write $\mu_{1}=\operatorname{diag}\left(\mu_{1,1}, \mu_{1,2}, \ldots, \mu_{1, k-1}\right)$ where $\mu_{1, i} \in \operatorname{Mat}_{2 n}$. We shall denote the $(l, l)$-th entry of $\mu_{1, i}$ by $\mu_{1, i}(l)$. Similarly, $\epsilon_{1}(l)$ is the $(l, l)$-th coordinate of $\epsilon_{1}$.

Set $u=u^{1} u^{2}$, with an upper triangular matrix $u^{2} \in \operatorname{Sp}_{4 n}\left(\operatorname{Sp}_{4 n}<M\right)$. Using $\left(G, I_{2 n}\right)$ we may assume

$$
u^{2}=\left(\begin{array}{ccccc}
I_{(k-1) 2 n} & & & & \\
& I_{n} & T & & \\
& & I_{2 n} & T^{\prime} & \\
& & & I_{n} & \\
& & & & I_{(k-1) 2 n}
\end{array}\right), \quad T=\left(\begin{array}{ll}
T_{1} & 0
\end{array}\right)
$$


where $T^{\prime}$ is defined uniquely by $T$ and the definition of $H$, and $T_{1} \in \operatorname{Mat}_{n}$. Put $u^{1}=\left(u_{1}, u_{2}, \ldots, u_{k-1}\right)$, for upper triangular matrices $u_{i} \in \mathrm{GL}_{2 n}$, and regard $u^{1}$ as an element in the product of $k-1$ factors of $\mathrm{GL}_{2 n}$ in $M$.

For any $h, h^{\prime} \in H$, write $h \sim h^{\prime}$ if $P h L=P h^{\prime} L$.

Claim 16. If $u^{1}$ is nontrivial, either $\mathrm{I}(\gamma)=0$ or $\gamma \sim w u^{2}$.

Fix $\gamma=w u^{2}$ as in (2.13) and (2.14). For $1 \leq i \leq n-1$, let $v_{i}$ denote the simple reflections in the Weyl group of $G$, which are contained inside the standard maximal parabolic subgroup of $G$ whose Levi part is $\mathrm{GL}_{n}$ (the Siegel parabolic subgroup). Using the reflections

$$
\mathrm{e}\left(v_{i}\right)=\left(v_{i}, I_{2 n}\right) \in H,
$$

we may assume $\epsilon_{1}=\operatorname{diag}\left(I_{j}, 0_{n-j}\right)$, where $0_{n-j} \in \mathrm{Mat}_{n-j}$ is the zero matrix and $0 \leq j \leq n$. This implies $\epsilon_{4}=\operatorname{diag}\left(0_{n-j}, I_{j}\right)$, whence

$$
u^{2}=\left(\begin{array}{cccccc}
I_{(k-1) 2 n+j} & & & & & \\
& I_{n-j} & T & & & \\
& & I_{n} & & & \\
& & & I_{n} & T^{\prime} & \\
& & & & I_{n-j} & \\
& & & & & I_{(k-1) 2 n+j}
\end{array}\right) .
$$

Claim 17. Assume that $u^{2}$ takes the form (2.16). Then $\mathrm{I}(\gamma)=0$ unless there is some $0 \leq j<n$ such that $\mu_{1, l}=\operatorname{diag}\left(I_{j_{l}}, 0_{2 n-j_{l}}\right)$ with $0 \leq j_{l} \leq j$ for all $1 \leq l \leq k-1$.

Claim 18. If $j>0, \mathrm{I}(\gamma)=0$.

It remains to consider $j=0$. Thus $j_{l}=0$ for all $l$. This already implies $w=\left(\begin{array}{c}I_{2 k n} \\ -I_{2 k n}\end{array}\right)$. Multiplying on the right by $\left(I_{2 n},\left({ }_{-I_{n}}{ }^{I_{n}}\right)\right)$ (this shifts the block $T$ in $(2.16)$ and $w\left(I_{2 n},\left({ }_{-I_{n}} I_{n}\right)\right) \epsilon$ $P)$, we need to consider all representatives with

$$
u^{2}=u^{2}[T]=\left(\begin{array}{cccccc}
I_{(k-1) 2 n} & & & & & \\
& I_{n} & & T & & \\
& & I_{n} & & T^{\prime} & \\
& & & I_{n} & & \\
& & & & I_{n} & \\
& & & & & I_{(k-1) 2 n}
\end{array}\right) .
$$

The group $\mathrm{GL}_{n} \times \mathrm{GL}_{n}$, embedded inside $G \times G$ as the group of matrices of the form

$$
[A, B]=\left(\left({ }_{A^{*}}{ }^{*}\right),\left({ }^{B}{ }_{B^{*}}\right)\right) \quad\left(X^{*}=J_{n}{ }^{t} X^{-1} J_{n}\right),
$$

acts on all matrices $(2.17)$ by $[A, B] \cdot u^{2}[T]=u^{2}\left[A T\left(J_{n}{ }^{t} B J_{n}\right)\right]$. Hence, a set of representatives for this action can be taken to be any $n+1$ matrices whose ranks are $0,1, \ldots, n$, e.g., the matrices $\left(I_{l}{ }^{0}\right)$, where $0 \leq l \leq n$.

Claim 19. If $T=\left({ }_{I_{l}}{ }^{0}\right)$ with $0 \leq l<n, \mathrm{I}(\gamma)=0$.

Finally denote the remaining representative by $\delta$,

$$
\delta=\left(\begin{array}{cc} 
& I_{2 k n} \\
-I_{2 k n} &
\end{array}\right)\left(\begin{array}{llll}
I_{(k-1) 2 n} & & & \\
& I_{2 n} & I_{2 n} & \\
& & I_{2 n} & \\
& & & I_{(k-1) 2 n}
\end{array}\right) .
$$


The group $L_{\delta}$ is described as follows. First, inside $G \times G$ we obtain the group $G$ embedded as $g \mapsto\left(g,{ }^{\imath} g\right)$. In $U$, we obtain the subgroup $V$ of all matrices of the form

$$
\left(\begin{array}{cccc}
u_{1} & & & \\
& I_{2 n} & & \\
& & I_{2 n} & \\
& & & u_{1}^{*}
\end{array}\right)\left(\begin{array}{cccc}
I_{(k-1) 2 n} & u_{2} & -u_{2} & 0 \\
& I_{2 n} & & -u_{2}^{\prime} \\
& & I_{2 n} & -u_{2}^{\prime} \\
& & & I_{(k-1) 2 n}
\end{array}\right) \quad\left(u_{2}^{\prime}=J_{2 n}{ }^{t} u_{2} J_{(k-1) 2 n}\right) .
$$

Since all summands but $\mathrm{I}(\gamma)=\mathrm{I}(\delta)$ vanish,

$$
Z\left(s, \varphi_{1}, \varphi_{2}, f\right)=\mathrm{I}(\delta)=\int_{L_{\delta}(F) \backslash L(\mathbb{A})} \varphi_{1}\left(g_{1}\right) \overline{\varphi_{2}\left({ }^{\circ} g_{2}\right)} f\left(\delta u\left(g_{1}, g_{2}\right), s\right) \psi_{U}(u) d u d g_{1} d g_{2} .
$$

We factor the integration through $L_{\delta}(\mathbb{A})$. The quotient $L_{\delta} \backslash L=\left(I_{2 n}, G\right) \ltimes U_{0}$, where we recall that $U_{0}=U \cap U_{P}$. Therefore (2.19) becomes

$$
\int_{G(\mathbb{A})} \int_{U_{0}(\mathbb{A})} \int_{G(F) \backslash G(\mathbb{A})} \int_{V(F) \backslash V(\mathbb{A})} \varphi_{1}\left(g_{1}\right) \overline{\varphi_{2}\left({ }^{\iota}\left({ }^{\iota} g_{1} g_{2}\right)\right)} f\left(\delta v u_{0}\left(g_{1},{ }^{\iota} g_{1}\right)\left(1, g_{2}\right), s\right) \psi_{U}\left(v u_{0}\right) d v d g_{1} d u_{0} d g_{2} .
$$

Conjugating $V$ across $\delta$, we obtain the subgroup $V_{\left((2 n)^{k}\right)}$ of $\mathrm{GL}_{2 k n}$, where $V_{\left((2 n)^{k}\right)}$ is the unipotent subgroup defined in $\S 2.2$, and after adjusting $\psi_{U}(v)$ we obtain the character $(2.3)$, so that we can write the integral in the form

$$
\int_{G(\mathbb{A})} \int_{U_{0}(\mathbb{A})} \int_{G(F) \backslash G(\mathbb{A})} \varphi_{1}\left(g_{1}\right) \overline{\varphi_{2}\left(g_{1}{ }^{\iota} g_{2}\right)} f_{W\left(\mathcal{E}_{\tau}\right)}\left(\delta u_{0}\left(g_{1},{ }^{\imath} g_{1}\right)\left(1, g_{2}\right), s\right) \psi_{U}\left(u_{0}\right) d g_{1} d u_{0} d g_{2} .
$$

We remind the reader that

$$
f_{W\left(\mathcal{E}_{\tau}\right)}(h, s)=\int_{V_{\left((2 n)^{k}\right)}(F) \backslash V_{\left((2 n)^{k}\right)}(\mathbb{A})} f(v h, s) \psi^{-1}(v) d v,
$$

where $\psi$ is defined by $(2.3)$. By Claim 8 , the $(k, 2 n)$ functional is invariant under $\mathrm{SL}_{2 n}^{\Delta}(\mathbb{A})$. Since ${ }^{\delta}\left(g_{1},{ }^{\iota} g_{1}\right)=\operatorname{diag}\left(g_{1}, \ldots, g_{1}\right) \in \mathrm{SL}_{2 n}^{\Delta}(\mathbb{A})$, the integral over $G(F) \backslash G(\mathbb{A})$ produces the inner product $\left\langle\varphi_{1}, \pi\left({ }^{\prime} g_{2}\right) \varphi_{2}\right\rangle$. Changing $g_{2} \mapsto{ }^{\prime} g_{2}$, we reach

$$
\left.\int_{G(\mathbb{A})} \int_{U_{0}(\mathbb{A})}\left\langle\varphi_{1}, \pi\left(g_{2}\right) \varphi_{2}\right\rangle f_{W\left(\mathcal{E}_{\tau}\right)}\left(\delta u_{0}\left(1,{ }^{\imath} g_{2}\right), s\right)\right) \psi_{U}\left(u_{0}\right) d u_{0} d g_{2} .
$$

This is the integral (2.1). In particular $\mathrm{I}(\delta)=0$ whence $Z\left(s, \varphi_{1}, \varphi_{2}, f\right)$ itself vanishes, unless $\pi_{1}=\pi_{2}=\pi$. The proof of the theorem is complete.

Remark 20. The only differences in the construction for $G=\mathrm{SO}_{2 n}$ are that $M=\mathrm{GL}_{2 n} \times \ldots \times$ $\mathrm{GL}_{2 n} \times \mathrm{SO}_{4 n}$ and $\iota=\left({ }_{-I_{n}}^{I_{n}}\right)$. The remaining parameters are similar: the character $\psi_{U}$ is still given by (2.9), the embedding $\left(g_{1}, g_{2}\right)$ is the same, $U_{0}=U \cap U_{P}, \mathcal{E}_{\tau}$ (still) corresponds to the unipotent orbit $\left(k^{2 n}\right)$, and $\delta=\left(\begin{array}{cc}I_{2 k n} \\ I_{2 k n}\end{array}\right) \operatorname{diag}\left(I_{(k-1) 2 n},\left(\begin{array}{cc}I_{2 n} & A \\ I_{2 n}\end{array}\right), I_{(k-1) 2 n}\right)$ with $A=\left(\begin{array}{cc}-I_{n} & \\ & I_{n}\end{array}\right)$.

Proof of Lemma 15. The result clearly follows if ${ }^{\gamma} U^{\prime}<U_{P}$ : indeed by definition, $U^{\prime}<\gamma^{-1} P \cap U<$ $L_{\gamma}$, so that we can factor the integral $\mathrm{I}(\gamma)$ through $U^{\prime}(F) \backslash U^{\prime}(\mathbb{A})=F \backslash \mathbb{A}\left(U^{\prime}\right.$ is a one-parameter subgroup), then the left invariance properties of $f$ yield an inner integration $\int_{F \backslash \mathbb{A}} \psi(u) d u$, which vanishes.

Assume that $U^{\prime}$ exists with the stated properties. We will show that ${ }^{w} U^{\prime}<U_{P}$ implies the existence of another one-parameter subgroup $U^{\prime \prime}$ of $U$ such that ${ }^{\gamma} U^{\prime \prime}<U_{P}$ and $\left.\psi_{U}\right|_{U^{\prime \prime}} \neq 1$, whence $\mathrm{I}(\gamma)=0$. 
For $1 \leq i<j \leq 4 k n$, let $x_{i, j}$ denote the one-parameter unipotent subgroup along the positive root $(i, j)$ of $H$. Put

$$
x_{i}= \begin{cases}x_{i, 2 n+i} & 1 \leq i \leq(k-1) 2 n-n, \\ x_{i, 4 n+i} & (k-1) 2 n-n+1 \leq i \leq(k-1) 2 n .\end{cases}
$$

Then $\left.\psi_{U}\right|_{x_{i}} \neq 1$ for all $i$, and $\psi_{U}$ is trivial on any other subgroup $x_{i, j}$ contained in $U$. Our assumption is thus ${ }^{w} x_{i} \in U_{P}$ for some $i$. Since $u \in M(\gamma=w u), U^{\prime \prime}=u^{-1} x_{i}<U$, and because $u$ is unipotent, $\psi_{U}\left(x_{i}(a)\right)=\psi_{U}\left({ }^{u^{-1}} x_{i}(a)\right)$ for $a \in \mathbb{A}$. Hence $U^{\prime \prime}$ has the required properties.

Proof of Claim 16. The proof is by induction on $\left(l, i_{1}, i_{2}\right)$ where $1 \leq l \leq k-1$ and $1 \leq i_{1}<i_{2} \leq 2 n$. Assume that the $\left(i_{1}, i_{2}\right)$-th entry of $u_{l}$ is nonzero, where $l$ is minimal such that for all $\left(i_{1}^{\prime}, i_{2}^{\prime}\right)$ with either $i_{1}^{\prime}<i_{2}^{\prime}<i_{2}$ or $i_{1}<i_{1}^{\prime}<i_{2}^{\prime}=i_{2}$, and all $l \leq l^{\prime} \leq k-1$, the $\left(i_{1}^{\prime}, i_{2}^{\prime}\right)$-th entry of $u_{l^{\prime}}$ is zero. For instance if the $(1,2)$-th coordinate of $u_{1}$ is nonzero, we set $l=1$ and $\left(i_{1}, i_{2}\right)=(1,2)$.

In general, if we can write $u^{1}=u^{\prime} v^{1}$ where ${ }^{w} u^{\prime} \in P$ and $v^{1}$ is of the same form as $u^{1}$, that is, an element in the product of $k-1$ factors of $\mathrm{GL}_{2 n}$ in $M$, then $h=w u^{1} u^{2} \sim w v^{1} u^{2}$. Hence if $\left(l, i_{1}, i_{2}\right)$ are given, it is implicitly assumed we cannot write $u^{1}=u^{\prime} v^{1}$ where the $\left(i_{1}, i_{2}\right)$-th coordinate of $v^{1}$ is zero.

All $1 \leq l \leq k-1$ are handled in the same manner, and for simplicity we assume $l=1$. Denote by $e_{i_{1}, i_{2}} \in$ Mat $_{2 n}$ the matrix whose $\left(i_{1}, i_{2}\right)$-th entry is one and whose other entries are all zero, and for $a \in \mathbb{A}$ put $y_{i_{1}, i_{2}}(a)=I_{2 n}+a e_{i_{1}, i_{2}}$. Then

$$
u^{1}=\left(y_{i_{1}, i_{2}}\left(t_{1}\right) u_{1}^{\prime}, y_{i_{1}, i_{2}}\left(t_{2}\right) u_{2}^{\prime}, \ldots, y_{i_{1}, i_{2}}\left(t_{k-1}\right) u_{k-1}^{\prime}\right)
$$

with $t_{1} \neq 0$. The matrices $u_{l}^{\prime} \in \operatorname{Mat}_{2 n}$ are upper triangular unipotent matrices whose $\left(j_{1}, j_{2}\right)$-th entries are zero for all $i_{1} \leq j_{1}$ and $j_{2} \leq i_{2}$. We show that either $\mathrm{I}(\gamma)=0$ or

$$
w u \sim w v^{1} v^{2},
$$

where $v^{1}=\left(u_{1}^{\prime}, u_{2}^{\prime}, \ldots, u_{k-1}^{\prime}\right)$ and $v^{2}$ takes the form $(2.14)$.

There are three cases to consider. Either $i_{1}<i_{2} \leq n, i_{1} \leq n<i_{2}$, or $n<i_{1}<i_{2}$. We will present the details in the second case; the other two cases are treated similarly. Assume

$$
1 \leq i_{1} \leq n, \quad i_{2}=n+j, \quad 1 \leq j \leq n .
$$

There are initially 4 possibilities for the values of $\mu_{1,1}\left(i_{1}\right)$ and $\mu_{1,1}(n+j)$. Matrix multiplication implies

$$
\mu_{1,1}\left(i_{1}\right)=0, \quad \mu_{1,1}(n+j)=1
$$

since otherwise ${ }^{w}\left(y_{i_{1}, i_{2}}\left(t_{1}\right), 1, \ldots, 1\right) \in P$, contradicting our assumption.

Now we show

$$
\forall 2 \leq l \leq k-1, \quad \mu_{1, l}(n+j)=1, \quad \text { and } \quad \epsilon_{4}(j)=0 .
$$

Here $\epsilon_{4}(j)$ is the $(j, j)$-th coordinate of $\epsilon_{4}$. Indeed, if $\mu_{1,2}(n+j)=0$, then ${ }^{w} x_{n+j}<U_{P}\left(x_{n+j}\right.$ is defined by (2.20)) and since $\left.\psi_{U}\right|_{x_{n+j}} \neq 1$, Lemma 15 implies $\mathrm{I}(\gamma)=0$. In general for any $2 \leq l \leq k-1$, using the unipotent subgroup $x_{(l-1) n+j}$ we deduce from Lemma 15 that $\mu_{1, l}(n+j)=1$. To deduce $\epsilon_{4}(j)=0$ use Lemma 15 with $x_{(k-1) 2 n-n+j}$. This proves $(2.21)$.

Since $w \in H$,

$$
\epsilon_{1}(n-j+1)=\epsilon_{4}(j)=0
$$

Next, we show

$$
\forall 1 \leq l \leq k-1, \quad \mu_{1, l}(n-j+1)=0 .
$$


Indeed, if $\mu_{1, l}(n-j+1)=1$, matrix multiplication implies ${ }^{w} x_{2 n l-n-j+1}<U_{P}$. Since $\left.\psi_{U}\right|_{x_{2 n l-n-j+1}} \neq 1$, it follows from Lemma 15 that $\mathrm{I}(\gamma)=0$.

Now there are two possibilities, $\mu_{1,2}\left(i_{1}\right)=1$ or 0 . Assume the former. Using Lemma 15 as above (the proof of $(2.21)$ ), we conclude that $\mu_{1, l}\left(i_{1}\right)=\epsilon_{1}\left(i_{1}\right)=1$ for all $3 \leq l \leq k-1$. Since $w \in H$, $\epsilon_{1}\left(i_{1}\right)=1$ implies $\epsilon_{4}\left(n-i_{1}+1\right)=1$, and an argument similar to the one used for (2.22) implies that $\mu_{1, l}\left(2 n-i_{1}+1\right)=0$ for all $1 \leq l \leq k-1$. Then we see that ${ }^{w}\left(1, y_{i_{1}, i_{2}}\left(t_{2}\right), \ldots, y_{i_{1}, i_{2}}\left(t_{k-1}\right)\right) \in P$ $\left(i_{2}=n+j\right)$. Therefore, we may assume that $u$ is such that

$$
u^{1}=\left(y_{i_{1}, i_{2}}\left(t_{1}\right) u_{1}^{\prime}, u_{2}^{\prime}, \ldots, u_{k-1}^{\prime}\right) .
$$

Let $x\left(t_{1}\right)=y_{i_{1}, n+j}\left(-t_{1}\right) y_{n-j+1,2 n-i_{1}+1}\left(\zeta t_{1}\right) \in G$, where $\zeta= \pm 1$. For brevity, put $\mathrm{e}\left(x\left(t_{1}\right)\right)=$ $\left(x\left(t_{1}\right), I_{2 n}\right) \in H$. We have

$$
w u=w u^{1} u^{2} \sim w u^{1} u^{2} \mathrm{e}\left(x\left(t_{1}\right)\right)=w u^{1} \mathrm{e}\left(x\left(t_{1}\right)\right) v^{2},
$$

where $v^{2}$ has the same form as $u^{2}\left(\mathrm{e}\left(x\left(t_{1}\right)\right)\right.$ does not commute with $\left.u^{2}\right)$. Also

$$
\begin{aligned}
u^{1} \mathrm{e}\left(x\left(t_{1}\right)\right) & =\left(y_{i_{1}, i_{2}}\left(t_{1}\right) \mathrm{e}\left(x\left(t_{1}\right)\right) u_{1}^{\prime}, \mathrm{e}\left(x\left(t_{1}\right)\right) u_{2}^{\prime}, \ldots, \mathrm{e}\left(x\left(t_{1}\right)\right) u_{k-1}^{\prime}\right) \\
& =\left(x_{n-j+1,2 n-i_{1}+1}\left(t_{1}\right), \mathrm{e}\left(x\left(t_{1}\right)\right), \ldots, \mathrm{e}\left(x\left(t_{1}\right)\right)\right) v^{1} .
\end{aligned}
$$

However, from the structure of $w$ it follows that

$$
{ }^{w}\left(x_{n-j+1,2 n-i_{1}+1}\left(t_{1}\right), \mathrm{e}\left(x\left(t_{1}\right)\right), \ldots, \mathrm{e}\left(x\left(t_{1}\right)\right)\right) \in P .
$$

Hence $w u \sim w v^{1} v^{2}$. This completes the first case $\mu_{1,2}\left(i_{1}\right)=1$.

Now assume $\mu_{1,2}\left(i_{1}\right)=0$. Recall that we assumed

$$
u^{1}=\left(y_{i_{1}, i_{2}}\left(t_{1}\right) u_{1}^{\prime}, y_{i_{1}, i_{2}}\left(t_{2}\right) u_{2}^{\prime}, \ldots, y_{i_{1}, i_{2}}\left(t_{k-1}\right) u_{k-1}^{\prime}\right)
$$

with $t_{1} \neq 0$. Let

$$
x\left(a, t_{1}, t_{2}\right)=x_{n+j, 2 n+i_{1}}(a) x_{i_{1}}\left(-a t_{1}\right) x_{n+j}\left(a t_{2}\right),
$$

where $x_{n+j, 2 n+i_{1}}$ was defined before (2.20). By the definition of $x_{i_{1}}, x_{n+j}, x_{n+j, 2 n+i_{1}}$ and $\psi_{U}$,

$$
\psi_{U}\left(x_{n+j, 2 n+i_{1}}\left(a_{1}\right) x_{i_{1}}\left(a_{2}\right) x_{n+j}\left(a_{3}\right)\right)=\psi_{U}\left(a_{2}+a_{3}\right), \quad \forall a_{i} \in \mathbb{A} \text {. }
$$

It follows from matrix multiplication that one can choose $u^{\prime} \in U$ in such a way that $\psi_{U}\left(u^{\prime}\right)=1$ and

$$
u^{1} x\left(a, t_{1}, t_{2}\right) u^{\prime}=x_{n+j, 2 n+i_{1}}(a) u^{1} .
$$

In fact, $u^{\prime}$ is a product of matrices of the form $x_{2 n+c, d}(\cdot)$ where $1 \leq c<d \leq 2 n$. Thus if $t_{1} \neq t_{2}$,

$$
\psi_{U}\left(x\left(a, t_{1}, t_{2}\right) u^{\prime}\right)=\psi_{U}\left(x\left(a, t_{1}, t_{2}\right)\right)=\psi\left(a\left(t_{2}-t_{1}\right)\right)
$$

which is nontrivial, and also ${ }^{w} x_{n+j, 2 n+i_{1}}<U_{P}$, hence $\mathrm{I}(\gamma)=0$. Therefore, we may assume $t_{1}=t_{2}$. Next consider the value of $\mu_{1,3}\left(i_{1}\right)$. If $\mu_{1,3}\left(i_{1}\right)=1$, we proceed as in the case $\mu_{1,2}\left(i_{1}\right)=1$. If $\mu_{1,3}\left(i_{1}\right)=0$, continue as above to deduce $t_{3}=t_{1}$. Proceeding in this manner we need to consider the case where $u^{1}$ is such that $t_{l}=t_{1}$ for all $2 \leq l \leq k-1$. Finally, if $\epsilon_{1}\left(i_{1}\right)=1$ we proceed as in the case $\mu_{1,2}\left(i_{1}\right)=1$. If $\epsilon_{1}\left(i_{1}\right)=0$, since we established $t_{k-1}=t_{1}$ we can use Lemma 15 with the unipotent subgroup $x_{(k-1) 2 n-n+j}$. This completes the proof of the case $\mu_{1,2}\left(i_{1}\right)=0$ and thereby the case $i_{1} \leq n<i_{2}$.

Proof of Claim 17. Our first step is to prove that if $I(\gamma) \neq 0$, then

$$
\forall 1 \leq l \leq k-1, \quad \mu_{1, l}=\operatorname{diag}\left(\alpha_{l}, 0_{n-j}, \beta_{l}, 0_{j}\right) .
$$

Here $\alpha_{l} \in \mathrm{Mat}_{j}$ and $\beta_{l} \in \mathrm{Mat}_{n-j}$ are diagonal matrices, whose diagonal elements are zeros and ones. Consider the matrix $\mu_{1, l}$, and assume that for some $1 \leq i \leq j$ we have $\mu_{1, l}(2 n-j+i)=1$. 
Then, it follows from matrix multiplication that ${ }^{w} x_{2 n l-n+i}<U_{P}$. Hence by Lemma $15, \mathrm{I}(\gamma)=0$ for this representative. Thus we may assume $\mu_{1, l}=\operatorname{diag}\left(d, 0_{j}\right)$ where $d$ is a diagonal matrix of size $2 n-j$. Similarly, if $\mu_{1, l}(j+i)=1$ for some $1 \leq i \leq n-j$, we use the unipotent subgroup $x_{2 n l+j+i}$ and Lemma 15 to deduce that this representative contributes zero. Thus, we have shown (2.23).

Consider the matrix $\mu_{1,1}$. Assume $\alpha_{1}$ contains $j_{1}$ nonzero entries and $\beta_{1}$ contains $b_{1}$ nonzero entries (these nonzero entries must then be 1 ), where $0 \leq j_{1} \leq j$ and $0 \leq b_{1} \leq n-j$. Using the Weyl elements $\mathrm{e}\left(v_{1}\right), \ldots, \mathrm{e}\left(v_{j-1}\right)$ and $\mathrm{e}\left(v_{j+1}\right), \ldots, \mathrm{e}\left(v_{n-1}\right)$ (see $\left.(2.15)\right)$, we have $w u^{2} \sim w^{\prime} v^{2}$ where $v^{2}$ is a matrix of the form (2.16) with possibly a different matrix $T$, and $w^{\prime}$ is as follows. First, we have

$$
\mu_{1,1}=\operatorname{diag}\left(I_{j_{1}}, 0_{n-j_{1}}, I_{b_{1}}, 0_{n-b_{1}}\right)
$$

(we do not need to use e $\left(v_{j}\right)$ for this), and for $2 \leq l \leq k-1$, the matrices $\mu_{1, l}$ are still of the form (2.23), perhaps with different $\alpha_{l}$ and $\beta_{l}$. Finally, the matrix $\epsilon_{1}$ of both $w$ and $w^{\prime}$ is the same. Re-denote $w=w^{\prime}$ and $u^{2}=v^{2}$.

Next we claim that if $\mathrm{I}\left(w u^{2}\right) \neq 0$, then the first $j_{1}$ (resp., $b_{1}$ ) diagonal entries of $\alpha_{2}$ (resp., $\beta_{2}$ ) are 1, i.e., $\alpha_{2}=\operatorname{diag}\left(I_{j_{1}}, \alpha_{2}^{\prime}\right), \beta_{2}=\operatorname{diag}\left(I_{b_{1}}, \beta_{2}^{\prime}\right)$ and then

$$
\mu_{1,2}=\operatorname{diag}\left(I_{j_{1}}, \alpha_{2}^{\prime}, 0_{n-j}, I_{b_{1}}, \beta_{2}^{\prime}, 0_{j}\right) .
$$

Indeed, suppose $\mu_{1,2}(i)=0$ for some $1 \leq i \leq j_{1}$. Then ${ }^{w} x_{i}<U_{P}$ and we get zero contribution. Similarly for $\beta_{2}$.

Now using multiplication on the right by the Weyl elements e $\left(v_{j_{1}+1}\right), \ldots, \mathrm{e}\left(v_{j-j_{1}-1}\right)$ (if $j_{1}<$ $j-1)$, or $\mathrm{e}\left(v_{j+1}\right), \ldots, \mathrm{e}\left(v_{n-b_{1}-1}\right)$ (if $b_{1}<n-j-1$ ), we deduce $w u^{2} \sim w^{\prime} v^{2}$. Here $\mu_{1,1}$ and $\epsilon_{1}$ are the same for $w$ and $w^{\prime}$, but the matrix $\mu_{1,2}$ of $w^{\prime}$ is

$$
\operatorname{diag}\left(I_{j_{2}}, 0_{n-j_{2}}, I_{b_{2}}, 0_{n-b_{2}}\right),
$$

where $0 \leq j_{1} \leq j_{2} \leq j$ and $0 \leq b_{1} \leq b_{2} \leq n-j$. Again, put $w=w^{\prime}$ and $u^{2}=v^{2}$.

Proceeding this way, we may assume that if $w u^{2}$ is a representative with nonzero contribution, then for some $0 \leq j \leq n$ we have $\epsilon_{1}=\operatorname{diag}\left(I_{j}, 0_{n-j}\right)$, and there are $0 \leq j_{1} \leq \ldots \leq j_{k-1} \leq j$ and $0 \leq b_{1} \leq \ldots \leq b_{k-1} \leq n-j$ such that

$$
\forall 1 \leq l \leq k-1, \quad \mu_{1, l}=\operatorname{diag}\left(I_{j_{l}}, 0_{n-j_{l}}, I_{b_{l}}, 0_{n-b_{l}}\right) .
$$

Also $u^{2}$ has the form (2.16).

Note that in all cases we can assume that $w u^{2} \psi w$, in particular that $u^{2}$ is not the identity matrix, i.e., $T \neq 0$. Otherwise $w u^{2}\left(I_{2 n}, G\right) \cap U_{P}={ }^{w}\left(I_{2 n}, G\right) \cap U_{P}$, and ${ }^{w}\left(I_{2 n}, G\right) \cap U_{P}$ contains the unipotent radical of the Siegel parabolic subgroup of $G$. Hence, since $\pi_{2}$ is cuspidal, $\mathrm{I}(\gamma)=0$ (then the claim is proved).

The last paragraph implies that we can also assume $j<n$, since otherwise $w u^{2} \sim w$.

Next assume $b_{l} \neq 0$, for some $l$. Since $b_{l} \leq b_{k-1}$, we also have $b_{k-1} \neq 0$. The rank of $T$ is at most $n-j$. Further assume that one of the first $b_{k-1}$ columns of $T^{\prime}$ is nonzero. We prove that in this case, $w u^{2}$ contributes zero to the integral. Conjugating by a suitable element in $\mathrm{GL}_{2 k n}(F)$, we may assume that the $(1, l)$-th entry of $T^{\prime}$ is 1 for some $1 \leq l \leq b_{k-1}$. Consider the unipotent element $x(a)=x_{(k-1) 2 n-n+l, 2 k n+1}(a) x_{(k-1) 2 n-n+l}(-a) \in U$. Then $\psi_{U}(x(a)) \neq 1$ and $u^{2} x(a)\left(u^{2}\right)^{-1}=$ $x_{(k-1) 2 n-n+l, 2 k n+1}(a)$. Also since $b_{k-1} \neq 0$ and $1 \leq l \leq b_{k-1}$, we have ${ }^{w} x_{(k-1) 2 n-n+l, 2 k n+1}<U_{P}$. Hence by Lemma 15 we get zero contribution from this element. 
Assuming the first $b_{k-1}$ columns of $T^{\prime}$ are zero, let

$$
v_{0}=\left(\left(\begin{array}{cccc}
I_{n-b_{k-1}} & & & \\
& & I_{b_{k-1}} & \\
& -I_{b_{k-1}} & & I_{n-b_{k-1}}
\end{array}\right), I_{2 n}\right) \in H .
$$

Then $v_{0}$ and $u^{2}$ commute. Also, since $b_{l} \leq b_{k-1}$ for all $l$, then $w v_{0} \sim w^{\prime}$ (because $v_{0} \in G \times G$ ), where in $w^{\prime}$ we have

$$
\mu_{1, l}=\operatorname{diag}\left(I_{j_{l}}, 0_{n-j_{l}-b_{l}}, I_{b_{l}}, 0_{n}\right) .
$$

Here we used the fact that $b_{l} \leq n-j \leq n-j_{l}$ for all $l$, in order to permute each $\mu_{1, l}$ from (2.24) to $(2.25)$ (the last $0_{b_{l}}$ block of $0_{n-j_{l}}$ was permuted to form $0_{n}$ with the last $0_{n-b_{l}}$ block). Multiplying on the left by a suitable permutation matrix in $\mathrm{GL}_{2 k n}$, we deduce $w^{\prime} u^{2} \sim w^{\prime \prime} v^{2}$, where $v^{2}$ is defined as in (2.16) with $j$ replaced by $j^{\prime}$ such that $j<j^{\prime}$, and $T$ is replaced by a matrix of size $\left(n-j^{\prime}\right) \times n$. The matrix $w^{\prime \prime}$ has the structure that

$$
\mu_{1, l}=\operatorname{diag}\left(I_{j_{l}^{\prime}}, 0_{n-j_{l}^{\prime}}, 0_{n}\right)
$$

for some $j_{l}^{\prime} \leq j^{\prime}$.

Therefore, we reduced the structure of $\gamma$ to the form $w u^{2}$ where for some $0 \leq j<n, \mu_{1, l}=$ $\operatorname{diag}\left(I_{j_{l}}, 0_{2 n-j_{l}}\right)$ with $0 \leq j_{l} \leq j$ for all $1 \leq l \leq k-1$, and $u^{2}$ takes the form $(2.16)$.

Proof of Claim 18. We have $0<j<n, \mu_{1, l}=\operatorname{diag}\left(I_{j_{l}}, 0_{2 n-j_{l}}\right)$ and $0 \leq j_{l} \leq j$ for all $1 \leq l \leq k-1$. Also $u^{2}$ is of the form (2.16). First assume $j_{l}>0$ for some $l$, and let $l_{0}$ be the minimal $l$ with this property. Let $V$ be the unipotent radical of the standard parabolic subgroup of $\mathrm{GL}_{2 k n}$ corresponding to the following composition

$$
\left((2 n)^{l_{0}-1}, j_{l_{0}}, j_{l_{0}+1}, \ldots, j_{k-1}, j, b\right)
$$

of $2 \mathrm{kn}$ (here $b$ is uniquely determined by the previous integers). Identify $V /[V, V]$ with the abelian group

$$
\operatorname{Mat}_{2 n} \oplus \ldots \oplus \operatorname{Mat}_{2 n} \oplus \operatorname{Mat}_{j_{0} \times\left(j_{l^{+1}}\right)} \oplus \operatorname{Mat}_{\left(j_{l_{0}+1}\right) \times\left(j_{l_{0}+2}\right)} \oplus \ldots \oplus \operatorname{Mat}_{j_{k-1} \times j} \oplus \operatorname{Mat}_{j \times b} .
$$

Let $X$ be the subgroup of $V /[V, V]$ consisting of vectors such that in their projection into the rightmost component $\mathrm{Mat}_{j \times b}$, the first $b-\left(2 n-j_{l_{0}}\right)$ columns are zero. Define the unipotent group $Y$ as the preimage of $X$ under the quotient map $V \mapsto V /[V, V]$. Define a character of $V /[V, V]$ by multiplying $\psi \circ$ tr of each component. Here tr of a non-square matrix is still defined as the sum of entries on the (principal) diagonal. Pulling back this character to a character of $V$ and restricting to $Y$ yields a character denoted $\psi_{Y}$. We see that $Y$ is contained in $\gamma^{-1} U \cap \mathrm{GL}_{2 k n}$. Thus we obtain the Fourier coefficient

$$
\int_{Y(F) \backslash Y(\mathbb{A})} f(y h, s) \psi_{Y}(y) d y, \quad h \in H(\mathbb{A}),
$$

as an inner integration. We claim that this coefficient vanishes for all data. Indeed, after a suitable conjugation of a subgroup of $Y$ by a Weyl element of $\mathrm{GL}_{2 k n}(F)$ ( $f$ is left-invariant by $\left.\mathrm{GL}_{2 k n}(F)\right)$, we obtain in (2.26) an inner integration

$$
\int_{Y^{\prime}(F) \backslash Y^{\prime}(\mathbb{A})} f\left(y^{\prime} h, s\right) \psi\left(\sum_{i=1}^{k+1} y_{i, i+1}^{\prime}\right) d y^{\prime} .
$$

Here $Y^{\prime}=V_{\left(1^{k+1}, 2 k n-k-1\right)}$. This Fourier coefficient is attached to the unipotent orbit $((k+$ 1) $\left.1^{2 k n-k-1}\right)$. See e.g., [Gin06]. However, the representation $\mathcal{E}_{\tau}$ is attached to the unipotent orbit 
$\left(k^{2 n}\right)$ which is non-comparable with $\left((k+1) 1^{2 k n-k-1}\right)$. Hence this integral and thereby $(2.26)$ is identically zero.

It remains to consider $\gamma$ such that for $w$ we have $0<j<n$ and $j_{l}=0$ for all $l$. This case is omitted here, as it is very similar to the proof of Claim 19 below.

Proof of Claim 19. Here we consider representatives $w u^{2}\left[\left({ }_{I_{l}}{ }^{0}\right)\right]$ with $w=\left({ }_{-I_{2 k n}} I_{2 k n}\right)$ and $0 \leq$ $l<n$. In fact we can assume $l>0$, since in the proof of Claim 17 above we showed $\mathrm{I}(w)=0$ (i.e., $\mathrm{I}\left(w u^{2}\right)=0$ when $u^{2}$ is the identity, see after $\left.(2.24)\right)$.

Put $\gamma_{l}=\mu_{l} w u^{2}\left[\left({ }_{I_{l}}^{0}\right)\right]$ where

$$
\mu_{l}=\operatorname{diag}\left(\left({ }_{I_{l}} I_{n-l}\right), I_{(4 k-2) n},\left({ }_{I_{n-l}}^{I_{l}}\right)\right) \in P
$$

( $\mu_{l}$ is included to simplify the notation of $L_{\gamma_{l}}$ ). The groups $L_{\gamma_{l}}$ are similar to what we obtained for the element $\delta$ defined in (2.18). In particular, inside $G \times G$ we obtain the unipotent subgroup

$$
\left(I_{2 n},\left(\begin{array}{cccc}
I_{n-l} & & & \\
u_{1} & I_{l} & & \\
u_{2} & & I_{l} & \\
u_{3} & u_{2}^{\prime} & u_{1}^{\prime} & I_{n-l}
\end{array}\right)\right) .
$$

The important observation is that $\gamma_{l} U \cap \mathrm{GL}_{2 k n}=V_{\left((2 n)^{k}\right)}$, but the character we obtain on this group is different from the one obtained for the representative $\delta$. To describe it, put $V=V_{\left((2 n)^{k}\right)}$ and identify $V /[V, V]$ with the direct product of $k-1$ copies of Mat $_{2 n}$. Define the character $\psi_{l}$ of $V$ by pulling back the character

$$
\left(X_{1}, \ldots X_{k-1}\right) \mapsto \psi\left(\operatorname{tr}\left(X_{1}\left({ }^{0}{ }_{I_{n+l}}\right)+X_{2}+\ldots+X_{k-1}\right)\right) .
$$

Thus, as an inner integration we obtain the integral

$$
\int_{\left(U \cap U_{P}\right)(\mathbb{A}) V(F) \backslash V(\mathbb{A})} f\left(v \gamma_{l} u_{0}\left(g_{1}, g_{2}\right), s\right) \psi_{l}^{-1}(v) \psi_{U}\left(u_{0}\right) d v d u_{0} .
$$

Consider the subgroup

$$
V^{\prime}=\left\{\left(\begin{array}{ccc}
I_{n-l} & z & \\
& I_{n+l} & \\
& & I_{(k-1) 2 n}
\end{array}\right)\right\}<\mathrm{GL}_{2 k n}
$$

For a fixed $h \in H(\mathbb{A})$, expand the function

$$
a \mapsto \int_{V(F) \backslash V(\mathbb{A})} f\left(v \operatorname{diag}\left(a, I_{(k-1) 2 n}\right) h, s\right) \psi_{l}^{-1}(v) d v, \quad a \in \mathrm{GL}_{2 n}(\mathbb{A}),
$$

along $V^{\prime}(F) \backslash V^{\prime}(\mathbb{A})$. All nontrivial Fourier coefficients correspond to unipotent orbits which are strictly greater than $\left(k^{2 n}\right)$, hence by the definition of the $(k, 2 n)$ representation (Definition 3 part (1)), they all vanish. We are left with the constant term, so that integral (2.28) becomes

$$
\int_{\left(U \cap U_{P}\right)(\mathbb{A})} \int_{V^{\prime}(F) \backslash V^{\prime}(\mathbb{A})} \int_{V(F) \backslash V(\mathbb{A})} f\left(v v^{\prime} \gamma_{l} u_{0}\left(g_{1}, g_{2}\right), s\right) \psi_{l}^{-1}(v) \psi_{U}\left(u_{0}\right) d v d v^{\prime} d u_{0} .
$$


As a function of $g_{2}$, this integral is left invariant under the unipotent radical (2.27): indeed for $v$ of the form $(2.27), \gamma_{l} v=\operatorname{diag}\left(v^{\prime}, v^{\prime *}\right) u^{\prime}$ where

$$
v^{\prime}=\operatorname{diag}\left(\left(\begin{array}{cccc}
I_{n-l} & u_{1}^{\prime} & -u_{2}^{\prime} & \\
& I_{l} & & \\
& & I_{l} & \\
& & & I_{n-l}
\end{array}\right), I_{(k-1) 2 n}\right) \in V^{\prime}
$$

and $u^{\prime} \in U_{P}$, and now we use the left invariance properties of the function $f$ and change variables in $V^{\prime}$. Using this fact we obtain the constant term of $\varphi_{2}$ along (2.27), which is zero because $\pi_{2}$ is cuspidal. Therefore $\mathrm{I}\left(\gamma_{l}\right)=0$ for all $0 \leq l<n$.

\section{Computation of the local Factors With unRamified Data}

Recall that by Theorem 1 and using the same notation, for $\operatorname{Re}(s) \gg 0$,

$$
Z\left(s, \varphi_{1}, \varphi_{2}, f\right)=\int_{G(\mathbb{A})} \int_{U_{0}(\mathbb{A})}\left\langle\varphi_{1}, \pi(g) \varphi_{2}\right\rangle f_{W\left(\mathcal{E}_{\tau}\right)}\left(\delta u_{0}\left(1,{ }^{\iota} g\right), s\right) \psi_{U}\left(u_{0}\right) d u_{0} d g .
$$

Assume $\varphi_{1}$ and $\varphi_{2}$ are decomposable. Then we can write $\left\langle\varphi_{1}, \pi(g) \varphi_{2}\right\rangle=\prod_{\nu} \omega_{\nu}\left(g_{\nu}\right)$, where $\omega_{\nu}$ is a matrix coefficient of $\pi_{\nu}^{\vee}$ for all $\nu$. Let $S$ be a sufficiently large finite set of places of $F$ (which depends only on $\tau$ ), and write $F_{S}, \tau_{S}$, etc., for the product of local factors over the places of $S$. Then if $f$ is decomposable, by Claim 4 we can write

$$
f_{W\left(\mathcal{E}_{\tau}\right)}(h, s)=f_{W\left(\left(\mathcal{E}_{\tau}\right)_{S}\right)}\left(h_{S}, s\right) \prod_{\nu \notin S} f_{W\left(\left(\mathcal{E}_{\tau}\right)_{\nu}\right)}\left(h_{\nu}, s\right) \quad(h \in H(\mathbb{A})) .
$$

Here $f_{W\left(\left(\mathcal{E}_{\tau}\right)_{S}\right)}$ is the composition of a function in the space of the representation $\operatorname{Ind}_{P\left(F_{S}\right)}^{H\left(F_{S}\right)}\left(\left(\mathcal{E}_{\tau}\right)_{S} \delta_{P}^{s}\right)$ with the functional $(2.4)$, and $f_{W\left(\left(\mathcal{E}_{\tau}\right)_{\nu}\right)}$ belongs to the space of $\operatorname{Ind}_{P\left(F_{\nu}\right)}^{H\left(F_{\nu}\right)}\left(W\left(\left(\mathcal{E}_{\tau}\right)_{\nu}\right) \delta_{P}^{s}\right)$ where $W\left(\left(\mathcal{E}_{\tau}\right)_{\nu}\right)$ is the unique $(k, c)$ model of $\left(\mathcal{E}_{\tau}\right)_{\nu}$. Both $f_{W\left(\left(\mathcal{E}_{\tau}\right)_{S}\right)}$ and $f_{W\left(\left(\mathcal{E}_{\tau}\right)_{\nu}\right)}$ are regarded as complex-valued functions. Then

$$
Z\left(s, \varphi_{1}, \varphi_{2}, f\right)=Z_{S}\left(s, \omega_{S}, f_{W\left(\left(\mathcal{E}_{\tau}\right)_{S}\right)}\right) \prod_{\nu \notin S} Z_{\nu}\left(s, \omega_{\nu}, f_{W\left(\left(\mathcal{E}_{\tau}\right)_{\nu}\right)}\right),
$$

where

$$
\begin{aligned}
& Z_{S}\left(s, \omega_{S}, f_{W\left(\left(\mathcal{E}_{\tau}\right)_{S}\right)}\right)=\int_{G\left(F_{S}\right)} \int_{U_{0}\left(F_{S}\right)} \omega_{S}(g) f_{W\left(\left(\mathcal{E}_{\tau}\right)_{S}\right)}\left(\delta_{S} u_{0}\left(1,{ }^{\iota_{S}} g\right), s\right) \psi_{U, S}\left(u_{0}\right) d u_{0} d g \\
& Z_{\nu}\left(s, \omega_{\nu}, f_{W\left(\left(\mathcal{E}_{\tau}\right)_{\nu}\right)}\right)=\int_{G\left(F_{\nu}\right)} \int_{U_{0}\left(F_{\nu}\right)} \omega_{\nu}(g) f_{W\left(\left(\mathcal{E}_{\tau}\right)_{\nu}\right)}\left(\delta_{\nu} u_{0}\left(1,{ }^{\iota_{\nu}} g\right), s\right) \psi_{U, \nu}\left(u_{0}\right) d u_{0} d g .
\end{aligned}
$$

This is the weaker form of an Eulerian integral we can obtain, called an "almost Euler product" by Takeda [Tak14].

In this section we compute the local factors $Z_{\nu}\left(s, \omega_{\nu}, f_{W\left(\left(\mathcal{E}_{\tau}\right)_{\nu}\right)}\right)$ with unramified data. In order to compute the integral for $G \times \mathrm{GL}_{k}$, we shall reduce it to the $\mathrm{GL}_{n} \times \mathrm{GL}_{k}$ integral. The latter will be further reduced to the case of $n=1$, which is computed directly. Throughout this section notation is local and references to the field are omitted (e.g., $\operatorname{Sp}_{2 n}=\operatorname{Sp}_{2 n}(F)$ ). Local fields are of characteristic 0. Representations are always assumed to act on complex vector spaces, and are smooth. Over archimedean fields representations are also assumed to be admissible Fréchet of moderate growth (e.g., an irreducible representation is automatically assumed to have these properties as well). Note that the local representation $\pi_{\nu}$ is irreducible and unitary, $\tau_{\nu}$ is irreducible generic, and an unramified twist of $\tau_{\nu}$ is unitary (usually the 
cuspidal representation $\tau$ is already taken to be unitary, then this twist is trivial and $\tau_{\nu}$ is already unitary). However, parts of the arguments are more convenient to state in a more general context.

3.1. The integrals for $\mathrm{Sp}_{2 n}$ and $\mathrm{SO}_{2 n}$. We present the local integrals for $G=\mathrm{Sp}_{2 n}$ and $\mathrm{SO}_{2 n}$ over a local field $F$. Let $\pi$ be an irreducible representation of $G$ and $\tau$ be an irreducible, generic representation of $\mathrm{GL}_{k}$.

We now consider two possible cases for the representation $\tau$ : these are the cases relevant for the study of the integrals on the right hand side of (3.1). In the first case $\tau$ is a component at a place $\nu$ of an irreducible cuspidal automorphic representation $\Upsilon$ of $\mathrm{GL}_{k}(\mathbb{A})$, and define $\rho_{c}(\tau)=\left(\mathcal{E}_{\Upsilon}\right)_{\nu}$. The representation $\rho_{c}(\tau)$ affords at least one $(k, c)$ model $W\left(\rho_{c}(\tau)\right)$, which we denote for brevity $W_{c}(\tau)$ (recall $c=2 n$ ).

In the second case $F$ is $p$-adic and $\tau$ is irreducible, generic and unramified. Write $\tau=$ $\operatorname{Ind}_{B_{\mathrm{GL}_{k}}}^{\mathrm{GL}_{k}}\left(\chi_{1} \otimes \ldots \otimes \chi_{k}\right)$, then by Claim 10 the representation $\sigma_{k, c}=\operatorname{Ind}_{P_{\left(c^{k}\right)}}^{\mathrm{GL}_{k c}}\left(\chi_{1} \otimes \ldots \otimes \chi_{k}\right)$ is $(k, c)$. The representation $\sigma_{k, c}$ might be reducible, but it is irreducible when the parameters $\chi_{i}$ are in "general position". Either way, we let $\rho_{c}(\tau)$ be the unique irreducible constituent of $\sigma_{k, c}$ which is $(k, c)$.

When the local integrals arise as the integrals in the decomposition (3.1) at $\nu \notin S$, by Claim 9 both cases above coincide and $\rho_{c}(\tau)=\sigma_{k, c}$, which affords a unique $(k, c)$ model $W_{c}(\tau)$.

Recall that $H=\mathrm{Sp}_{2 k c}$ if $G=\mathrm{Sp}_{2 n}$, and $H=\mathrm{SO}_{2 k c}$ when $G=\mathrm{SO}_{2 n}$. Let $P=M_{P} \ltimes U_{P}$ be the Siegel parabolic subgroup of $H$ with $M_{P}=\left\{\operatorname{diag}\left(g, g^{*}\right): g \in \mathrm{GL}_{k c}\right\}$. Also fix a maximal compact subgroup $K_{H}$ in $H$.

The local integral takes the form

$$
Z\left(s, \omega, f_{W_{c}(\tau)}\right)=\int_{G} \int_{U_{0}} \omega(g) f_{W_{c}(\tau)}\left(\delta u_{0}\left(1,{ }^{\iota} g\right), s\right) \psi_{U}\left(u_{0}\right) d u_{0} d g .
$$

Here $\omega$ is a matrix coefficient of $\pi^{\vee} ; f_{W_{c}(\tau)}$ belongs to the space of the representation $\operatorname{Ind}_{P}^{H}\left(W_{c}(\tau)\right)$, and is regarded as a complex-valued function; $h \mapsto f_{W_{c}(\tau)}(h, s)$ is the unique extension of $f_{W_{c}(\tau)}$ to a standard section of $\operatorname{Ind}_{P}^{H}\left(W_{c}(\tau) \delta_{P}^{s}\right)$ (i.e., its restriction to $K_{H}$ is independent of $s$, see e.g., $\left[\right.$ Wal03, $\S$ IV.1]); $\delta=\delta_{0} \delta_{1}$ with

$$
\delta_{0}=\left(\begin{array}{ll} 
& I_{k c} \\
\epsilon_{0} I_{k c} &
\end{array}\right), \quad \delta_{1}=\left(\begin{array}{cccccc}
I_{(k-1) c} & & & & & \\
& I_{n} & & -\epsilon_{0} I_{n} & & \\
& & I_{n} & & I_{n} & \\
& & & I_{n} & & \\
& & & & I_{n} & \\
& & & & & I_{(k-1) c}
\end{array}\right), \quad \epsilon_{0}= \begin{cases}-1 & G=\mathrm{Sp}_{2 n}, \\
1 & G=\mathrm{SO}_{2 n}\end{cases}
$$

the unipotent subgroup $U_{0}$, the restriction of $\psi_{U}$ to $U_{0},(1, g)$ and $\iota$ are defined by

$$
\begin{aligned}
& U_{0}=\left\{\left(\begin{array}{cccc}
I_{(k-1) c} & & X & Z \\
& I_{c} & & X^{\prime} \\
& & I_{c} & \\
& & & I_{(k-1) c}
\end{array}\right) \in H\right\} \quad\left(\begin{array}{c}
{ }^{t} Z J_{(k-1) c}+\epsilon_{0} J_{(k-1) c} Z=0 \\
X^{\prime}=-\epsilon_{0} J_{c}{ }^{t} X J_{(k-1) c}
\end{array}\right), \\
& \psi_{U}\left(u_{0}\right)=\psi\left(\operatorname{tr}\left(\left(\begin{array}{ll}
0 & I_{n}
\end{array}\right) X\left(\begin{array}{c}
0 \\
I_{n}
\end{array}\right)\right)\right), \\
& (1, g)=\operatorname{diag}\left(I_{(k-1) c+n}, g, I_{n+(k-1) c}\right), \quad \iota=\left({ }_{-\epsilon_{0} I_{n}}^{I_{n}}\right) \text {. }
\end{aligned}
$$


The integral, at least formally, can be regarded as a morphism in the space

$$
\operatorname{Hom}_{G \times G}\left(J_{U, \psi_{U}^{-1}}\left(\operatorname{Ind}_{P}^{H}\left(W_{c}(\tau) \delta_{P}^{s}\right)\right), \pi^{\vee} \otimes \pi\right) .
$$

Here $J_{U, \psi_{U}^{-1}}(\cdots)$ is the twisted Jacquet module with respect to $U$ and $\psi_{U}^{-1}$, regarded as a representation of $G \times G$ by virtue of the embedding $\left(g_{1}, g_{2}\right)$. This follows from the construction and can be verified directly.

Theorem 21. The integrals $Z\left(s, \omega, f_{W_{c}(\tau)}\right)$ satisfy the following properties.

(1) They are absolutely convergent in a right half-plane $\operatorname{Re}(s) \gg 0$ depending only on the representations.

(2) Over non-archimedean fields, one can choose data $\left(\omega, f_{W_{c}(\tau)}\right)$ such that the integral is absolutely convergent and equals 1 , for all s. Over archimedean fields, for any s there is data $\left(\omega, f_{W_{c}(\tau)}\right)$ where $f_{W_{c}(\tau)}$ is $K_{G}$-finite, such that the integral is holomorphic and nonzero in a neighborhood of $s$.

(3) They admit meromorphic continuation to the plane, and over a non-archimedean field with residue cardinality $q$, the continuation is a rational function in $q^{-s}$, i.e., belongs to $\mathbb{C}\left(q^{-s}\right)$.

Proof. We provide only a sketch of the proof, because very similar statements can be found in numerous places in the literature, e.g., [GJ72, JPSS83, GPSR87, BG92, Sou93, Sou95, GRS98, LR05, Kap13b, Kap13c, KM].

Over non-archimedean fields, convergence follows from the following observations: if $\pi$ is supercuspidal, the matrix coefficient is compactly supported modulo the center; in the general case, one can write $\omega$ as a sum of products of matrix coefficients of the representations appearing in the cuspidal support of $\pi^{\vee}$, as in [GJ72]; the Iwasawa decomposition can then be used to reduce to an integral over the torus of $G$; functions in $W_{c}(\tau)$ vanish on torus elements outside a cone, similarly to Whittaker functions (see $[$ Cas $80, \S 6]$ ); the unipotent integration is handled as in $[$ Sou93, § 4].

Part (2) is shown by selecting a section $f$ which is supported in the open orbit $P \delta U(G, G)$, such that the function $\left(u_{0}, g\right) \mapsto f\left(\delta u_{0}\left(1,{ }^{\imath} g\right), s\right)$ on $U_{0} \times G$ vanishes outside the product of compact neighborhoods in $U_{0}$ and $G$. The compact neighborhood $\mathcal{N}_{G}$ in $G$ can be taken to be sufficiently small, such that $\omega$ is constant on $\mathcal{N}_{G}$. See $[R S 05, \S 4]$. The argument on the support also implies absolute convergence.

Regarding meromorphic continuation one can use Bernstein's continuation principle (in [Ban98]), which also implies the rationality statement. To apply this principle we need the following uniqueness result: outside a finite set of values of $q^{-s}$, the space (3.2) is at most one-dimensional. The proof of this result is analogous to the global unfolding of the integral (for $k=1$ this uniqueness was proved in [HKS96]). According to (1), in a right half-plane the integral can be regarded as a morphism in (3.2). Combining the uniqueness result with (2), the meromorphic continuation follows.

Over archimedean fields the proof of (2) is similar. The meromorphic continuation is more difficult, because Bernstein's result is not applicable, but one can argue directly by reducing the integral over $G$ to an integral over a torus, then using the Dixmier-Malliavin Lemma [DM78] and asymptotic results as in [Sou95] (see [GRS98, § 3.2]). See also [KM] for an asymptotic expansion of matrix coefficients.

3.2. The integrals for $\mathrm{GL}_{n}$. As mentioned in $\S 2.1$, the global and hence local integrals can also be defined for general linear groups. As we show in Lemma 27 below, the $G \times \mathrm{GL}_{k}$ integral reduces to a $\mathrm{GL}_{n} \times \mathrm{GL}_{k}$ integral. We therefore define this integral, in a purely local context, where it will be needed. 
Let $\pi$ be an irreducible representation $\mathrm{GL}_{n}$, and $\tau$ and $\tau^{\prime}$ be irreducible generic representations of $\mathrm{GL}_{k}$ such that the central character of $\tau$ is the inverse of the central character of $\tau^{\prime}$ (e.g., $\left.\tau^{\prime}=\tau^{\vee}\right)$. The representation $\rho_{n}(\tau)$ is defined as in $\S 3.1$, either as $\left(\mathcal{E}_{\Upsilon}\right)_{\nu}$ when $\tau$ is assumed to be a component of a cuspidal representation $\Upsilon$, or as the unique irreducible $(k, n)$ constituent of $\sigma_{k, n}=\operatorname{Ind}_{P_{\left(n^{k}\right)}}^{\mathrm{GL}_{k n}}\left(\chi_{1} \otimes \ldots \otimes \chi_{k}\right)$. We similarly define $\rho_{n}\left(\tau^{\prime}\right)$. Note that for the applications in this work, one can simply take $\tau^{\prime}=\tau^{\vee}$.

Put $G=\mathrm{GL}_{n}, H=\mathrm{GL}_{2 k n}$ and $P=P_{(k n, k n)}$. Then $M_{P}=M_{(k n, k n)}$ and $U_{P}=V_{(k n, k n)}$. Let $Q=M \ltimes U$ be the standard parabolic subgroup of $H$ with $M=M_{\left(n^{k-1}, 2 n, n^{k-1}\right)}$. To define $\psi_{U}$, note that $U$ contains a top left and bottom right copies of $V_{\left(n^{k-1}\right)}$. The character $\psi_{U}$ is given by the inverse of (2.3) on each copy of $V_{\left(n^{k-1}\right)}$, and if the middle $4 n \times 4 n$ block of $u \in U$ is written in the form

$$
\left(\begin{array}{cccc}
I_{n} & u_{1} & u_{2} & u_{3} \\
& I_{n} & & u_{4} \\
& & I_{n} & u_{5} \\
& & & I_{n}
\end{array}\right), \quad u_{i} \in \operatorname{Mat}_{n}
$$

then $\psi_{U}$ restricts to the character $\psi\left(\operatorname{tr}\left(-u_{1}+u_{4}\right)\right)$. The embedding of $G \times G$ in $H$ is defined by

$$
\left(g_{1}, g_{2}\right)=\operatorname{diag}\left(g_{1}, \ldots, g_{1}, g_{1}, g_{2}, g_{1}, \ldots, g_{1}\right), \quad g_{1}, g_{2} \in G
$$

where $g_{1}$ appears $k$ times on the left of $g_{2}$, and $k-1$ times on the right. The $\mathrm{GL}_{n} \times \mathrm{GL}_{k}$ integral is

$$
Z\left(s, \omega, f_{W_{n}(\tau) \otimes W_{n}\left(\tau^{\prime}\right)}\right)=\int_{G} \int_{U_{0}} \omega(g) f_{W_{n}(\tau) \otimes W_{n}\left(\tau^{\prime}\right)}\left(\delta u_{0}(1, g), s\right) \psi_{U}\left(u_{0}\right) d u_{0} d g .
$$

Here $\omega$ is a matrix coefficient of $\pi^{\vee}$; the section $h \mapsto f_{W_{n}(\tau) \otimes W_{n}\left(\tau^{\prime}\right)}(h, s)$ is on

$$
\begin{aligned}
& \operatorname{Ind}_{P}^{H}\left(\left(W_{n}(\tau) \otimes W_{n}\left(\tau^{\prime}\right)\right) \delta_{P}^{s}\right) ; \\
& \delta=\delta_{0} \delta_{1}, \quad \delta_{0}=\left(\begin{array}{cc} 
& I_{k n} \\
I_{k n} &
\end{array}\right), \quad \delta_{1}=\left(\begin{array}{llll}
I_{(k-1) n} & & & \\
& I_{n} & I_{n} & \\
& & I_{n} & \\
& & & I_{(k-1) n}
\end{array}\right), \\
& U_{0}=U \cap U_{P}=\left\{\left(\begin{array}{cccc}
I_{(k-1) n} & & X & Z \\
& I_{n} & & Y \\
& & I_{n} & \\
& & & I_{(k-1) n}
\end{array}\right)\right\} \text {, } \\
& \psi_{U}\left(u_{0}\right)=\psi\left(\operatorname{tr}\left(Y\left(\begin{array}{c}
I_{n} \\
0
\end{array}\right)\right)\right) .
\end{aligned}
$$

We may also set $c=n$ in this case, to unify the notation. To avoid confusion, we will usually defer from this and write $n$ explicitly.

The immediate analog of Theorem 21 applies to the $\mathrm{GL}_{n} \times \mathrm{GL}_{k}$ integral. In particular over non-archimedean fields, it is absolutely convergent in a right half-plane, can be regarded as an element of

$$
\operatorname{Hom}_{G \times G}\left(J_{U, \psi_{U}^{-1}}\left(\operatorname{Ind}_{P}^{H}\left(\left(W_{n}(\tau) \otimes W_{n}\left(\tau^{\prime}\right)\right) \delta_{P}^{s}\right)\right), \pi^{\vee} \otimes \pi\right),
$$

and admits meromorphic continuation which belongs to $\mathbb{C}\left(q^{-s}\right)$. 
3.3. Preliminaries for the unramified computation. Henceforth until the end of the paper, let $F$ be a non-archimedean local field with residue cardinality $q, \mathcal{O}$ be its ring of integers, $\varpi \in \mathcal{O}$ be a uniformizer, and normalize the absolute value so that $|\varpi|=q^{-1}$. Let $\psi$ be a nontrivial additive character of $F$, and assume it is unramified, i.e., its conductor is 0 . We choose a Haar measure on $F$ which is self-dual with respect to $\psi$, in particular it assigns the volume 1 to $\mathcal{O}$.

We fix hyperspecial maximal compact subgroups: $K_{\mathrm{GL}_{l}}=\mathrm{GL}_{l}(\mathcal{O}), K_{G}=G(\mathcal{O})$ and $K_{H}=$ $H(\mathcal{O})$, where $G$ is either $\mathrm{Sp}_{2 n}, \mathrm{SO}_{2 n}$ or $\mathrm{GL}_{n}$ and $H$ is defined according to $G$. These choices satisfy the compatibility conditions $\left(K_{G}, K_{G}\right)<K_{H}, K_{\mathrm{GL}_{k c}}=M_{P} \cap K_{H}$ and if $G \neq \mathrm{GL}_{n},{ }^{\iota} K_{G}=$ $K_{G}$. The measures of $K_{G}$ and $K_{H}$ are normalized to be 1 .

For any irreducible unramified representations $\sigma$ and $\tau$ of $\mathrm{GL}_{N}$ and $\mathrm{GL}_{k}$ (resp.), the $L$ function $L(s, \sigma \times \tau)$ was defined in [JS81, JPSS83]. If $t_{\sigma}$ and $t_{\tau}$ are the Satake parameters of $\sigma$ and $\tau$, regarded as representatives of the semi-simple conjugacy classes in $\mathrm{GL}_{N}(\mathbb{C})$ and $\mathrm{GL}_{k}(\mathbb{C})$ associated to $\sigma$ and $\tau$,

$$
L(s, \sigma \times \tau)=\operatorname{det}\left(1-t_{\sigma} \otimes t_{\tau} q^{-s}\right)^{-1} .
$$

Moreover, for any finite dimensional representation $\kappa$ of $\mathrm{GL}_{k}(\mathbb{C})$, define

$$
L(s, \tau, \kappa)=\operatorname{det}\left(1-\kappa\left(t_{\tau}\right) q^{-s}\right)^{-1} \text {. }
$$

In particular by definition

$$
L(s, \tau \times \tau)=L\left(s, \tau, \operatorname{Sym}^{2}\right) L\left(s, \tau, \wedge^{2}\right),
$$

where $\mathrm{Sym}^{2}$ is the symmetric square and $\wedge^{2}$ is the exterior square representation. This equality actually holds for any irreducible admissible representation $\tau$ by Shahidi [Sha92, Corollary 8.2]. Also denote $L(s, \tau)=L(s, \tau$, id $)$, where id is the identity representation of $\mathrm{GL}_{k}(\mathbb{C})$.

Let $G$ be either $\mathrm{Sp}_{2 n}$ or $\mathrm{SO}_{2 n}$. Recall that if $G=\mathrm{Sp}_{2 n}$, then ${ }^{L} G=\mathrm{SO}_{2 n+1}(\mathbb{C})$ and we set $N=2 n+1$, and if $G=\mathrm{SO}_{2 n}$, then ${ }^{L} G=\mathrm{SO}_{2 n}(\mathbb{C})$ and $N=2 n$. Assume that $\pi$ is an irreducible unramified representation of $G$. Let $\Pi$ be the lift of $\pi$ to $\mathrm{GL}_{N}$, obtained using the Satake isomorphism ([Sat63, Bor79]). The representation $\Pi$ is the irreducible unramified representation of $\mathrm{GL}_{N}$ whose Satake parameter is the transfer of the parameter of $\pi$ under the natural embedding ${ }^{L} G \rightarrow \mathrm{GL}_{N}(\mathbb{C})$. Then by definition $L(s, \pi \times \tau)=L(s, \Pi \times \tau)$.

Furthermore, let $R=M_{R} \ltimes U_{R}$ be a Siegel parabolic subgroup of $G$. One can choose an irreducible unramified principal series representation $\pi_{n}^{\prime}$ of $\mathrm{GL}_{n} \cong M_{R}$ such that $\pi$ is the irreducible unramified constituent of $\operatorname{Ind}_{R}^{G}\left(\pi_{n}^{\prime \vee}\right)$. Then the definition implies

$$
L(s, \pi \times \tau)=[L(s, \tau)] L\left(s, \pi_{n} \times \tau\right) L\left(s, \pi_{n}^{\vee} \times \tau\right),
$$

where $L(s, \tau)$ appears only when $G=\operatorname{Sp}_{2 n}$.

3.4. Local decomposition of $(k, c)$ functionals. Let $k$ and $c$ be positive integers. We describe a realization of local $(k, c)$ functionals. Let

$$
\tau=\operatorname{Ind}_{B_{\mathrm{GL}_{k}}}^{\mathrm{GL}_{k}}\left(\chi_{1} \otimes \ldots \otimes \chi_{k}\right), \quad \sigma_{k, c}=\operatorname{Ind}_{P_{\left(c^{k}\right)}}^{\mathrm{GL}_{k c}}\left(\chi_{1} \otimes \ldots \otimes \chi_{k}\right)
$$

where $\tau$ is assumed to be irreducible and $\chi_{1}, \ldots, \chi_{k}$ are unramified quasi-characters of $F^{*}$. We do not assume at this point that $\sigma_{k, c}$ is irreducible. According to Claim 10, the space of $(k, c)$ functionals on $\sigma_{k, c}$ is one-dimensional. We construct such a functional using the Jacquet integral.

Put

$$
w_{k, c}=\left({ }_{I_{c}}{ }^{I_{c}{ }^{I_{c}}}\right) \in \mathrm{GL}_{k c} .
$$


The following defines a $(k, c)$ functional on $\sigma_{k, c}$ :

$$
\xi \mapsto \int_{V_{\left(c^{k}\right)}} \xi\left(w_{k, c} v\right) \psi^{-1}(v) d v .
$$

Here $\xi$ belongs to the space of $\sigma_{k, c}$, and $\psi$ is defined by (2.3). Twisting the inducing data using auxiliary complex parameters, i.e., replacing $\chi_{i}$ by ||$_{i} \chi_{i}$ with $\zeta_{i} \in \mathbb{C}$ for $1 \leq i \leq k$, there is a cone where the integral is absolutely convergent (the proof is identical to the proof for the similar intertwining integral, see e.g., [Sha81, § 2]). We can also choose data such that (3.4) is absolutely convergent and equals 1 , for all choices of $\zeta_{i}$, namely a function with support in $P_{\left(c^{k}\right)} w_{k, c} \mathcal{N}$, where $\mathcal{N}$ is a small compact open neighborhood of the identity in $\mathrm{GL}_{k c}$. Since the space of $(k, c)$ functionals on $\sigma_{k, c}$ is one-dimensional, Bernstein's continuation principle (in [Ban98]) implies that (3.4) admits analytic continuation in the parameters $\zeta_{i}$, and it also follows (by the aforementioned choice of data) that it is a nonzero functional for all $\zeta_{i}$, in particular on $\sigma_{k, c}$ when setting $\zeta_{1}=\ldots=\zeta_{k}=0$.

Let $0 \subset \mathbb{V}_{1} \subset \ldots \subset \mathbb{V}_{l} \subset \sigma_{k, c}$ be a Jordan-Hölder series of $\sigma_{k, c}$, and $i$ be minimal such that (3.4) does not vanish on $\mathbb{V}_{i}\left(i\right.$ exists because the functional does not vanish on $\left.\sigma_{k, c}\right)$. Then (3.4) restricts to a nonzero functional on $\mathbb{V}_{i}$ and factors through the quotient $\mathbb{V}_{i-1} \mid \mathbb{V}_{i}$. Since the Jacquet functor is exact and the dimension of $J_{V_{\left(c^{k}\right)}, \psi}\left(\sigma_{k, c}\right)$ is $1, \mathbb{V}_{i-1} \mid \mathbb{V}_{i}$ is the unique irreducible constituent of $\sigma_{k, c}$ affording a $(k, c)$ functional, and we denote it by $\rho_{c}(\tau)$. The corresponding $(k, c)$ model of $\rho_{c}(\tau)$ is denoted $W_{c}(\tau)$; it is isomorphic to $\rho_{c}(\tau)$ and a summand of the $(k, c)$ model of $\sigma_{k, c}$.

We describe a decomposition result for the functional (3.4) on $\sigma_{k, c}$. For simplicity, the dependence on the twisting parameters $\zeta_{i}$ is omitted from the notation. Assume $c=a+b$ with $a, b \geq 1$ and put $V=V_{\left(c^{k}\right)}$. As in $\S 2.2$, denote $v \in V_{\left(c^{k}\right)}$ by $v=\left(v_{i, j}\right)_{1 \leq i, j \leq k}$, where $v_{i, j} \in \mathrm{Mat}_{c}$. We rewrite the blocks $v_{i, j}$ of $v \in V$ in the form

$$
v_{i, j}=\left(\begin{array}{cc}
v_{i, j}^{1} & v_{i, j}^{2} \\
v_{i, j}^{3} & v_{i, j}^{4}
\end{array}\right), \quad v_{i, j}^{1} \in \operatorname{Mat}_{a}, \quad v_{i, j}^{4} \in \text { Mat }_{b} .
$$

For $t=1, \ldots, 4$, let $V^{t}$ be the subgroup consisting of the matrices $v \in V$ such that in each block $v_{i, j}$ with $i<j$, the coordinates of $v_{i, j}^{t^{\prime}}$ are zero for all $t^{\prime} \neq t$. Also define for any $a, b \geq 1$,

$$
l_{a, b}=\left(\begin{array}{cccccccc}
I_{a} & & & & & & & \\
0 & 0 & I_{a} & & & & & \\
0 & 0 & 0 & 0 & I_{a} & \ddots & & \\
& & & & & & I_{a} & 0 \\
0 & I_{b} & & & & & & \\
0 & 0 & 0 & I_{b} & & \ddots & & \\
& & & & & 0 & I_{b}
\end{array}\right) \in \mathrm{GL}_{k(a+b)} .
$$

For example if $k=3, a=2$ and $b=3$,

$$
l_{2,3}=\left(\begin{array}{cccc}
I_{2} & & & \\
& I_{2} & & \\
& I_{3} & & I_{2} \\
& & I_{3} & \\
& & & I_{3}
\end{array}\right) .
$$

Lemma 22. For $a, b \geq 1$ such that $c=a+b$, and for any $\xi$ in the space of $\sigma_{k, c}$,

$$
\int_{V_{\left(c^{k}\right)}} \xi\left(w_{k, c} v\right) \psi^{-1}(v) d v=\int_{V^{3}} \xi_{W_{a}(\tau) \otimes W_{b}(\tau)}\left(l_{a, b} v\right) d v,
$$


where $\xi_{W_{a}(\tau) \otimes W_{b}(\tau)}$ is defined by (3.11) below and belongs to the space of the representation

$$
\operatorname{Ind}_{P_{(k a, k b)}}^{\mathrm{GL}_{k c}}\left(\left(W_{a}(\tau) \otimes W_{b}(\tau)\right) \delta_{P_{(k a, k b)}}^{-1 /(2 k)}\right) .
$$

This equality is valid in the domain where (3.4) is absolutely convergent and in general by meromorphic continuation.

Proof. First note that

$$
w_{k, c}={ }_{a, b}^{-1} \operatorname{diag}\left(w_{k, a}, w_{k, b}\right)
$$

(see (2.11) for our notation regarding conjugations). Write the integral over $V_{\left(c^{k}\right)}$ as an iterated integral $d V^{2} d V^{1} d V^{4} d V^{3}$. We have

$$
w_{k, c} V_{\left(c^{k}\right)}=l_{a, b}^{-1} \operatorname{diag}\left(w_{k, a}, w_{k, b}\right) l_{a, b} V^{2} \operatorname{diag}\left(w_{k, a}, w_{k, b}\right)^{l_{a, b}}\left(V^{1} V^{4}\right) l_{a, b} V^{3} .
$$

The character $\psi$ is trivial on $V^{2}$ and $V^{3}$. Then (3.4) becomes

$\int_{V^{3}} \int_{V^{4}} \int_{V^{1}} \int_{V^{2}} \xi\left(l_{a, b}^{-1}\left({ }^{\operatorname{diag}\left(w_{k, a}, w_{k, b}\right) l_{a, b}} v^{2}\right) \operatorname{diag}\left(w_{k, a}{ }^{l_{a, b}} v^{1}, w_{k, b}{ }^{l_{a, b}} v^{4}\right) l_{a, b} v^{3}\right) \psi^{-1}\left(v^{1}\right) \psi^{-1}\left(v^{4}\right) d v^{2} d v^{1} d v^{4} d v^{3}$.

Denote, for any $\xi$ in the space of $\sigma_{k, c}$,

$$
T_{l_{a, b}} \xi(g)=\int_{V^{2}} \xi\left(l_{a, b}^{-1}\left(\operatorname{diag}\left(w_{k, a}, w_{k, b}\right) l_{a, b} v^{2}\right) g\right) d v^{2} \quad\left(g \in \mathrm{GL}_{k c}\right) .
$$

For $A_{i} \in \mathrm{GL}_{a}$ and $B_{i} \in \mathrm{GL}_{b}$,

$$
\begin{aligned}
& T_{l_{a, b}} \xi\left(\operatorname{diag}\left(A_{1}, \ldots, A_{k}, B_{1}, \ldots, B_{k}\right)\right) \\
& =\delta_{P_{\left(c^{k}\right)} / 2}^{1 / 2}\left(\operatorname{diag}\left(A_{1}, B_{1}, \ldots, A_{k}, B_{k}\right)\right) \\
& \quad \times \prod_{i=1}^{k} \chi_{i}\left(\operatorname{det} A_{i}\right) \chi_{i}\left(\operatorname{det} B_{i}\right) \prod_{i=1}^{k}\left|\operatorname{det} A_{k-i+1}\right|^{b(k-i)}\left|\operatorname{det} B_{i}\right|^{-a(k-i)} T_{l_{a, b}} \xi\left(I_{k c}\right) .
\end{aligned}
$$

Then if $A=\operatorname{diag}\left(A_{1}, \ldots, A_{k}\right), B=\operatorname{diag}\left(B_{1}, \ldots, B_{k}\right)$ and $\chi(A)=\prod_{i=1}^{k} \chi_{i}\left(\operatorname{det} A_{i}\right)$,

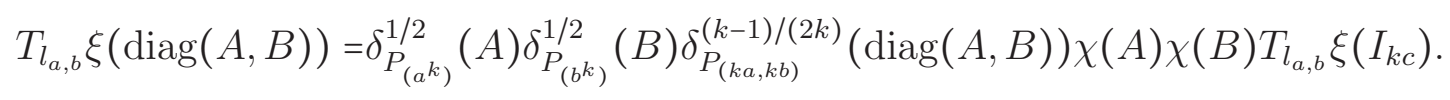

Also $T_{l_{a, b}} \xi(u g)=T_{l_{a, b}} \xi(g)$ for $u \in V_{(k a, k b)}$. Therefore $T_{l_{a, b}}$ is an intertwining operator from the space of $\sigma_{k, c}$ to the space of the representation

$$
\operatorname{Ind}_{P_{(k a, k b)}}^{\mathrm{GL}_{k c}}\left(\left(\sigma_{a, k} \otimes \sigma_{b, k}\right) \delta_{P_{(k a, k b)}}^{-1 /(2 k)}\right) .
$$

Now (3.7) becomes

$$
\int_{V^{3}} \int_{V^{4}} \int_{V^{1}} T_{l_{a, b}} \xi\left(\operatorname{diag}\left(w_{k, a}^{l_{a, b}} v^{1}, w_{k, b}^{l_{a, b}} v^{4}\right) l_{a, b} v^{3}\right) \psi^{-1}\left(v^{1}\right) \psi^{-1}\left(v^{4}\right) d v^{1} d v^{4} d v^{3} .
$$

The integrals $d v^{1} d v^{4}$ constitute the applications of $(k, a)$ and $(k, b)$ functionals, e.g., ${ }^{l_{a, b}} V^{1}=$ $\operatorname{diag}\left(V_{\left(a^{k}\right)}, I_{k b}\right)$. Hence if

$$
\xi_{W_{a}(\tau) \otimes W_{b}(\tau)}(g)=\int_{V^{4}} \int_{V^{1}} T_{l_{a, b}} \xi\left(\operatorname{diag}\left(w_{k, a^{l a b b}} v^{1}, w_{k, b}{ }^{l_{a, b}} v^{4}\right) g\right) \psi^{-1}\left(v^{1}\right) \psi^{-1}\left(v^{4}\right) d v^{1} d v^{4},
$$


the function $\xi_{W_{a}(\tau) \otimes W_{b}(\tau)}$ belongs to the space of (3.6). Integral (3.10) is equal to

$$
\int_{V^{3}} \xi_{W_{a}(\tau) \otimes W_{b}(\tau)}\left(l_{a, b} v^{3}\right) d v^{3},
$$

as claimed.

Corollary 23. Assume $1-q^{-s} \chi_{i}(\varpi) \chi_{j}^{-1}(\varpi) \neq 0$ for $\operatorname{Re}(s) \geq 1$, for all $i \neq j$. Then the functional (3.4) is nonzero on the normalized unramified vector $\xi$ in the space of $\sigma_{k, c}$. Note that the assumption always holds if we consider ||$\zeta_{i} \chi_{i}$ instead of $\chi_{i}$ and take $\zeta_{i} \gg \zeta_{j}$ for all $i<j$.

Proof. We use induction on $c$. For $c=1$, the functional (3.4) is the usual Whittaker functional given by a Jacquet integral. Since $\sigma_{k, 1}=\tau$ which is irreducible, this functional is nonzero on $\xi$ by the Casselman-Shalika formula [CS80] and the irreducibility criterion for principal series representations (e.g., [BZ77, Cas80]).

Assume $c>1$ and apply Lemma 22 using $a=1$ and $b=c-1$. Conjugating $V^{3}$ by $l_{1, c-1}$, we obtain

$$
\int_{V^{3}} \xi_{W_{1}(\tau) \otimes W_{c-1}(\tau)}\left({ }^{l_{1, c-1}} v\right) d v
$$

We will show that the coordinates of $v$ can be assumed to lie in $\mathcal{O}$. If the coordinates of $v$ are given by (3.5), i.e., the nontrivial coordinates of $v$ are the blocks $v_{i, j}^{3}$ where $v_{i, j}^{3} \in \operatorname{Mat}_{c-1 \times 1}$, then

$$
l_{1, c-1} v=\left(\begin{array}{cc}
I_{k} & \\
{[v]} & I_{k(c-1)}
\end{array}\right), \quad[v]=\left(\begin{array}{cccc}
0 & v_{1,2}^{3} & \cdots & v_{1, k}^{3} \\
\vdots & \ddots & \ddots & \vdots \\
\vdots & & & v_{k-1, k}^{3} \\
0 & \cdots & \cdots & 0
\end{array}\right)
$$

(Direct matrix multiplication.) Consider matrices of the form

$$
\left(\begin{array}{cc}
I_{k} & {[x]} \\
& I_{k(c-1)}
\end{array}\right), \quad[x]=\left(\begin{array}{cccc}
0 & 0 & \cdots & 0 \\
\vdots & x_{1,2} & \ddots & \vdots \\
\vdots & \vdots & \ddots & 0 \\
0 & x_{1, k} & \cdots & x_{k-1, k}
\end{array}\right), \quad x_{i, j} \in \operatorname{Mat}_{1 \times c-1}
$$

For each $1 \leq i \leq k-1$ and $2 \leq j \leq k$, let $\mathcal{X}_{i, j}$ be the subgroup consisting of matrices of this form where the coordinates of $[x]$ are zero except at the block $x_{i, j}$, which takes arbitrary coordinates in $\mathcal{O}$. Starting with $i=k-1$ and $j=k$, for $x \in \mathcal{X}_{i, j}$ we have

$$
\xi_{W_{1}(\tau) \otimes W_{c-1}(\tau)}\left({ }^{l_{1, c-1}} v\right)=\xi_{W_{1}(\tau) \otimes W_{c-1}(\tau)}\left({ }^{l_{1, c-1}} v x\right)=\psi\left(\operatorname{tr}\left(v_{i, j}^{3} x_{i, j}\right)\right) \xi_{W_{1}(\tau) \otimes W_{c-1}(\tau)}\left({ }^{l_{1, c-1}} v\right) .
$$

The first equality follows since $\xi$ is unramified, the second follows from the invariance properties of $W_{c-1}(\tau)$. Thus the coordinates of $v_{i, j}^{3}$ can be taken in $\mathcal{O}$, and since $\xi$ is unramified, the integration over these coordinates becomes an integral of the constant function 1 over $\operatorname{Mat}_{c-1 \times 1}(\mathcal{O})$. Since the measure of $\mathcal{O}$ was chosen to be 1 , this integration evaluates to the constant 1 . Proceeding with this argument for $(i, j)=(k-2, k-1),(k-3, k-2)$, etc., the blocks $v_{l, l+1}^{3}$ can each be taken in $\mathcal{O}$ and the integral over these coordinates is 1 , for $l=k-1, \ldots, 1$. Then we continue with $v_{k-2, k}^{3}$ using $\mathcal{X}_{k-2, k}$ and in this way show that all the diagonal $v_{l, l+2}^{3}$ can be taken in $\mathcal{O}$, 
$l=k-2, \ldots, 1$. The last block to consider is $v_{1, k}^{3}$, which we handle using $\mathcal{X}_{1, k}$. We deduce

$$
\begin{aligned}
\int_{V^{3}} \xi_{W_{1}(\tau) \otimes W_{c-1}(\tau)}\left(l^{\left.l_{1, c-1} v\right) d v}\right. & =1 \times \xi_{W_{1}(\tau) \otimes W_{c-1}(\tau)}\left(I_{k c}\right) \\
& =\int_{V^{4}} \int_{V^{1}} T_{l_{1, c-1}} \xi\left(\operatorname{diag}\left(w_{k, 1}{ }^{l_{1, c-1}} v^{1}, w_{k, c-1} l_{1, c-1} v^{4}\right)\right) \psi^{-1}\left(v^{1}\right) \psi^{-1}\left(v^{4}\right) d v^{1} d v^{4},
\end{aligned}
$$

where for the second equality we used (3.11), and $T_{l_{1, c-1}}$ is the intertwining operator given by (3.8). Since $\xi$ is unramified, $T_{l_{1, c-1}} \xi$ is a scalar multiple of the normalized unramified vector $\xi^{\prime}$ in the space of (3.9). We only need to show that this scalar is nonzero. We may decompose $T_{l_{1, c-1}}$ into rank-1 intertwining operators on spaces of the form

$$
\operatorname{Ind}_{B_{\mathrm{GL}_{2}}}^{\mathrm{GL}_{2}}\left(||^{-(c-2 l+1) / 2} \chi_{i} \otimes||^{-\left(c-2 l^{\prime}+1\right) / 2} \chi_{j}\right), \quad i<j, \quad l^{\prime} \leq l-1 .
$$

According to the Gindikin-Karpelevich formula ([Cas80, Theorem 3.1]), each intertwining operator takes the normalized unramified vector in this space to a constant multiple of the normalized unramified vector in its image, and this constant is given by

$$
\frac{1-q^{-1-l+l^{\prime}} \chi_{i}(\varpi) \chi_{j}^{-1}(\varpi)}{1-q^{-l+l^{\prime}} \chi_{i}(\varpi) \chi_{j}^{-1}(\varpi)} .
$$

Since $-l+l^{\prime} \leq-1$, if the quotient has a zero or pole, then $1-q^{-s} \chi_{i}(\varpi) \chi_{j}^{-1}(\varpi)=0$ for $\operatorname{Re}(s) \geq 1$, contradicting our assumption.

We deduce that

$$
\int_{V^{4}} \int_{V^{1}} T_{l_{1, c-1}} \xi\left(\operatorname{diag}\left(w_{k, 1}^{l_{1, c-1}} v^{1}, w_{k, c-1}^{l_{1, c-1}} v^{4}\right)\right) \psi^{-1}\left(v^{1}\right) \psi^{-1}\left(v^{4}\right) d v^{1} d v^{4}
$$

is a nonzero multiple of

$$
\int_{V^{4}} \int_{V^{1}} \xi^{\prime}\left(\operatorname{diag}\left(w_{k, 1}{ }^{l_{1, c-1}} v^{1}, w_{k, c-1}^{l_{1, c-1}} v^{4}\right)\right) \psi^{-1}\left(v^{1}\right) \psi^{-1}\left(v^{4}\right) d v^{1} d v^{4}
$$

Since $\xi^{\prime}\left(\operatorname{diag}\left(x, I_{k(c-1)}\right)\right)$ (resp., $\left.\xi^{\prime}\left(\operatorname{diag}\left(I_{k}, y\right)\right)\right)$ is the normalized unramified vector in the space of $\sigma_{k, 1}$ (resp., $\left.\sigma_{k, c-1}\right)$, and by the inductive hypothesis the $(k, 1)$ (resp., $\left.(k, c-1)\right)$ functional is nonzero on this element, we conclude that the $(k, c)$ functional is nonzero on $\xi$.

Recall the diagonal embedding $\mathrm{GL}_{c} \rightarrow \mathrm{GL}_{k c}$ given by $h \mapsto h^{\Delta}=\operatorname{diag}(h, \ldots, h)$. We prove a local analog of Claim 8.

Proposition 24. Let $\lambda$ be a $(k, c)$ functional on $\sigma_{k, c}$. For a vector $\xi$ in the space of $\sigma_{k, c}$, let $\lambda_{\xi}(g)=\lambda(g \cdot \xi)\left(g \in \mathrm{GL}_{k c}\right)$. Then $\lambda_{\xi}\left(h^{\Delta} g\right)=\tau\left((\operatorname{det} h) I_{k}\right) \lambda_{\xi}(g)$ for all $h \in \mathrm{GL}_{c}$. In particular $\lambda_{\xi}\left(h^{\Delta} g\right)=\lambda_{\xi}(g)$ for $h \in \mathrm{SL}_{c}$. The same assertion applies to the representation $\rho_{c}(\tau)$.

Proof. Since the representation $\sigma_{k, c}$ admits a unique $(k, c)$ functional, we can assume that $\lambda$ is given by (3.4). Since $h^{\Delta}$ normalizes $V_{\left(c^{k}\right)}$ without changing the measure, stabilizes $\psi$ and commutes with $w_{k, c}$,

$$
\lambda_{\xi}\left(h^{\Delta} g\right)=\int_{V_{\left(c^{k}\right)}} \xi\left(h^{\Delta} w_{k, c} v g\right) \psi^{-1}(v) d v .
$$

Now the assertion follows because $\xi\left(h^{\Delta} g\right)=\prod_{i} \chi_{i}(\operatorname{det}(h)) \xi(g)$ by the definition of $\sigma_{k, c}$. Since $W_{c}(\tau)$ is a summand of the $(k, c)$ model of $\sigma_{k, c}$, the same result is valid for $\rho_{c}(\tau)$. 
Remark 25. Since the space of $(k, c)$ functionals on $\sigma_{k, c}$ is one-dimensional, $J_{V_{\left(c^{k}\right)}, \psi}\left(\sigma_{k, c}\right)$ is one-dimensional. Hence a priori $\mathrm{GL}_{c}^{\Delta}$ acts by a character (which must then be trivial on $\mathrm{SL}_{c}^{\Delta}$ ).

Claim 26. Let $\tau$ be an unramified twist of an irreducible generic unramified and unitary representation of $\mathrm{GL}_{k}$. Then $\sigma_{k, c}$ is irreducible and the assumption of Corollary 23 is satisfied.

Proof. Let $d \in \mathbb{R}$ be such that $\tau=|\operatorname{det}|^{d} \tau_{0}$, where $\tau_{0}$ is an irreducible generic unramified and unitary representation of $\mathrm{GL}_{k}$. Write $\tau_{0}=\operatorname{Ind}_{B_{\mathrm{GL}_{k}}}^{\mathrm{GL}_{k}}\left(\chi_{1}^{0} \otimes \ldots \otimes \chi_{k}^{0}\right)$. As we explained in $\S 2.2$, the representation $\operatorname{Ind}_{P_{\left(c^{k}\right)}}^{\mathrm{GL}_{k c}}\left(\chi_{1}^{0} \otimes \ldots \otimes \chi_{k}^{0}\right)$ is irreducible and $q^{-1 / 2}<\left|\chi_{i}^{0}(\varpi)\right|<q^{1 / 2}$ for all $i$. Since $\chi_{i}=\mid{ }^{d} \chi_{i}^{0}$, we conclude that $\sigma_{k, c}$ is irreducible and $\left|\chi_{i}(\varpi) \chi_{j}^{-1}(\varpi)\right|=\left|\chi_{i}^{0}(\varpi)\left(\chi_{j}^{0}\right)^{-1}(\varpi)\right|<q$.

3.5. The computation of the integrals with unramified data. In this section we compute the integrals from (3.1) with unramified data. We use the notation and conventions from $\S 3.3$. Let $G=\mathrm{Sp}_{2 n}$ or $\mathrm{SO}_{2 n}$. Let $\pi$ be an irreducible unramified representation of $G$. Let $\tau$ be an unramified twist of an irreducible unitary generic unramified representation of $\mathrm{GL}_{k}$. Recall that the $G \times \mathrm{GL}_{k}$ integrals were described in $\S 3.1$ and the $\mathrm{GL}_{n} \times \mathrm{GL}_{k}$ integrals were defined in $\S 3.2$. We also use notation from these sections.

Define

$$
\begin{aligned}
d_{\tau}(s)= & {\left[\frac{L(\alpha s+1 / 2, \tau)}{L(\alpha s+n+1 / 2, \tau)}\right] \prod_{1 \leq j \leq\lfloor n / 2\rfloor} \frac{L\left(2 \alpha s+2 j, \tau, \mathrm{Sym}^{2}\right)}{L\left(2 \alpha s+2 j+2 n-2\lfloor n / 2\rfloor-1, \tau, \mathrm{Sym}^{2}\right)} } \\
& \times \prod_{1 \leq j \leq\lceil n / 2\rceil} \frac{L\left(2 \alpha s+2 j-1, \tau, \wedge^{2}\right)}{L\left(2 \alpha s+2 j+2 n-2\lceil n / 2\rceil, \tau, \wedge^{2}\right)},
\end{aligned}
$$

where if $G=\mathrm{Sp}_{2 n}, \alpha=2 k n+1$, and if $G=\mathrm{SO}_{2 n}, \alpha=2 k n-1$; and the factor in square brackets here and in Theorem 29 below is included only for $\mathrm{Sp}_{2 n}$.

Let $\omega^{0}$ be the unramified matrix coefficient of $\pi^{\vee}$ normalized such that $\omega^{0}\left(I_{2 n}\right)=1$. Let $f_{W_{c}(\tau)}^{0}$ be the unramified element in the space of $\operatorname{Ind}_{P}^{H}\left(W_{c}(\tau)\right)$ normalized by $f_{W_{c}(\tau)}^{0}\left(I_{2 k c}\right)=1$, and extended to a standard section of $\operatorname{Ind}_{P}^{H}\left(W_{c}(\tau) \delta_{P}^{s}\right)$.

The following lemma reduces the $G \times \mathrm{GL}_{k}$ integral to the $\mathrm{GL}_{n} \times \mathrm{GL}_{k}$ integral. Its proof occupies $\S 3.5 .1$ below.

Lemma 27. Assume $\pi$ is an irreducible quotient of $\operatorname{Ind}_{R}^{G}\left(\pi_{n}\right)$, where $R=M_{R} \ltimes U_{R}$ is the Siegel parabolic subgroup with $M_{R}=\left\{\operatorname{diag}\left(a, a^{*}\right): a \in \mathrm{GL}_{n}\right\}$ and $\pi_{n}$ is an irreducible unramified representation of $\mathrm{GL}_{n}$. Let $\omega_{n}^{0}$ be the normalized unramified matrix coefficient of $\pi_{n}^{\vee}$ and $\rho_{W_{n}(\tau) \otimes W_{n}\left(\tau^{\vee}\right)}^{0}$ be the normalized unramified function in the space of

$$
\operatorname{Ind}_{P_{(k n, k n)}}^{\mathrm{GL}_{2 k n}}\left(W_{n}(\tau) \otimes W_{n}\left(\tau^{\vee}\right)\right) .
$$

Then

$$
Z\left(s, \omega^{0}, f_{W_{c}(\tau)}^{0}\right)=d_{\tau}(s) Z\left(\alpha s /(k n), \omega_{n}^{0}, \rho_{W_{n}(\tau) \otimes W_{n}\left(\tau^{\vee}\right)}^{0}\right) .
$$

Since $\pi$ is irreducible and unramified, by Langlands' classification one can choose an unramified principal series representation $\pi_{n}^{\prime}$ of $\mathrm{GL}_{n}$, such that $\pi$ is a quotient of $\operatorname{Ind}_{R}^{G}\left(\pi_{n}^{\prime}\right)$ and in addition, $\pi_{n}^{\prime}$ contains an irreducible unramified quotient $\pi_{n}$. Then $\operatorname{Ind}_{R}^{G}\left(\pi_{n}\right)$ is an unramified quotient of $\operatorname{Ind}_{R}^{G}\left(\pi_{n}^{\prime}\right)$, hence contains $\pi$. Thus the assumption of the lemma is always satisfied.

Theorem 28. For irreducible unramified representations $\pi$ of $\mathrm{GL}_{n}$, $\tau$ and $\tau^{\prime}$ of $\mathrm{GL}_{k}$ (as in $\S$ 3.2), if $\omega^{0}$ is the normalized unramified matrix coefficient of $\pi^{\vee}$ and $f_{W_{n}(\tau) \otimes W_{n}\left(\tau^{\prime}\right)}^{0}$ is the 
normalized unramified element in the space of $\operatorname{Ind}_{P_{(k n, k n)}}^{\mathrm{GL}_{2 k n}}\left(W_{n}(\tau) \otimes W_{n}\left(\tau^{\prime}\right)\right)$,

$$
Z\left(s, \omega^{0}, f_{W_{n}(\tau) \otimes W_{n}\left(\tau^{\prime}\right)}^{0}\right)=\frac{L\left(k n s+1 / 2, \pi^{\vee} \times \tau\right) L\left(k n s+1 / 2, \pi \times \tau^{\prime \vee}\right)}{\prod_{j=1}^{n} L\left(2 k n s+j, \tau \times \tau^{\prime \vee}\right)} .
$$

This theorem is proved in $\S 3.6$. As a corollary we obtain the computation of the $G \times \mathrm{GL}_{k}$ integrals with unramified data.

Theorem 29. ${ }^{2}$ When all data are unramified,

$$
Z\left(s, \omega^{0}, f_{W_{c}(\tau)}^{0}\right)=\frac{L(\alpha s+1 / 2, \pi \times \tau)}{[L(\alpha s+n+1 / 2, \tau)] \prod_{1 \leq j \leq n} L\left(2 \alpha s+2 j, \tau, \wedge^{2}\right) L\left(2 \alpha s+2 j-1, \tau, \mathrm{Sym}^{2}\right)} .
$$

Proof of Theorem 29. According to Theorem 28 the $\mathrm{GL}_{n} \times \mathrm{GL}_{k}$ integral

$$
Z\left(\alpha s /(k n), \omega_{n}^{0}, \rho_{W_{n}(\tau) \otimes W_{n}\left(\tau^{\vee}\right)}^{0}\right)=\frac{L\left(\alpha s+1 / 2, \pi_{n}^{\vee} \times \tau\right) L\left(\alpha s+1 / 2, \pi_{n} \times \tau\right)}{\prod_{j=0}^{n-1} L(2 \alpha s+j+1, \tau \times \tau)} .
$$

Combining this with Lemma 27, the formula (3.12) for $d_{\tau}(s)$ and using the identities

$$
\begin{aligned}
& L(s, \pi \times \tau)=[L(s, \tau)] L\left(s, \pi_{n} \times \tau\right) L\left(s, \pi_{n}^{\vee} \times \tau\right), \\
& L(s, \tau \times \tau)=L\left(s, \tau, \operatorname{Sym}^{2}\right) L\left(s, \tau, \wedge^{2}\right)
\end{aligned}
$$

(see $\S 3.3)$ gives the result.

Now we can deduce the meromorphic continuation of the global partial $L$-function.

Theorem 30. Let $\pi$ and $\tau$ be irreducible automorphic cuspidal representations of $G(\mathbb{A})$ and $\mathrm{GL}_{k}(\mathbb{A})$, respectively. Let $S$ be a finite set of places of $F$, outside which all data are unramified. Then $L^{S}(s, \pi \times \tau)$ admits meromorphic continuation to $\mathbb{C}$.

Remark 31. This theorem is not new, it follows from Langlands' general theory of Eisenstein series [Lan67, Lan76], which is applicable in a much wider setting (e.g., for a large class of groups $G$ ). It is provided as an illustration of the applicability of our results.

Proof. According to Theorem 1, the global integral $Z\left(s, \varphi_{1}, \varphi_{2}, f\right)$ admits meromorphic continuation to $\mathbb{C}$, and for $\operatorname{Re}(s) \gg 0$ coincides with $(2.1)$. For decomposable data, we can write (2.1) in the form (3.1): the product of an integral $Z_{S}$ and infinitely many local integrals $Z_{\nu}$ for the places $\nu \notin S$. The integral $Z_{S}$ is meromorphic and can be chosen to be holomorphic and nonzero, in a neighborhood of a given $s \in \mathbb{C}$. This can be proved along the lines of Theorem 21 (which deals with one place). Therefore the product of integrals over the places outside $S$ admits meromorphic continuation.

For each integral $Z_{\nu}$ with $\nu \notin S$, all data are unramified: the local representations $\pi_{\nu}$ and $\tau_{\nu}$ are irreducible unramified, $\tau_{\nu}$ is also generic, and $\psi_{\nu}$ is unramified. In addition, because $\tau=|\operatorname{det}|{ }^{d} \tau_{0}$ for some $d \in \mathbb{R}$ where $\tau_{0}$ is unitary, $\tau_{\nu}$ is the unramified twist of a unitary representation.

By virtue of Theorem 29 (applied to $Z_{\nu}$ ), the product of local integrals over all $\nu \notin S$ is precisely $L^{S}(s, \pi \times \tau)$ divided by products of partial $L$-functions $L^{S}(s, \tau), L^{S}\left(s, \tau, \wedge^{2}\right)$ and $L^{S}\left(s, \tau, \mathrm{Sym}^{2}\right)$ (with $s$ replaced by a suitable linear polynomial of $s$ ). Since by Langlands' general theory of Eisenstein series [Lan67, Lan76], each of the $L$-functions in the denominator is meromorphic, we deduce that $L^{S}(s, \pi \times \tau)$ admits meromorphic continuation.

\footnotetext{
${ }^{2}$ There was a typo in the formula in the original announcement [CFGK]; we would like to thank Dihua Jiang for pointing it out to us.
} 
Remark 32. In a subsequent paper ([CFK]) we develop the local theory of the doubling integrals over all places of $F$ (including the ramified and archimedean ones), and define the local $\gamma$-, $\epsilon$ - and $L$-factors. This enables us to define the complete $L$-function $L(s, \pi \times \tau)$, and study its analytic behavior. In particular we show that it satisfies a global functional equation $L(s, \pi \times \tau)=$ $\epsilon(s, \pi \times \tau) L\left(1-s, \pi^{\vee} \times \tau^{\vee}\right)$.

3.5.1. Proof of Lemma 2\%. The proof consists of two steps. First, we use the realization of the $(k, c)$ functional using $(k, a)$ and $(k, b)$ functionals given in $\S 3.4$, for $a=b=n$. Note that here $c=2 n$. This changes the inducing data of $f_{W_{c}(\tau)}$. Then we write the unipotent integration over $U_{0}$ as an iterated integral, where the inner part is "almost" an intertwining operator (some coordinates are missing, they are taken from $U_{R}$ ), the middle part is the unipotent integration of a $\mathrm{GL}_{n} \times \mathrm{GL}_{k}$ integral, and the outer integral reduces to a constant. This essentially completes the reduction, with the remaining part being to compute the proportionality factor $d_{\tau}(s)$ of the operator.

We replace the matrix coefficient with a suitable element of an unramified principal series. Since $\pi$ is an irreducible quotient of $\operatorname{Ind}_{R}^{G}\left(\pi_{n}\right)$, the representation $\pi^{\vee}$ is a subrepresentation of $\operatorname{Ind}_{R}^{G}\left(\pi_{n}^{\vee}\right)$, and we can further regard $\pi_{n}^{\vee}$ as a subrepresentation of an unramified principal series representation of $\mathrm{GL}_{n}$. By transitivity of induction, $\pi^{\vee}$ is embedded in an unramified principal series of $G$. Specifically, this is obtained by taking a function $\phi^{\vee}$ in the space of $\operatorname{Ind}_{R}^{G}\left(\pi_{n}^{\vee}\right)$ and evaluating at the identity of $G$. Thus we can realize the $G$-invariant pairing on $\pi \times \pi^{\vee}$ using the Iwasawa decomposition $G=B_{G} K_{G}$. Let $\phi^{0}$ and $\phi^{\vee, 0}$ be the unramified vectors in the spaces of $\operatorname{Ind}_{R}^{G}\left(\pi_{n}\right)$ and $\operatorname{Ind}_{R}^{G}\left(\pi_{n}^{\vee}\right)$, respectively, normalized by $\phi^{0}\left(I_{2 n}\right)=\phi^{\vee, 0}\left(I_{2 n}\right)=1$. Then

$$
\omega^{0}(g)=\int_{K_{G}} \phi^{0}(o) \phi^{\vee 0}(o g) d o=\int_{K_{G}} \phi^{\vee, 0}(o g) d o .
$$

Observe that for any $g_{0} \in G$,

$$
\begin{aligned}
& \int_{U_{0}} f_{W_{c}(\tau)}^{0}\left(\delta u_{0}\left(g_{0},{ }^{\iota} g_{0}\right)\left(1,{ }^{\iota} g\right), s\right) \psi_{U}\left(u_{0}\right) d u_{0} \\
& =\int_{U_{0}} f_{W_{c}(\tau)}^{0}\left(\operatorname{diag}\left(g_{0}, \ldots, g_{0}, g_{0}^{*}, \ldots, g_{0}^{*}\right) \delta u_{0}\left(1,{ }^{\iota} g\right), s\right) \psi_{U}\left(u_{0}\right) d u_{0}
\end{aligned}
$$

(direct computation) and by Proposition 24, for any $h \in H$,

$$
f_{W_{c}(\tau)}^{0}\left(\operatorname{diag}\left(g_{0}, \ldots, g_{0}, g_{0}^{*}, \ldots, g_{0}^{*}\right) h, s\right)=f_{W_{c}(\tau)}^{0}(h, s) .
$$

In addition, the embeddings of the two copies of $G$ in $H$ commute and $f_{W_{c}(\tau)}^{0}$ is right $K_{H^{-}}$ invariant, so that for any $o \in K_{G}$,

$$
f_{W_{c}(\tau)}^{0}\left(h\left(1,{ }^{\iota}\left(o^{-1} g\right)\right), s\right)=f_{W_{c}(\tau)}^{0}\left(h\left(1,{ }^{\iota}\left(o^{-1} g\right)\right)\left(o^{-1}, 1\right), s\right)=f_{W_{c}(\tau)}^{0}\left(h\left(o^{-1},{ }^{\iota} o^{-1}\right)\left(1,{ }^{\iota} g\right), s\right) .
$$

Therefore

$$
\begin{aligned}
Z\left(s, \omega^{0}, f_{W_{c}(\tau)}^{0}\right) & =\int_{G}\left(\int_{K_{G}} \phi^{\vee, 0}(o g) d o\right) \int_{U_{0}} f_{W_{c}(\tau)}^{0}\left(\delta u_{0}\left(1,{ }^{\iota} g\right), s\right) \psi_{U}\left(u_{0}\right) d u_{0} d g \\
& =\int_{G} \int_{K_{G}} \phi^{\vee, 0}(g) \int_{U_{0}} f_{W_{c}(\tau)}^{0}\left(\delta u_{0}\left(1,{ }^{\iota}\left(o^{-1} g\right)\right), s\right) \psi_{U}\left(u_{0}\right) d u_{0} d o d g \\
& =\int_{G} \phi^{\vee, 0}(g) \int_{U_{0}} f_{W_{c}(\tau)}^{0}\left(\delta u_{0}\left(1,{ }^{\iota} g\right), s\right) \psi_{U}\left(u_{0}\right) d u_{0} d g .
\end{aligned}
$$


Note that the measure of $K_{G}$ was taken to be 1 . Apply Lemma 22 to the function on $\mathrm{GL}_{k c}$ given by $x \mapsto f_{W_{c}(\tau)}\left(\operatorname{diag}\left(x, x^{*}\right) h, s\right)$ with $a=b=n$. Then with $V^{3}$ and $l_{n, n}$ as defined in $\S 3.4$,

$$
f_{W_{c}(\tau)}^{0}(h, s)=\int_{V^{3}} f_{W_{n}(\tau) \otimes W_{n}(\tau)}^{0}\left(l_{n, n} v h, s\right) d v .
$$

Using transitivity of induction and (3.6), we see that $f_{W_{n}(\tau) \otimes W_{n}(\tau)}^{0}(h, s)$ belongs to the space of the representation

$$
\operatorname{Ind}_{L}^{H}\left(|\operatorname{det}|^{-n / 2+\alpha s} W_{n}(\tau) \otimes|\operatorname{det}|^{n / 2+\alpha s} W_{n}(\tau)\right),
$$

where $L$ is the standard parabolic subgroup of $H$ with a Levi part $M_{L}=\mathrm{GL}_{k n} \times \mathrm{GL}_{k n}$. It is an unramified function. In addition, $f_{W_{n}(\tau) \otimes W_{n}(\tau)}^{0}\left(I_{2 k c}, s\right)=1$ because by Lemma 22 , if we assume that $W_{c}(\tau)$ is realized by $(3.4)$,

$$
1=f_{W_{c}(\tau)}^{0}\left(I_{2 k c}, s\right)=\int_{V^{3}} f_{W_{n}(\tau) \otimes W_{n}(\tau)}^{0}\left({ }^{l_{n, n}} v, s\right)=f_{W_{n}(\tau) \otimes W_{n}(\tau)}^{0}\left(I_{2 k c}, s\right),
$$

where for the last equality see the proof of Corollary 23 (and recall that the volume of $\mathcal{O}$ is 1 ).

With the above modifications, integral (3.14) becomes

$$
\int_{G} \phi^{\vee, 0}(g) \int_{U_{0}} \int_{V^{3}} f_{W_{n}(\tau) \otimes W_{n}(\tau)}^{0}\left(l_{n, n} v \delta u_{0}\left(1,{ }^{\iota} g\right), s\right) \psi_{U}\left(u_{0}\right) d v d u_{0} d g .
$$

This integral is absolutely convergent for $\operatorname{Re}(s) \gg 0$ as a triple integral; this is obtained using the auxiliary complex parameters which guarantee the convergence of (3.4) (if $\zeta_{1} \gg \ldots \gg \zeta_{k} \gg$ 0 , then $\operatorname{Re}(s) \gg \zeta_{1}$, see e.g., [Sou00, Lemma 3.1], [Kap13c, Claim 5.20]). All forthcoming manipulations are justified in this right half-plane.

Next we shift $v$ to the right of $\left(1,{ }^{\iota} \mathrm{g}\right)$. Observe the following properties, which are immediate to verify.

(1) $\delta_{0}$ normalizes $V^{3}$.

(2) For $v \in V^{3},{ }^{v} \delta_{1}=\delta_{1} u^{\prime}$ where $u^{\prime} \in U_{0}$ and $\psi_{U}\left(u^{\prime}\right)=1$.

(3) The elements of $V^{3}$ normalize $U_{0}$ and fix $\left.\psi_{U}\right|_{U_{0}}$.

(4) $V^{3}$ commutes with $\left(1,{ }^{\iota} g\right)$.

(5) $\delta_{0}$ commutes with $l_{n, n}$.

(6) $l_{n, n}$ commutes with $\left(1,{ }^{\iota} g\right)$.

$\left(\delta_{0}, \delta_{1}\right.$ were given in $\S 3.1$.) We also see that

$$
U_{0}^{\prime}={ }^{l_{n, n}} U_{0}=\left\{\left(\begin{array}{cccc}
I_{k n} & & U_{1} & U_{2} \\
& I_{k n} & U_{3} & U_{4} \\
& & I_{k n} & \\
& & & I_{k n}
\end{array}\right)\right\},
$$

where $U_{1}=\left(\begin{array}{cc}* \\ 0 & *\end{array}\right)$ with $0 \in$ Mat $_{n}$, so that

$$
\left\{\left(\begin{array}{cc}
I_{k n} & U_{1} \\
& I_{k n}
\end{array}\right)\right\}
$$

is the unipotent subgroup appearing in the integral for $\mathrm{GL}_{n} \times \mathrm{GL}_{k}$ (defined in $\S 3.2$ ); for $\mathrm{Sp}_{2 n}$ (resp., $\mathrm{SO}_{2 n}$ ), restriction of $\psi_{U}$ to the coordinates of $U_{1}$ gives the character $\psi_{U}$ (resp., $\psi_{U}^{-1}$ ) for the $\mathrm{GL}_{n} \times \mathrm{GL}_{k}$ integral; $\psi_{U}$ is trivial on $U_{2}$ and $U_{3} ; U_{2}$ and $U_{3}$ each takes the form $\left(\begin{array}{c}* \\ 0\end{array} *\right.$ * $)$ where $0 \in$ Mat $_{n}$; and $U_{4}$ is already determined by $U_{1}$ and the form defining $H$. 
Utilizing properties (1)-(6), integral (3.16) equals

$$
\int_{G} \phi^{\vee, 0}(g) \int_{U_{0}^{\prime}} \int_{V^{3}} f_{W_{n}(\tau) \otimes W_{n}(\tau)}^{0}\left(\delta_{0}\left({ }^{l_{n, n}} \delta_{1}\right) u_{0}^{\prime}\left(1,{ }^{\iota} g\right) l_{n, n} v, s\right) \psi_{U}\left(u_{0}^{\prime}\right) d v d u_{0}^{\prime} d g .
$$

Here $\psi_{U}$ is regarded as a character of $U_{0}^{\prime}$ using conjugation $\left({ }^{l_{n, n}^{-1}} u_{0}^{\prime} \in U_{0}\right)$.

To produce a $\mathrm{GL}_{n} \times \mathrm{GL}_{k}$ integral (pertaining to the statement of the lemma), we must alter $f_{W_{n}(\tau) \otimes W_{n}(\tau)}^{0}$ such that its restriction to $\mathrm{GL}_{2 k n} \cong M_{P}$ becomes a section of a representation induced from $W_{n}(\tau) \otimes W_{n}\left(\tau^{\vee}\right)$. This would be the result of an application of an intertwining operator. If we had arbitrary coordinates in the bottom left $n \times n$ block of $U_{2}$, then the integral over $U_{2}$ together with the Weyl element $\operatorname{diag}\left(I_{k n},\left({ }_{\epsilon_{0} I_{k n}} I_{k n}\right), I_{k n}\right)$ (when $G=\mathrm{Sp}_{2 n}$ or $k n$ is even) would constitute this operator (recall that $\epsilon_{0}=-1$ for $\mathrm{Sp}_{2 n}$ and $\epsilon_{0}=1$ for $\mathrm{SO}_{2 n}$ ). To fill in these missing coordinates we factor the integral through $U_{R}$. Refer to $\S 3.2$ for the definition of the $\mathrm{GL}_{n} \times \mathrm{GL}_{k}$ integral.

Let $U_{0}^{\bullet}$ be the group obtained from $U_{0}^{\prime}$ by replacing the 0 block of $U_{2}$ with arbitrary coordinates. This group will "receive" the coordinates from $U_{R}$. We can still write the elements of $U_{0}^{\bullet}$ in the form (3.17), i.e.,

$$
\left\{\left(\begin{array}{cccc}
I_{k n} & & U_{1} & U_{2} \\
& I_{k n} & U_{3} & U_{4} \\
& & I_{k n} & \\
& & & I_{k n}
\end{array}\right)\right\},
$$

the only difference being the block $U_{2}$, which now does not contain the 0 block. Now factoring (3.19) through $U_{R}$, it becomes

$$
\int_{U_{R} \backslash G} \int_{U_{R}} \phi^{\vee, 0}(z g) \int_{U_{0}^{\prime}} \int_{V^{3}} f_{W_{n}(\tau) \otimes W_{n}(\tau)}^{0}\left(\delta_{0}\left({ }^{l n, n} \delta_{1}\right) u_{0}^{\prime}\left(1,{ }^{\iota}(z g)\right) l_{n, n} v, s\right) \psi_{U}\left(u_{0}^{\prime}\right) d v d u_{0}^{\prime} d z d g .
$$

By definition $\phi^{\vee, 0}(z g)=\phi^{\vee, 0}(g)$. For $z \in U_{R}$ and $u_{0}^{\prime} \in U_{0}^{\prime}$,

$$
\left(1,{ }^{\iota} z^{-1}\right)\left(\left({ }^{l_{n, n}} \delta_{1}\right) u_{0}^{\prime}\right)=m_{z}\left({ }^{l_{n, n}} \delta_{1}\right) u_{z},
$$

where $m_{z}$ belongs to the unipotent subgroup $V_{\left((2 n)^{k}\right)}$ of $M_{P}$ and $u_{z} \in U_{0}$. Moreover, as $z$ and $u_{0}^{\prime}$ vary over $U_{R}$ and $U_{0}^{\prime}, u_{z}$ varies over $U_{0}^{\bullet}$. In coordinates, put $z=\left(\begin{array}{cc}I_{n} & z \\ & I_{n}\end{array}\right)$ and for $1 \leq l \leq 4$ and $1 \leq i, j \leq k$, denote the $(i, j)$-th block of $U_{l}$ appearing in (3.20) by $U_{l}^{i, j} \in$ Mat $_{n}$. For $u_{0}^{\prime}$, the block corresponding to $U_{l}^{i, j}$ is denoted by $\left[u_{0}^{\prime}\right]_{l}^{i, j}$. Then $m_{z} \in V_{((2 k-1) n, n)},{ }^{\delta_{0}} m_{z} \in V_{(n,(2 k-1) n)}$ and the top $n$ rows of ${ }^{\delta_{0}} m_{z}$ are

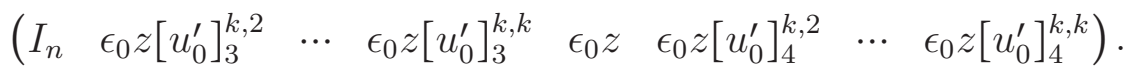

We change variables in $u_{z}$ to remove the dependency on $z$. The change is described as follows: for $l \in\{1,3\}, 1 \leq i \leq k-1$ and $2 \leq j \leq k$,

$$
\begin{array}{ll}
{\left[u_{0}^{\prime}\right]_{l}^{i, j} \mapsto\left[u_{0}^{\prime}\right]_{l}^{i, j}-\epsilon_{0}\left[u_{0}^{\prime}\right]_{l}^{i, 1} z\left[u_{0}^{\prime}\right]_{3}^{k, j},} & {\left[u_{0}^{\prime}\right]_{1}^{k, j} \mapsto\left[u_{0}^{\prime}\right]_{1}^{k, j}+z\left[u_{0}^{\prime}\right]_{3}^{k, j},} \\
{\left[u_{0}^{\prime}\right]_{2}^{i, j} \mapsto\left[u_{0}^{\prime}\right]_{2}^{i, j}-\epsilon_{0}\left[u_{0}^{\prime}\right]_{1}^{i, 1} z\left[u_{0}^{\prime}\right]_{4}^{k, j},} & {\left[u_{0}^{\prime}\right]_{2}^{i, 1} \mapsto\left[u_{0}^{\prime}\right]_{2}^{i, 1}-\epsilon_{0}\left[u_{0}^{\prime}\right]_{1}^{i, 1} z}
\end{array}
$$

In this list, changes to $\left[u_{0}^{\prime}\right]_{2}^{i, j}$ and $\left[u_{0}^{\prime}\right]_{3}^{i, j}$ are only applied if $i+j \leq k+1$ for $\operatorname{Sp}_{2 n}$ and $i+j \leq k$ for $\mathrm{SO}_{2 n}$, because outside of this range the coordinates are already determined by the definition of $H$. Only the change to $\left[u_{0}^{\prime}\right]_{1}^{k, 2}$ affects $\psi_{U}$, and we obtain $\psi\left(\operatorname{tr}\left(-\epsilon_{0} z\left[u_{0}^{\prime}\right]_{3}^{k, 2}\right)\right)$ (for $\mathrm{SO}_{2 n}$ as 
mentioned after $(3.18) \psi_{U}$ restricts to the inverse of the character for the $\mathrm{GL}_{n} \times \mathrm{GL}_{k}$ integral, i.e., to $\left.\psi^{-1}\left(\operatorname{tr}\left(U_{1}^{k, 2}\right)\right)\right)$. In addition, for any $h \in H$,

$$
f_{W_{n}(\tau) \otimes W_{n}(\tau)}^{0}\left(m_{z} h, s\right)=\psi\left(\operatorname{tr}\left(\epsilon_{0} z\left[u_{0}^{\prime}\right]_{3}^{k, 2}\right)\right) f_{W_{n}(\tau) \otimes W_{n}(\tau)}^{0}(h, s)
$$

because of the character of the (top left) $(k, n)$-functional $W_{n}(\tau)$. Therefore $(3.21)$ becomes

$$
\int_{U_{R} \backslash G} \phi^{\vee, 0}(g) \int_{U_{0}^{\bullet}} \int_{V^{3}} f_{W_{n}(\tau) \otimes W_{n}(\tau)}^{0}\left(\delta_{0}\left({ }^{l_{n, n}} \delta_{1}\right) u_{0}^{\bullet}\left(1,{ }^{\iota} g\right) l_{n, n} v, s\right) \psi_{U}\left(u_{0}^{\bullet}\right) d v d u_{0}^{\bullet} d g .
$$

Note that here the integration over $U_{R}$ is incorporated into the integration over $U_{0}^{\bullet}$.

Write $\delta_{0}=w \delta_{0}^{\prime} w^{\prime}$ as follows. For $G=\mathrm{Sp}_{2 n}, \delta_{0}^{\prime}$ is the embedding in $M_{P}$ of the element $\left({ }_{I_{k n}} I_{k n}\right)$ corresponding to $\delta_{0}$ in the $\mathrm{GL}_{n} \times \mathrm{GL}_{k}$ integral, and

$$
w=w^{\prime}=\left(\begin{array}{llll}
I_{k n} & & & \\
& & I_{k n} & \\
& -I_{k n} & & \\
& & & I_{k n}
\end{array}\right) \text {. }
$$

If $G=\mathrm{SO}_{2 n}$, set $\kappa=I_{4 k n}$ if $k n$ is even, otherwise $\kappa=\operatorname{diag}\left(I_{2 k n-1},\left({ }_{1}{ }^{1}\right), I_{2 k n-1}\right)$. Then $\delta_{0}^{\prime}$ is the embedding of $\left({ }_{I_{k n}} I_{k n}\right)$ in $M_{\kappa}$, i.e., when $k n$ is odd, it is obtained from the embedding in $M_{P}$ by conjugation with $\kappa$, and

$$
w=\left(\begin{array}{cccc}
I_{k n} & & & \\
& & I_{k n} & \\
& I_{k n} & & \\
& & & I_{k n}
\end{array}\right) \kappa, \quad w^{\prime}=\kappa\left(\begin{array}{llll}
I_{k n} & & & \\
& & I_{k n} & \\
& I_{k n} & & \\
& & & I_{k n}
\end{array}\right) .
$$

(The element $\kappa$ is needed because when $k n$ is odd, we must have $\operatorname{det} w=\operatorname{det} w^{\prime}=1$.) To make the notation uniform, set $\kappa=I_{4 k n}$ when $G=\mathrm{Sp}_{2 n}$.

For $u_{0}^{\bullet} \in U_{0}^{\bullet}$, let $u^{i}$ denote the element obtained from $u_{0}^{\bullet}$ by zeroing out the coordinates in the blocks $U_{j}$ with $j \neq i$ (see $\left.(3.20)\right)$. Write

$$
\delta_{0} u_{0}^{\bullet}=w \cdot{ }^{\left(\delta_{0}^{\prime} w^{\prime}\right)} u^{2} \cdot \delta_{0}^{\prime} \cdot w^{\prime}\left(u^{1} u^{4}\right) \cdot w^{\prime} u^{3} .
$$

Since ${ }^{l_{n, n}} \delta_{1} \in U_{P}$, it commutes with $u_{0}^{\bullet}$ and with $u^{3}$. Also $\delta_{1}^{\prime}=w^{\prime}\left(l_{n, n} \delta_{1}\right)$ and $\delta^{\prime}=\delta_{0}^{\prime} \delta_{1}^{\prime}$ are the embeddings in $M_{\kappa} P$ of the elements corresponding to $\delta_{1}$ and $\delta$ for the $\mathrm{GL}_{n} \times \mathrm{GL}_{k}$ integral, except that for $G=\mathrm{SO}_{2 n}, \delta_{1}^{\prime}$ is actually the embedding of $\delta_{1}^{-1}$, and $\delta^{\prime}$ is the embedding of

$$
\left(\begin{array}{c}
I_{k n} \\
I_{k n}
\end{array}\right) \operatorname{diag}\left(I_{(k-1) n},\left(\begin{array}{cc}
I_{n} & -I_{n} \\
I_{n}
\end{array}\right), I_{(k-1) n}\right) .
$$

Then

$$
\delta_{0}\left({ }^{l_{n, n}} \delta_{1}\right) u_{0}^{\bullet}=w \cdot{ }^{\left(\delta_{0}^{\prime} w^{\prime}\right)} u^{2} \cdot \delta^{\prime} \cdot w^{\prime}\left(u^{1} u^{4}\right) \cdot w^{\prime} u^{3} .
$$

Denote the subgroup of elements ${ }^{\left(\delta_{0}^{\prime} w^{\prime}\right)} u^{2}$ by $U^{2}$, let $U^{1,4}$ be the subgroup of elements $w^{\prime}\left(u^{1} u^{4}\right)$ and $U^{3}$ be the subgroup of elements $u^{3}$. For example,

$$
U^{2}=\left\{\left(\begin{array}{cccc}
I_{k n} & & & \\
& I_{k n} & Z & \\
& & I_{k n} & \\
& & & I_{k n}
\end{array}\right) \in H\right\} .
$$


Recall that $u^{4}$ is uniquely determined by $u^{1}$ and $H$. Then for any $h \in H$,

$$
\begin{aligned}
& \int_{U_{0}^{\bullet}} f_{W_{n}(\tau) \otimes W_{n}(\tau)}^{0}\left(\delta_{0}\left({ }^{l_{n, n}} \delta_{1}\right) u_{0}^{\bullet} h, s\right) \psi_{U}\left(u_{0}^{\bullet}\right) d u_{0}^{\bullet} \\
& =\int_{U^{3}} \int_{U^{1,4}} \int_{U^{2}} f_{W_{n}(\tau) \otimes W_{n}(\tau)}^{0}\left(w u^{2} \delta^{\prime} u w^{\prime} u^{3} h, s\right) \psi_{U}(u) d u^{2} d u d u^{3} .
\end{aligned}
$$

Below we will show that the integration over $U^{3}$ evaluates to the constant 1 . The $d u$-integral is the unipotent integration appearing in the $\mathrm{GL}_{n} \times \mathrm{GL}_{k}$ integral (defined in $\S 3.2$ ), when we identify $\mathrm{GL}_{2 k n}$ with $M_{\kappa} P$. The integration over $U^{2}$ defines an intertwining operator $M(s)$ from the space of (3.15) to

$$
\operatorname{Ind}_{\kappa_{L}}^{H}\left(|\operatorname{det}|^{-n / 2+\alpha s} W_{n}(\tau) \otimes|\operatorname{det}|^{-n / 2-\alpha s} W_{n}\left(\tau^{\vee}\right)\right)
$$

The image $M(s) f_{W_{n}(\tau) \otimes W_{n}(\tau)}^{0}$ of $M(s)$ on $f_{W_{n}(\tau) \otimes W_{n}(\tau)}^{0}$ is the normalized unramified vector multiplied by a constant which we denote $d_{\tau}(s)$, and we indeed prove below that it is equal to (3.12). Again, identify $\mathrm{GL}_{2 k n}$ with $M_{\kappa} P$. When we restrict $M(s) f_{W_{n}(\tau) \otimes W_{n}(\tau)}^{0}$ to $\mathrm{GL}_{2 k n}$ we obtain a rational section of

$$
|\operatorname{det}|^{(\alpha-n) / 2} \operatorname{Ind}_{P_{(k n, k n)}}^{\mathrm{GL}_{2 k n}}\left(\left(W_{n}(\tau) \otimes W_{n}\left(\tau^{\vee}\right)\right) \delta_{P_{(k n, k n)}}^{\ell s}\right), \quad \ell=\alpha /(k n) .
$$

Let $\rho_{W_{n}(\tau) \otimes W_{n}\left(\tau^{\vee}\right)}^{0}$ be the normalized unramified vector in the space of

$$
\operatorname{Ind}_{P_{(k n, k n)}}^{\mathrm{GL}_{2 k n}}\left(W_{n}(\tau) \otimes W_{n}\left(\tau^{\vee}\right)\right) .
$$

Then for any $h \in \mathrm{GL}_{2 k n}$,

$$
M(s) f_{W_{n}(\tau) \otimes W_{n}(\tau)}^{0}(h, s)=|\operatorname{det} h|^{(\alpha-n) / 2} d_{\tau}(s) \rho_{W_{n}(\tau) \otimes W_{n}\left(\tau^{\vee}\right)}^{0}(h, \ell s),
$$

where $h \mapsto \rho_{W_{n}(\tau) \otimes W_{n}\left(\tau^{\vee}\right)}^{0}(h, \ell s)$ is the standard section of

$$
\operatorname{Ind}_{P_{(k n, k n)}}^{\mathrm{GL}_{2 k n}}\left(\left(W_{n}(\tau) \otimes W_{n}\left(\tau^{\vee}\right)\right) \delta_{P_{(k n, k n)}}^{\ell s}\right)
$$

corresponding to $\rho_{W_{n}(\tau) \otimes W_{n}\left(\tau^{\vee}\right)}^{0}$. Now (3.22) takes the form

$$
\int_{U_{R} \backslash G} \phi^{\vee, 0}(g) \int_{V^{3}} \int_{U^{3}} \int_{U^{1,4}} M(s) f_{W_{n}(\tau) \otimes W_{n}(\tau)}^{0}\left(\delta^{\prime} u w^{\prime} u^{3}\left(1,{ }^{\iota} g\right) l_{n, n} v, s\right) \psi_{U}(u) d u d u^{3} d v d g .
$$

Let $g=\operatorname{diag}\left(g^{\prime}, g^{\prime *}\right) \in M_{R}, g^{\prime} \in \mathrm{GL}_{n}$. Then

$$
w^{\prime}\left(1,{ }^{\iota} g\right)=\operatorname{diag}\left(I_{k n}, g^{\prime}, I_{(k-1) n}\right) \in \mathrm{GL}_{2 k n}
$$

is the embedding $\left(I_{n}, \mathrm{GL}_{n}\right)$ in the construction of the $\mathrm{GL}_{n} \times \mathrm{GL}_{k}$ integral. Apply the Iwasawa decomposition $G=R K_{G}$. The change of measure $\delta_{R}^{-1}(g)$ incurred by this decomposition, the conjugation of $U^{3}$ by $\left(1,{ }^{\iota} g\right)$ and the additional $\delta_{R}^{1 / 2}(g)$ emitted from $\phi^{\vee, 0}$, multiply the integrand by $\left|\operatorname{det} g^{\prime}\right|^{(n-\alpha) / 2}$ (which will cancel out with the power of $|\operatorname{det}|$ from (3.23)). Also note that $\phi^{\vee, 0}(g)=\delta_{R}^{1 / 2}(g) \phi_{n}^{\vee, 0}\left(g^{\prime}\right)$, where $\phi_{n}^{\vee, 0}$ is the normalized unramified vector in the space of $\pi_{n}^{\vee}$. Then (3.25) equals

$$
\int_{V^{3}} \int_{U^{3}} \int_{\mathrm{GL}_{n}} \int_{U^{1,4}}\left|\operatorname{det} g^{\prime}\right|^{(n-\alpha) / 2} \phi_{n}^{\vee, 0}\left(g^{\prime}\right) M(s) f_{W_{n}(\tau) \otimes W_{n}(\tau)}^{0}\left(\delta^{\prime} u\left(1, g^{\prime}\right) w^{\prime} u^{3} l_{n, n} v, s\right) \psi_{U}(u) d u d g^{\prime} d u^{3} d v \text {. }
$$


Let $\phi_{n}^{0}$ be the normalized unramified vector in the space of $\pi_{n}$. Since for $g^{\prime} \in \mathrm{GL}_{n}$,

$$
\omega_{n}^{0}\left(g^{\prime}\right)=\int_{K_{\mathrm{GL}_{n}}} \phi_{n}^{0}(o) \phi_{n}^{\vee, 0}\left(o g^{\prime}\right) d o=\int_{K_{\mathrm{GL}_{n}}} \phi_{n}^{\vee, 0}\left(o g^{\prime}\right) d o,
$$

as in the beginning of this section we can replace $\phi_{n}^{\vee, 0}\left(g^{\prime}\right)$ with $\omega_{n}^{0}\left(g^{\prime}\right)$ (see $\S 3.6 .1$ for more details). Then (3.26) becomes

$$
\int_{V^{3}} \int_{U^{3}} \int_{\mathrm{GL}_{n}} \int_{U^{1,4}}\left|\operatorname{det} g^{\prime}\right|^{(n-\alpha) / 2} \omega_{n}^{0}\left(g^{\prime}\right) M(s) f_{W_{n}(\tau) \otimes W_{n}(\tau)}^{0}\left(\delta^{\prime} u\left(1, g^{\prime}\right) w^{\prime} u^{3} l_{n, n} v, s\right) \psi_{U}(u) d u d g^{\prime} d u^{3} d v
$$

$$
=\int_{V^{3}} \int_{U^{3}} Z^{\prime}\left(|\operatorname{det}|^{(n-\alpha) / 2} \omega_{n}^{0},\left(w^{\prime} u^{3} l_{n, n} v\right) \cdot M(s) f_{W_{n}(\tau) \otimes W_{n}(\tau)}^{0}\right) d u^{3} d v
$$

where $Z^{\prime}(\cdots)$ is the $\mathrm{GL}_{n} \times \mathrm{GL}_{k}$ integral, with the exception that for $\mathrm{SO}_{2 n}, \delta_{1}^{\prime}\left(\delta_{1}^{\prime}\right.$ is the unipotent part of $\delta^{\prime}$ ) and $\psi_{U}$ appearing in $Z^{\prime}$ are the inverses of those defined in $\S 3.2$. The value of the $\mathrm{GL}_{n} \times \mathrm{GL}_{k}$ integral with unramified data is invariant with respect to this change. To see this, replace the section on $\mathrm{GL}_{2 k n}$ with its right translate by $\operatorname{diag}\left(-I_{k n}, I_{k n}\right)$ (this matrix commutes with the embedding $\left.\left(\mathrm{GL}_{n}, \mathrm{GL}_{n}\right)<\mathrm{GL}_{2 k n}\right)$.

We will show that the $d u^{3}$-integral in (3.27) vanishes unless $v \in K_{H}$ and $u^{3} \in K_{H}$. Since $M(s) f_{W_{n}(\tau) \otimes W_{n}(\tau)}^{0}$ is unramified, for any $v, u^{3} \in K_{H}$ and $h \in H$ we have

$$
M(s) f_{W_{n}(\tau) \otimes W_{n}(\tau)}^{0}\left(h\left(w^{\prime} u^{3} l_{n, n} v\right), s\right)=M(s) f_{W_{n}(\tau) \otimes W_{n}(\tau)}^{0}(h, s),
$$

thus (3.27) becomes

$Z^{\prime}\left(|\operatorname{det}|^{(n-\alpha) / 2} \omega_{n}^{0}, M(s) f_{W_{n}(\tau) \otimes W_{n}(\tau)}^{0}\right) \int_{V^{3}} \int_{U^{3}} 1 d u^{3} d v=1 \times Z^{\prime}\left(|\operatorname{det}|^{(n-\alpha) / 2} \omega_{n}^{0}, M(s) f_{W_{n}(\tau) \otimes W_{n}(\tau)}^{0}\right)$,

because the measure of $\mathcal{O}$ is 1 . Finally using (3.24),

$$
Z^{\prime}\left(|\operatorname{det}|^{(n-\alpha) / 2} \omega_{n}^{0}, M(s) f_{W_{n}(\tau) \otimes W_{n}(\tau)}^{0}\right)=d_{\tau}(s) Z\left(\ell s, \omega_{n}^{0}, \rho_{W_{n}(\tau) \otimes W_{n}\left(\tau^{\vee}\right)}^{0}\right),
$$

which is the integral appearing on the right hand side of (3.13). This will complete the proof of the lemma, once we handle the integrals over $V^{3}$ and $U^{3}$ in (3.27) and compute $d_{\tau}(s)$.

Conjugate the elements $w^{\prime}$ and $l_{n, n}$ to the right. They disappear because $M(s) f_{W_{n}(\tau) \otimes W_{n}(\tau)}^{0}$ is $K_{H}$-invariant on the right. In coordinates, for $G=\mathrm{Sp}_{2 n}$,

$$
w^{\prime}\left(U^{3} \cdot{ }_{n, n} V^{3}\right)=\left\{\left(\begin{array}{cccc}
I_{k n} & & & \\
& I_{k n} & & \\
V & U^{3} & I_{k n} & \\
0 & V^{\prime} & & I_{k n}
\end{array}\right) \in H\right\}, \quad V=\left(\begin{array}{cccc}
0 & V_{1,2}^{3} & \cdots & V_{1, k}^{3} \\
\vdots & & \ddots & \vdots \\
\vdots & & & V_{k-1, k}^{3} \\
0 & \cdots & \cdots & 0
\end{array}\right), \quad V_{i, j}^{3} \in \text { Mat }_{n},
$$

where $V^{\prime}$ is uniquely defined given $V$ and $H$.

To show that the coordinates of $V_{i, j}^{3}$ must belong to $\mathcal{O}$, otherwise the $d u^{3}$-integral vanishes, consider matrices

$$
x=\left(\begin{array}{cccc}
I_{k n} & & {[x]} & \\
& I_{k n} & & {[x]^{\prime}} \\
& & I_{k n} & \\
& & & I_{k n}
\end{array}\right) \in H, \quad[x]=\left(\begin{array}{cccc}
x_{1,2} & 0 & \cdots & 0 \\
\vdots & \ddots & \ddots & \vdots \\
x_{1, k} & \cdots & x_{k-1, k} & 0 \\
0 & \cdots & \cdots & 0
\end{array}\right), \quad x_{i, j} \in \text { Mat }_{n} .
$$

For each $1 \leq i \leq k-1$ and $2 \leq j \leq k$, let $\mathcal{X}_{i, j}$ be the subgroup of these matrices $x$ where the only nonzero block in $[x]$ is $x_{i, j}$, which takes arbitrary coordinates in $\mathcal{O}$. Then $\mathcal{X}_{i, j}<K_{H}$. We handle 
$V_{i, j}^{3}$ using $\mathcal{X}_{i, j}$. Starting with $V_{1,2}^{3}$, we proceed along the diagonal $(l, l+1)$ with $l=2, \ldots, k-1$ in increasing order, then the diagonal $(l, l+2), l=1, \ldots, k-2$, etc., the last block of $V$ to handle being $V_{1, k}^{3}$, for which we use $\mathcal{X}_{1, k}$.

Consider $z \in w^{\prime}\left(U^{3} \cdot l_{n, n} V^{3}\right)$. Let $v_{i, j}^{3}$ be the block of $z$ corresponding to $V_{i, j}^{3}$. If $v_{i, j}^{3} \in \operatorname{Mat}_{n}(\mathcal{O})$, we can assume $v_{i, j}^{3}=0$ since $M(s) f_{W_{n}(\tau) \otimes W_{n}(\tau)}^{0}$ is $K_{H^{-}}$-invariant on the right. To show $v_{i, j}^{3} \epsilon$ $\operatorname{Mat}_{n}(\mathcal{O})$, assuming we have already shown this for the previous blocks in the order along the diagonals, note that for $x \in \mathcal{X}_{i, j},{ }^{-1} z=u_{x} z_{x}$ with $u_{x} \in P$ and $z_{x} \in w^{\prime}\left(U^{3} \cdot{ }^{l_{n, n}} V^{3}\right)$. The projection of $u_{x}$ to $M_{P}$ belongs to the unipotent group $U$ of the $\mathrm{GL}_{n} \times \mathrm{GL}_{k}$ integral. The invariance properties of this integral (see (3.3)) imply that

$$
\begin{aligned}
& Z^{\prime}\left(|\operatorname{det}|^{(n-\alpha) / 2} \omega_{n}^{0},(z x) \cdot M(s) f_{W_{n}(\tau) \otimes W_{n}(\tau)}^{0}\right) \\
& =\psi\left(\operatorname{tr}\left(v_{i, j}^{3} x_{i, j}\right)\right) Z^{\prime}\left(|\operatorname{det}|^{(n-\alpha) / 2} \omega_{n}^{0}, z_{x} \cdot M(s) f_{W_{n}(\tau) \otimes W_{n}(\tau)}^{0}\right) .
\end{aligned}
$$

Regarding $z_{x}$, the coordinates depending on $x$ belong to the blocks of $U^{3}$ and this dependence can be removed by a change of variables. Therefore if we consider the integral $d u^{3}$ over the coordinates of $U^{3}$ appearing in $z$,

$$
\begin{aligned}
& \int_{U^{3}} Z^{\prime}\left(|\operatorname{det}|^{(n-\alpha) / 2} \omega_{n}^{0}, z \cdot M(s) f_{W_{n}(\tau) \otimes W_{n}(\tau)}^{0}\right) d u^{3} \\
& =\int_{U^{3}} Z^{\prime}\left(|\operatorname{det}|^{(n-\alpha) / 2} \omega_{n}^{0},(z x) \cdot M(s) f_{W_{n}(\tau) \otimes W_{n}(\tau)}^{0}\right) d u^{3} \\
& =\psi\left(\operatorname{tr}\left(v_{i, j}^{3} x_{i, j}\right)\right) \int_{U^{3}} Z^{\prime}\left(|\operatorname{det}|^{(n-\alpha) / 2} \omega_{n}^{0}, z \cdot M(s) f_{W_{n}(\tau) \otimes W_{n}(\tau)}^{0}\right) d u^{3} .
\end{aligned}
$$

Hence the integral $d u^{3}$ is zero unless $v_{i, j}^{3} \in \operatorname{Mat}_{n}(\mathcal{O})$ (cf. the proof of Corollary 23), and we can proceed to the next block.

Next we handle the coordinates of $U^{3}$. Let $U_{i, j}$ denote the $(i, j)$-th $n \times n$ block of $U^{3}(1 \leq$ $i, j \leq k)$ and note that $U_{k, 1}$ is 0 , because this is the bottom left block of $U^{3}$ (see after (3.18)). We show that the coordinates of $U_{i, j}$ can be taken in $\mathcal{O}$. Consider

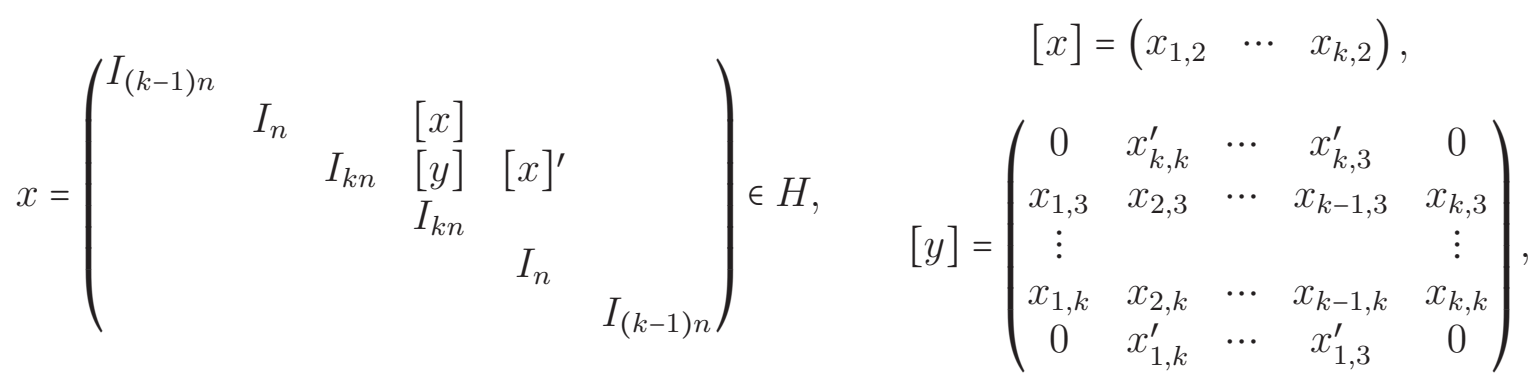

where $x_{i, j} \in$ Mat $_{n}$. Note that while $[x]$ can take arbitrary coordinates, in the notation for $[y]$ coordinates are dependent, since $x \in H$. Let $\mathcal{X}_{i, j}$ be the subgroup of matrices $x$ such that $x_{i, j} \in \operatorname{Mat}_{n}(\mathcal{O})$ (if $j>2, x_{i, j}$ also depends on $H$ ) and all other blocks which are independent of $x_{i, j}$ are 0 . We handle $U_{i, j}$ using $\mathcal{X}_{i, j}$ and as above, the order matters.

Let $z \in w^{\prime} U^{3}$. Denote the coordinates in $z$ corresponding to the blocks $U_{i, j}$ by $u_{i, j}$. For any $i$ and $j$, if $u_{i, j} \in \operatorname{Mat}_{n}(\mathcal{O})$, we can assume $u_{i, j}=0$ because the section is right $K_{H^{-}}$-invariant. Let $1 \leq i \leq k$. If $u_{l_{1}, 1}=u_{l_{2}, 2}=0$ for all $l_{1} \geq i$ and $l_{2}>i$, then for $x \in \mathcal{X}_{i, 2}$ we have $x^{-1} z=u_{x} z$, where 
$u_{x} \in M_{P}, u_{x}$ belongs to the unipotent subgroup $U_{0}$ appearing in the $\mathrm{GL}_{n} \times \mathrm{GL}_{k}$ integral and

$$
\begin{aligned}
& Z^{\prime}\left(|\operatorname{det}|^{(n-\alpha) / 2} \omega_{n}^{0}, z \cdot M(s) f_{W_{n}(\tau) \otimes W_{n}(\tau)}^{0}\right) \\
& =Z^{\prime}\left(|\operatorname{det}|^{(n-\alpha) / 2} \omega_{n}^{0},(z x) \cdot M(s) f_{W_{n}(\tau) \otimes W_{n}(\tau)}^{0}\right) \\
& =\psi\left(\operatorname{tr}\left(x_{i, 2} u_{i, 2}\right)\right) Z^{\prime}\left(|\operatorname{det}|^{(n-\alpha) / 2} \omega_{n}^{0}, z \cdot M(s) f_{W_{n}(\tau) \otimes W_{n}(\tau)}^{0}\right) .
\end{aligned}
$$

Thus the integrand vanishes unless $u_{i, 2} \in \operatorname{Mat}_{n}(\mathcal{O})$. We start with $U_{k, 2}$ using $\mathcal{X}_{k, 2}$ and deduce $U_{k, 2} \subset \operatorname{Mat}_{n}(\mathcal{O})$, which implies $U_{k-1,1} \subset \operatorname{Mat}_{n}(\mathcal{O})$. Then $U_{k-1,2} \subset \operatorname{Mat}_{n}(\mathcal{O})$, using $\mathcal{X}_{k-1,2}$.

To handle $U_{k, 3}$ a change of variables is needed. For $x \in \mathcal{X}_{k, 3},{ }^{-1} z=u_{x} z_{x}$, where $z_{x}$ belongs to ${ }^{w^{\prime}} U^{3}$ but depends on $x$. However, we can change variables in the blocks $U_{i, j}$ with $i \leq k-2$ and $j \geq 3$ (in particular, blocks which have not been handled) to remove this dependency. The element $u_{x}$ belongs to $P$ and its projection to $M_{P}$ is in the subgroup $U$ of the $\mathrm{GL}_{n} \times \mathrm{GL}_{k}$ integral. It follows that

$$
\begin{aligned}
& \int_{U^{3}} Z^{\prime}\left(|\operatorname{det}|^{(n-\alpha) / 2} \omega_{n}^{0}, z \cdot M(s) f_{W_{n}(\tau) \otimes W_{n}(\tau)}^{0}\right) d u^{3} \\
& =\int_{U^{3}} \int_{\mathcal{X}_{k, 3}} Z^{\prime}\left(|\operatorname{det}|^{(n-\alpha) / 2} \omega_{n}^{0},(z x) \cdot M(s) f_{W_{n}(\tau) \otimes W_{n}(\tau)}^{0}\right) d x d u^{3} \\
& =\int_{U^{3}} Z^{\prime}\left(|\operatorname{det}|^{(n-\alpha) / 2} \omega_{n}^{0}, z \cdot M(s) f_{W_{n}(\tau) \otimes W_{n}(\tau)}^{0}\right) d u^{3} \int_{\mathcal{X}_{k, 3}} \psi\left(\operatorname{tr}\left(x_{k, 3} u_{k, 3}\right)\right) d x,
\end{aligned}
$$

which equals zero unless $u_{k, 3} \in \operatorname{Mat}_{n}(\mathcal{O})$ (the measure of $\mathcal{X}_{k, 3}$ was taken to be 1 ). Thus $U_{k, 3} \subset$ $\operatorname{Mat}_{n}(\mathcal{O})$ and then also $U_{k-2,1} \subset \operatorname{Mat}_{n}(\mathcal{O})$, so that we can proceed with $U_{k-2,2}$. In general, once $U_{i, 2} \subset \operatorname{Mat}_{n}(\mathcal{O})$, we handle the diagonal $U_{i+l, 2+l}, l=k-i, \ldots, 1$. Then in particular $U_{i-1,1} \subset \operatorname{Mat}_{n}(\mathcal{O})$ so that we can continue with $U_{i-1,2}$. Once $U_{1,2} \subset \operatorname{Mat}_{n}(\mathcal{O})$ (thereby $U_{k-1, k} \subset$ $\operatorname{Mat}_{n}(\mathcal{O})$ ), we proceed with the remaining blocks $U_{i, j}$ on the diagonals, from bottom to top: the first diagonal is $U_{l, k}, U_{l-1, k-1}, \ldots, U_{1, k-l+1}$ with $l=k-1$, the next diagonal $U_{l, k}, \ldots, U_{1, k-l+1}$ with $l=k-2$, etc. The last block is $U_{1, k}$. In this way, the changes of variables are always to blocks which have not been considered.

The case of $\mathrm{SO}_{2 n}$ is similar: $V^{3}$ is the same, there are fewer coordinates in $U^{3}$.

It remains to compute $d_{\tau}(s)$. Consider the standard Levi subgroup of $H$ isomorphic to $\mathrm{GL}_{k n} \times H^{\prime}$, where $H^{\prime}$ is a classical group of the same type as $H$ but with rank $k n$. Regard $H^{\prime}$ as a subgroup of $H$ via this isomorphism. Fix the Borel subgroup $B_{H^{\prime}}=B_{H} \cap H^{\prime}$. Looking at (3.15) we see that the restriction of $f_{W_{n}(\tau) \otimes W_{n}(\tau)}^{0}$ to $H^{\prime}$ is the normalized unramified element, in the space of the unramified principal series representation of $H^{\prime}$ induced (normalized induction) from

$$
\otimes_{1 \leq i \leq k, 1 \leq j \leq n} \chi_{i}||^{\alpha s+j-1 / 2} .
$$

Let $\Delta(\tau, n, s)$ be the irreducible unramified constituent of this representation.

Assume $H^{\prime}=\mathrm{Sp}_{2 k n}$. The subgroup $U^{2}$ is the unipotent radical of a standard parabolic subgroup of $H^{\prime}$ whose Levi part is $\mathrm{GL}_{k n}$. The adjoint action of $\mathrm{GL}_{k n}(\mathbb{C})$ on the Lie algebra of the $L$-group of $U^{2}$ is st $\oplus \wedge^{2}$, where st is the standard representation. By Langlands' theory [Lan67] and the Gindikin-Karpelevich formula [Cas80, Theorem 3.1],

$$
d_{\tau}(s)=\frac{L(0, \Delta(\tau, n, s), \text { st })}{L(1, \Delta(\tau, n, s), \text { st })} \frac{L\left(0, \Delta(\tau, n, s), \wedge^{2}\right)}{L\left(1, \Delta(\tau, n, s), \wedge^{2}\right)} .
$$


The first quotient (for the standard representation) contributes

$$
\prod_{1 \leq i \leq k} \prod_{1 \leq j \leq n} \frac{L\left(\alpha s+j-1 / 2, \chi_{i}\right)}{L\left(\alpha s+j+1 / 2, \chi_{i}\right)}=\frac{L(\alpha s+1 / 2, \tau)}{L(\alpha s+n+1 / 2, \tau)} .
$$

The second quotient (the exterior square) contributes, for each pair $1 \leq i \neq i^{\prime} \leq k$,

$$
\prod_{1 \leq j, j^{\prime} \leq n} \frac{L\left(2 \alpha s+j+j^{\prime}-1, \chi_{i} \chi_{i^{\prime}}\right)}{L\left(2 \alpha s+j+j^{\prime}, \chi_{i} \chi_{i^{\prime}}\right)}=\prod_{1 \leq j \leq n} \frac{L\left(2 \alpha s+j, \chi_{i} \chi_{i^{\prime}}\right)}{L\left(2 \alpha s+j+n, \chi_{i} \chi_{i^{\prime}}\right)},
$$

and for $1 \leq i \leq k$,

$$
\prod_{1 \leq j_{1}<n} \prod_{j_{1}<j_{2} \leq n} \frac{L\left(2 \alpha s+j_{1}+j_{2}-1, \chi_{i}^{2}\right)}{L\left(2 \alpha s+j_{1}+j_{2}, \chi_{i}^{2}\right)}=\prod_{1 \leq j<n} \frac{L\left(2 \alpha s+2 j, \chi_{i}^{2}\right)}{L\left(2 \alpha s+j+n, \chi_{i}^{2}\right)} .
$$

(This product is empty for $n=1$, then it simply equals 1.) Thus when $n$ is odd we obtain

$$
\prod_{1 \leq j \leq(n-1) / 2} \frac{L\left(2 \alpha s+2 j, \tau, \mathrm{Sym}^{2}\right)}{L\left(2 \alpha s+2 j+n, \tau, \mathrm{Sym}^{2}\right)} \prod_{1 \leq j \leq(n+1) / 2} \frac{L\left(2 \alpha s+2 j-1, \tau, \wedge^{2}\right)}{L\left(2 \alpha s+2 j+n-1, \tau, \wedge^{2}\right)},
$$

and for even $n$,

$$
\prod_{1 \leq j \leq n / 2} \frac{L\left(2 \alpha s+2 j, \tau, \mathrm{Sym}^{2}\right)}{L\left(2 \alpha s+2 j+n-1, \tau, \mathrm{Sym}^{2}\right)} \prod_{1 \leq j \leq n / 2} \frac{L\left(2 \alpha s+2 j-1, \tau, \wedge^{2}\right)}{L\left(2 \alpha s+2 j+n, \tau, \wedge^{2}\right)} .
$$

The constant $d_{\tau}(s)$ is this product multiplied by (3.28). For $H^{\prime}=\mathrm{SO}_{2 k n}$ the adjoint action of $\mathrm{GL}_{k n}(\mathbb{C})$ on the Lie algebra of the dual group of $U^{2}$ is $\wedge^{2}$. The only change in the computation above is we omit (3.28). The proof of the lemma is complete.

3.6. Local factors for $\mathrm{GL}_{n}$. In this section we prove Theorem 28. We proceed with the set-up from $\S 3.3$. Let $G=\mathrm{GL}_{n}$ and $\pi$ be an irreducible unramified representation of $G$. Let $\tau$ and $\tau^{\prime}$ be unramified twists of irreducible unitary generic unramified representations of $\mathrm{GL}_{k}$, with the additional assumption on the central characters of $\tau$ and $\tau^{\prime}$, namely $\tau \tau^{\prime}\left(a I_{k}\right)=1$ for all $a \in F^{*}$. For the definition of the $\mathrm{GL}_{n} \times \mathrm{GL}_{k}$ integral see $\S 3.2$, but we recall that $H=\mathrm{GL}_{2 k n}$, $P=P_{(k n, k n)}$ and the sections belong to $\operatorname{Ind}_{P}^{H}\left(\left(W_{n}(\tau) \otimes W_{n}\left(\tau^{\prime}\right)\right) \delta_{P}^{s}\right)$.

Let $\omega^{0}$ and $f_{W_{n}(\tau) \otimes W_{n}\left(\tau^{\prime}\right)}^{0}$ be the normalized unramified elements given by the theorem. We reduce the $G \times \mathrm{GL}_{k}$ integral to the case $n=1$, which is computed directly. Put $\alpha=k n$ and for any positive integers $a$ and $b$ such that $a+b=n$,

$$
d_{\tau, \tau^{\prime}, a, b}(s)=\prod_{1 \leq j \leq b} \frac{L\left(2 \alpha s+j, \tau \times \tau^{\prime \vee}\right)}{L\left(2 \alpha s+a+j, \tau \times \tau^{\prime \vee}\right)} .
$$

Lemma 33. For $a$ and $b$ as above, write $\pi$ as a quotient of $\operatorname{Ind}_{R}^{G}\left(\pi_{a} \otimes \pi_{b}\right)$, where $R=P_{(a, b)}$ and $\pi_{a}$ and $\pi_{b}$ are irreducible unramified representations of $\mathrm{GL}_{a}$ and $\mathrm{GL}_{b}$. Let $\omega_{a}^{0}$ and $\omega_{b}^{0}$ be the normalized unramified matrix coefficients of $\pi_{a}^{\vee}$ and $\pi_{b}^{\vee}, \rho_{W_{a}(\tau) \otimes W_{a}\left(\tau^{\prime}\right)}^{0}$ be the normalized unramified function in the space of

$$
\operatorname{Ind}_{P_{(k a, k a)}}^{\mathrm{GL}_{2 k a}}\left(W_{a}(\tau) \otimes W_{a}\left(\tau^{\prime}\right)\right)
$$

and $\varrho_{W_{b}(\tau) \otimes W_{b}\left(\tau^{\prime}\right)}^{0}$ be the normalized unramified function in the space of

$$
\operatorname{Ind}_{P_{(k b, k b)}}^{\mathrm{GL}_{2 k b}}\left(W_{b}(\tau) \otimes W_{b}\left(\tau^{\prime}\right)\right) .
$$

Then

$$
Z\left(s, \omega^{0}, f_{W_{n}(\tau) \otimes W_{n}\left(\tau^{\prime}\right)}^{0}\right)=d_{\tau, \tau^{\prime}, a, b}(s) Z\left(\alpha s /(k a), \omega_{a}^{0}, \rho_{W_{a}(\tau) \otimes W_{a}\left(\tau^{\prime}\right)}^{0}\right) Z\left(\alpha s /(k b), \omega_{b}^{0}, \varrho_{W_{b}(\tau) \otimes W_{b}\left(\tau^{\prime}\right)}^{0}\right) .
$$


Proposition 34. For $n=1$ and when all data are unramified,

$$
Z\left(s, \omega^{0}, f_{W_{1}(\tau) \otimes W_{1}\left(\tau^{\prime}\right)}^{0}\right)=\frac{L\left(k s+1 / 2, \pi^{-1} \times \tau\right) L\left(k s+1 / 2, \pi \times \tau^{\prime \vee}\right)}{L\left(2 k s+1, \tau \times \tau^{\prime \vee}\right)} .
$$

The lemma is proved in $\S 3.6 .1$ and the proposition in $\S 3.7$. Now we can compute the integral inductively, for all $n$.

Proof of Theorem 28. We argue using induction on $n$. We have to show that the integral with unramified data equals

$$
\frac{L\left(k n s+1 / 2, \pi^{\vee} \times \tau\right) L\left(k n s+1 / 2, \pi \times \tau^{\prime \vee}\right)}{\prod_{j=1}^{n} L\left(2 k n s+j, \tau \times \tau^{\prime \vee}\right)} .
$$

The result holds for $n=1$ by Proposition 34. Consider a $\mathrm{GL}_{n} \times \mathrm{GL}_{k}$ integral. Assume the formula is true for $n-1$ and apply Lemma 33 to the integral with $a=1$ and $b=n-1$. The integral becomes the product of $d_{\tau, \tau^{\prime}, 1, n-1}(s)$, the $\mathrm{GL}_{1} \times \mathrm{GL}_{k}$ integral and the $\mathrm{GL}_{n-1} \times \mathrm{GL}_{k}$ integral. Using the case $n=1$,

$$
Z\left(n s, \omega_{1}^{0}, \rho_{W_{1}(\tau) \otimes W_{1}\left(\tau^{\prime}\right)}^{0}\right)=\frac{L\left(k n s+1 / 2, \pi_{1}^{-1} \times \tau\right) L\left(k n s+1 / 2, \pi_{1} \times \tau^{\prime \vee}\right)}{L\left(2 k n s+1, \tau \times \tau^{\prime \vee}\right)} .
$$

Applying the induction hypothesis to the $\mathrm{GL}_{n-1} \times \mathrm{GL}_{k}$ integral,

$$
Z\left(n s /(n-1), \omega_{n-1}^{0}, \varrho_{W_{n-1}(\tau) \otimes W_{n-1}\left(\tau^{\prime}\right)}^{0}\right)=\frac{L\left(k n s+1 / 2, \pi_{n-1}^{\vee} \times \tau\right) L\left(k n s+1 / 2, \pi_{n-1} \times \tau^{\prime \vee}\right)}{\prod_{j=1}^{n-1} L\left(2 k n s+j, \tau \times \tau^{\prime \vee}\right)} .
$$

Together we obtain

$$
\frac{L\left(k n s+1 / 2, \pi^{\vee} \times \tau\right) L\left(k n s+1 / 2, \pi \times \tau^{\prime \vee}\right)}{L\left(2 k n s+1, \tau \times \tau^{\prime \vee}\right)^{2} \prod_{j=2}^{n-1} L\left(2 k n s+j, \tau \times \tau^{\prime \vee}\right)}
$$

and multiplying this by

$$
d_{\tau, \tau^{\prime}, 1, n-1}(s)=\prod_{1 \leq j \leq n-1} \frac{L\left(2 k n s+j, \tau \times \tau^{\prime \vee}\right)}{L\left(2 k n s+1+j, \tau \times{\tau^{\prime}}^{\vee}\right)}=\frac{L\left(2 k n s+1, \tau \times \tau^{\prime \vee}\right)}{L\left(2 k n s+n, \tau \times \tau^{\prime \vee}\right)}
$$

gives the result.

We turn to the proof of the reduction lemma.

3.6.1. Proof of Lemma 33. The proof is a straightforward modification of the proof of Lemma 27, but manipulations applied to the $(k, c)$ functional are now doubled, because we work with both $W_{n}(\tau)$ and $W_{n}\left(\tau^{\prime}\right)$. We focus on the differences between the proofs, and when possible, use similar notation. Also recall that the definitions of $U_{0}, \delta, \delta_{0}, \delta_{1}$ and $\psi_{U}$ were given in $\S 3.2$.

We replace $\omega^{0}$ with the normalized unramified vector $\phi^{\vee, 0}$ in the space of $\operatorname{Ind}_{R}^{G}\left(\pi^{\vee}\right)$. As in the proof of Lemma 27, we write

$$
\omega^{0}(g)=\int_{K_{G}} \phi^{\vee, 0}(o g) d o .
$$


Then for any $g_{0} \in G$,

$$
\begin{aligned}
& \int_{U_{0}} f_{W_{n}(\tau) \otimes W_{n}\left(\tau^{\prime}\right)}^{0}\left(\delta u_{0}\left(g_{0}, g_{0}\right)(1, g), s\right) \psi_{U}\left(u_{0}\right) d u_{0} \\
& =\int_{U_{0}} f_{W_{n}(\tau) \otimes W_{n}\left(\tau^{\prime}\right)}^{0}\left(\operatorname{diag}\left(g_{0}, \ldots, g_{0}\right) \delta u_{0}(1, g), s\right) \psi_{U}\left(u_{0}\right) d u_{0} \\
& =\tau\left(\operatorname{det} g_{0} I_{k}\right) \tau^{\prime}\left(\operatorname{det} g_{0} I_{k}\right) \int_{U_{0}} f_{W_{n}(\tau) \otimes W_{n}\left(\tau^{\prime}\right)}^{0}\left(\delta u_{0}(1, g), s\right) \psi_{U}\left(u_{0}\right) d u_{0} .
\end{aligned}
$$

Here the second equality follows from Proposition 24. Our condition on the central characters of $\tau$ and $\tau^{\prime}$ implies $\tau\left(\operatorname{det} g_{0} I_{k}\right) \tau^{\prime}\left(\operatorname{det} g_{0} I_{k}\right)=1$. Combining this with the right $K_{H^{-}}$-invariance of $f_{W_{n}(\tau) \otimes W_{n}\left(\tau^{\prime}\right)}^{0}$, we deduce

$$
\begin{aligned}
Z\left(s, \omega^{0}, f_{W_{n}(\tau) \otimes W_{n}\left(\tau^{\prime}\right)}^{0}\right) & =\int_{G}\left(\int_{K_{G}} \phi^{\vee, 0}(o g) d o\right) \int_{U_{0}} f_{W_{n}(\tau) \otimes W_{n}\left(\tau^{\prime}\right)}^{0}\left(\delta u_{0}(1, g), s\right) \psi_{U}\left(u_{0}\right) d u_{0} d g \\
& =\int_{G} \phi^{\vee, 0}(g) \int_{U_{0}} f_{W_{n}(\tau) \otimes W_{n}\left(\tau^{\prime}\right)}^{0}\left(\delta u_{0}(1, g), s\right) \psi_{U}\left(u_{0}\right) d u_{0} d g .
\end{aligned}
$$

(Cf. (3.14).)

Let $a, b \geq 1$ be given by the statement of the lemma ( $a$ and $b$ need not be equal). Apply Lemma 22 twice, to the functions on $\mathrm{GL}_{k n}$ given by

$$
x \mapsto f_{W_{n}(\tau) \otimes W_{n}\left(\tau^{\prime}\right)}^{0}\left(\operatorname{diag}\left(x, I_{k n}\right) h, s\right), \quad y \mapsto f_{W_{n}(\tau) \otimes W_{n}\left(\tau^{\prime}\right)}^{0}\left(\operatorname{diag}\left(I_{k n}, y\right) h, s\right),
$$

where $h \in H$ is fixed. In the notation of that lemma,

$$
f_{W_{n}(\tau) \otimes W_{n}\left(\tau^{\prime}\right)}^{0}(h, s)=\int_{V^{3}} \int_{V^{3}} f_{\left(W_{a}(\tau) \otimes W_{b}(\tau)\right) \otimes\left(W_{a}\left(\tau^{\prime}\right) \otimes W_{b}\left(\tau^{\prime}\right)\right)}^{0}\left(\operatorname{diag}\left(l_{a, b}, l_{a, b}\right) \operatorname{diag}\left(v, v^{\prime}\right) h, s\right) d v d v^{\prime} .
$$

This is a section in the space of the representation

$$
\operatorname{Ind}_{L}^{H}\left(|\operatorname{det}|^{-b / 2+\alpha s} W_{a}(\tau) \otimes|\operatorname{det}|^{a / 2+\alpha s} W_{b}(\tau) \otimes|\operatorname{det}|^{-b / 2-\alpha s} W_{a}\left(\tau^{\prime}\right) \otimes|\operatorname{det}|^{a / 2-\alpha s} W_{b}\left(\tau^{\prime}\right)\right),
$$

where $L=P_{(k a, k b, k a, k b)}$.

Substituting (3.30) into (3.29) one obtains

$$
\int_{G} \phi^{\vee, 0}(g) \int_{U_{0}} \int_{V^{3}} \int_{V^{3}} f_{\ldots}^{0}\left(\operatorname{diag}\left(l_{a, b}, l_{a, b}\right) \operatorname{diag}\left(v, v^{\prime}\right) \delta u_{0}(1, g), s\right) \psi_{U}\left(u_{0}\right) d v d v^{\prime} d u_{0} d g .
$$

(Cf. (3.16).)

Properties (1)-(6) from $\S 3.5 .1$ now take the following form:

(1) $\delta_{0}^{-1} \operatorname{diag}\left(v, v^{\prime}\right)=\operatorname{diag}\left(v^{\prime}, v\right)$.

(2) If $v, v^{\prime} \in V^{3}, \operatorname{diag}\left(v^{\prime}, v\right) \delta_{1}=\delta_{1} u^{\prime}$ where $u^{\prime} \in U_{0}$ and $\psi_{U}\left(u^{\prime}\right)=1$.

(3) The elements of both copies of $V^{3}$ normalize $U_{0}$ and fix $\left.\psi_{U}\right|_{U_{0}}$.

(4) The group $\operatorname{diag}\left(V^{3}, I_{k n}\right)$ commutes with $(1, g)$.

(5) $\delta_{0}$ commutes with $\operatorname{diag}\left(l_{a, b}, l_{a, b}\right)$.

(6) $\operatorname{diag}\left(l_{a, b}, I_{k n}\right)$ commutes with $(1, g)$. 
Define

$$
U_{0}^{\prime}=\operatorname{diag}\left(l_{a, b}, l_{a, b}\right) U_{0}=\left\{\left(\begin{array}{cccc}
I_{k a} & & U_{1} & U_{2} \\
& I_{k b} & U_{3} & U_{4} \\
& & I_{k a} & \\
& & & I_{k b}
\end{array}\right)\right\} .
$$

Here $U_{4}$ is independent of $U_{1}$, so that

$$
\left\{\left(\begin{array}{cc}
I_{k a} & U_{1} \\
& I_{k a}
\end{array}\right)\right\}, \quad\left\{\left(\begin{array}{ll}
I_{k b} & U_{4} \\
& I_{k b}
\end{array}\right)\right\}
$$

are the unipotent subgroups corresponding to the $\mathrm{GL}_{a} \times \mathrm{GL}_{k}$ and $\mathrm{GL}_{b} \times \mathrm{GL}_{k}$ integrals, and restriction of $\psi_{U}$ to the coordinates of $U_{1}$ and $U_{4}$ gives the character $\psi_{U}$ defined for these integrals. The subgroup $U^{1,4}$ is now $U_{1} \times U_{4}$, and here we avoid the notation $U^{1,4}$. Also $\psi_{U}$ is

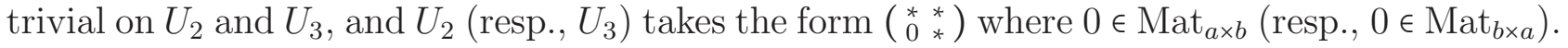
Cf. (3.17) and (3.18).

Utilizing properties (1)-(6), integral (3.32) equals

$$
\begin{aligned}
& \int_{G} \phi^{\vee, 0}(g) \int_{U_{0}^{\prime}} \int_{V^{3}} \int_{V^{3}} f_{\ldots}^{0}\left(\delta_{0}\left(\operatorname{diag}\left(l_{a, b}, l_{a, b}\right) \delta_{1}\right) u_{0}^{\prime} \operatorname{diag}\left(I_{k n}, l_{a, b} v\right)(1, g) \operatorname{diag}\left(l_{a, b} v^{\prime}, I_{k n}\right), s\right) \\
& \psi_{U}\left(u_{0}^{\prime}\right) d v d v^{\prime} d u_{0}^{\prime} d g .
\end{aligned}
$$

(Cf. (3.19).)

Let

$$
U_{0}^{\bullet}=\left\{\left(\begin{array}{cccc}
I_{k a} & & U_{1} & U_{2} \\
& I_{k b} & U_{3} & U_{4} \\
& & I_{k a} & \\
& & & I_{k b}
\end{array}\right)\right\},
$$

which is similar to (3.33) except the block $U_{2}$, which contains arbitrary coordinates in place of the 0 block (cf. (3.20)). Then for $u_{0}^{\bullet} \in U_{0}^{\bullet}, u^{i}$ denotes the element obtained from $u_{0}^{\bullet}$ by zeroing out the coordinates in the blocks $U_{j}$ with $j \neq i$.

As in the proof of Lemma $27((3.19)-(3.21))$, we proceed by factoring the integral through $U_{R}$, to produce an intertwining operator. Then (3.34) equals

$$
\begin{aligned}
& \int_{U_{R} \backslash G} \int_{U_{R}} \phi^{\vee, 0}(z g) \int_{U_{0}^{\prime}} \int_{V^{3}} \int_{V^{3}} f_{\ldots}^{0}\left(\delta_{0}\left(\operatorname{diag}\left(l_{a, b}, l_{a, b}\right) \delta_{1}\right) u_{0}^{\prime} \operatorname{diag}\left(I_{k n}, l_{a, b} v\right)(1, z g) \operatorname{diag}\left(l_{a, b} v^{\prime}, I_{k n}\right), s\right) \\
& \psi_{U}\left(u_{0}^{\prime}\right) d v d v^{\prime} d u_{0}^{\prime} d z d g .
\end{aligned}
$$

(Cf. (3.21).)

Let $z \in U_{R}$. First we conjugate $\operatorname{diag}\left(I_{k n}, V^{3}\right)$ by $(1, z)$. We can write

$$
\begin{aligned}
\operatorname{diag}\left(I_{k n}, l_{a, b} v\right)(1, z) & =\operatorname{diag}\left(I_{k n}, l_{a, b}\right) \operatorname{diag}\left(I_{k n}, v\right)(1, z) \\
& =\operatorname{diag}\left(I_{k n}, l_{a, b}\right)(1, z) \operatorname{diag}\left(I_{k n}, v_{z}\right) \operatorname{diag}\left(I_{k n}, v\right) \\
& =\left(\operatorname{diag}\left(I_{k n}, l_{a, b}\right)(1, z)\right) \operatorname{diag}\left(I_{k n}, l_{a, b}\right) \operatorname{diag}\left(I_{k n}, v_{z}\right) \operatorname{diag}\left(I_{k n}, v\right)
\end{aligned}
$$

with $v_{z} \in V_{(a, k n-a)}$. Then conjugating $\left(\operatorname{diag}\left(l_{a, b}, l_{a, b}\right) \delta_{1}\right) u_{0}^{\prime}$ by $\operatorname{diag}\left(I_{k n}, l_{a, b}\right)(1, z)$ we obtain $\operatorname{diag}\left(l_{a, b}, l_{a, b}\right) \delta_{1}$ multiplied by a general element of $U_{0}^{\bullet}$, and when we take $\operatorname{diag}\left(I_{k n}, l_{a, b}\right)(1, z)$ to the left and conjugate by $\delta_{0}$, and use the invariance properties of $W_{a}(\tau)$ on the top left $a \times a$ block $\left(W_{a}(\tau)\right.$ in the inducing data of $\left.f_{\ldots .}^{0}\right)$, we see that this element vanishes without emitting a character. 
Turning to $v_{z}$, the element $\operatorname{diag}\left(I_{k n}, l_{a, b}\right) \operatorname{diag}\left(I_{k n}, v_{z}\right)$ normalizes $U_{0}^{\bullet}$ with a change of variables that emits a character. This character is cancelled after we take $\operatorname{diag}\left(I_{k n}, l_{a, b}\right) \operatorname{diag}\left(I_{k n}, v_{z}\right)$ to the left: conjugate it by $\delta_{0}\left(\operatorname{diag}\left(l_{a, b}, l_{a, b}\right) \delta_{1}\right)$ and again use the invariance properties of $W_{a}(\tau)$. Also $\phi^{\vee, 0}(z g)=\phi^{\vee, 0}(g)$. Altogether (3.35) becomes

$$
\begin{aligned}
& \int_{U_{R} \backslash G} \phi^{\vee, 0}(g) \int_{U_{0}^{\bullet}} \int_{V^{3}} \int_{V^{3}} f_{\ldots}^{0}\left(\delta_{0}\left(\operatorname{diag}\left(l_{a, b}, l_{a, b}\right) \delta_{1}\right) u_{0}^{\bullet} \operatorname{diag}\left(I_{k n}, l_{a, b} v\right)(1, g) \operatorname{diag}\left(l_{a, b} v^{\prime}, I_{k n}\right), s\right) \\
& \psi_{U}\left(u_{0}^{\bullet}\right) d v d v^{\prime} d u_{0}^{\bullet} d g .
\end{aligned}
$$

Here the $d z$-integration was incorporated into $d u_{0}^{\bullet}$ (cf. (3.22)).

Put $\delta_{0}=w \operatorname{diag}\left(\delta_{0, a}, \delta_{0, b}\right) w^{\prime}$ with

$$
w=\left(\begin{array}{cccc}
I_{k a} & & & \\
& & I_{k b} & \\
& I_{k a} & & \\
& & & I_{k b}
\end{array}\right), \quad \delta_{0, a}=\left(\begin{array}{cc} 
& I_{k a} \\
I_{k a} &
\end{array}\right), \quad \delta_{0, b}=\left(\begin{array}{cc} 
& I_{k b} \\
I_{k b} &
\end{array}\right), \quad w^{\prime}=w^{-1} .
$$

Then

$$
\delta_{0} u_{0}^{\bullet}=w \cdot\left(\operatorname{diag}\left(\delta_{0, a}, \delta_{0, b}\right) w^{\prime}\right) u^{2} \cdot \operatorname{diag}\left(\delta_{0, a}, \delta_{0, b}\right) \cdot w^{\prime}\left(u^{1} u^{4}\right) \cdot w^{\prime} u^{3} .
$$

Also $\operatorname{diag}\left(\delta_{0, a}, \delta_{0, b}\right) \cdot w^{\prime}\left(\operatorname{diag}\left(l_{a, b}, l_{a, b}\right) \delta_{1}\right)=\operatorname{diag}\left(\delta_{a}^{\prime}, \delta_{b}^{\prime}\right)$ is the embedding in $M_{(2 k a, 2 k b)}$ of the elements $\delta$ corresponding to the $\mathrm{GL}_{a} \times \mathrm{GL}_{k}$ and $\mathrm{GL}_{b} \times \mathrm{GL}_{k}$ integrals. Let $U^{2}$ be the subgroup of elements $\left(\operatorname{diag}\left(\delta_{0, a}, \delta_{0, b}\right) w^{\prime}\right) u^{2}, U^{1}$ corresponding to $w^{\prime}\left(u^{1}\right), U^{4}$ corresponding to $w^{\prime}\left(u^{4}\right)$, and $U^{3}$ to $u^{3}$. For $h \in H$,

$$
\begin{aligned}
& \int_{U_{0}^{\bullet}} f_{\ldots}^{0}\left(\delta_{0}\left(\operatorname{diag}\left(l_{a, b}, l_{a, b}\right) \delta_{1}\right) u_{0}^{\bullet} h, s\right) \psi_{U}\left(u_{0}^{\bullet}\right) d u_{0}^{\bullet} \\
& =\int_{U^{3}} \int_{U^{4}} \int_{U^{1}} \int_{U^{2}} f_{\ldots}^{0}\left(w u^{2} \operatorname{diag}\left(\delta_{a}^{\prime}, \delta_{b}^{\prime}\right) u^{1} u^{4} w^{\prime} u^{3} h, s\right) \psi_{U}(u) d u^{2} d u^{1} d u^{4} d u^{3} .
\end{aligned}
$$

The $d u^{2}$-integration over $U^{2}=\operatorname{diag}\left(I_{k a}, V_{(k a, k b)}, I_{k b}\right)$ defines an intertwining operator $M(s)$ from the space of (3.31) to the space of

$$
\operatorname{Ind}_{P_{(a k, a k, b k, b k)}}^{H}\left(|\operatorname{det}|^{-b / 2+\alpha s} W_{a}(\tau) \otimes|\operatorname{det}|^{-b / 2-\alpha s} W_{a}\left(\tau^{\prime}\right) \otimes|\operatorname{det}|^{a / 2+\alpha s} W_{b}(\tau) \otimes|\operatorname{det}|^{a / 2-\alpha s} W_{b}\left(\tau^{\prime}\right)\right),
$$

applied to $f_{\ldots .}^{0}$. The result is a rational section in the space of $(3.37)$, such that for all $\operatorname{diag}(x, y) \in$ $M_{(2 k a, 2 k b)}$,

$$
\begin{aligned}
& M(s) f_{\ldots}^{0}(\operatorname{diag}(x, y), s) \\
& =d_{\tau, \tau^{\prime}, a, b}(s)|\operatorname{det} x|^{k b-b / 2}|\operatorname{det} y|^{-k a+a / 2} \rho_{W_{a}(\tau) \otimes W_{a}\left(\tau^{\prime}\right)}^{0}(x, \alpha s /(k a)) \varrho_{W_{b}(\tau) \otimes W_{b}\left(\tau^{\prime}\right)}^{0}(y, \alpha s /(k b)) .
\end{aligned}
$$

The powers of $|\operatorname{det} x|$ and $|\operatorname{det} y|$ will cancel out as we explain.

For $g=\operatorname{diag}(x, y) \in M_{R}$, conjugating $\operatorname{diag}\left(I_{k n}, V^{3}\right)$ by $(1, g)$ multiplies the measure by $|\operatorname{det} y|^{(k-1) a}$; conjugating $U^{3}$ by $\operatorname{diag}\left(I_{k n}, l_{a, b}\right)(1, g)$ multiplies the measure by $|\operatorname{det} x|^{(1-k) b}$; and when we use the Iwasawa decomposition $G=R K_{G}$ and consider the modulus character emitted by $\phi^{\vee, 0}$, the integrand is further multiplied by $\delta_{R}^{-1 / 2}(g)$; so that the total change of measure is $|\operatorname{det} x|^{-k b+b / 2}|\operatorname{det} y|^{k a-a / 2}$.

In addition,

$$
w^{\prime}\left(\operatorname{diag}\left(I_{k n}, l_{a, b}\right)(1, g)\right)=\operatorname{diag}\left(I_{k a}, x, I_{(k-1) a}, I_{k b}, y, I_{(k-1) b}\right)=(1, x)(1, y),
$$


where $(1, x)$ is the embedding of $\mathrm{GL}_{a}$ in the $\mathrm{GL}_{a} \times \mathrm{GL}_{k}$ integral on the top left block of $M_{(2 k a, 2 k b)}$, for the representations $\pi_{a} \times \tau$, and $(1, y)$ is the embedding corresponding to the $\mathrm{GL}_{b} \times \mathrm{GL}_{k}$ integral on the bottom right block of $M_{(2 k a, 2 k b)}$, for $\pi_{b} \times \tau$. Regarding the unipotent integrations over the copies of $V^{3}$ and over $U^{3}$, we can see (using conjugations as in $\S 3.5 .1$ ) that the $d u^{3}$ integral vanishes unless both copies of $V^{3}$ are in $K_{H}$, then the integral over $U^{3}$ also vanishes outside $U^{3} \cap K_{H}$, so that the integrals $d u^{3} d v d v^{\prime}$ evaluate to 1. Integral (3.36) is then equal to

$$
d_{\tau, \tau^{\prime}, a, b}(s) Z\left(\alpha s /(k a), \omega_{a}^{0}, \rho_{W_{a}(\tau) \otimes W_{a}\left(\tau^{\prime}\right)}^{0}\right) Z\left(\alpha s /(k b), \omega_{b}^{0}, \varrho_{W_{b}(\tau) \otimes W_{b}\left(\tau^{\prime}\right)}^{0}\right) .
$$

In conclusion,

$$
Z\left(s, \omega^{0}, f_{W_{n}(\tau) \otimes W_{n}\left(\tau^{\prime}\right)}^{0}\right)=d_{\tau, \tau^{\prime}, a, b}(s) Z\left(\alpha s /(k a), \omega_{a}^{0}, \rho_{W_{a}(\tau) \otimes W_{a}\left(\tau^{\prime}\right)}^{0}\right) Z\left(\alpha s /(k b), \omega_{b}^{0}, \varrho_{W_{b}(\tau) \otimes W_{b}\left(\tau^{\prime}\right)}^{0}\right) .
$$

Let us turn to $d_{\tau, \tau^{\prime}, a, b}(s)$. Put $H^{\prime}=\mathrm{GL}_{k n}$. Restricting the normalized unramified section in the space of (3.31) to the subgroup $\operatorname{diag}\left(I_{k a}, H^{\prime}, I_{k b}\right)$ of $H$, it becomes an unramified element in the space of the unramified principal series representation of $H^{\prime}$ induced from

$$
\left(\otimes_{1 \leq i \leq k, 1 \leq j \leq b} \chi_{i}||^{\alpha s+(a-b) / 2+j-1 / 2}\right) \otimes\left(\otimes_{1 \leq i^{\prime} \leq k, 1 \leq j^{\prime} \leq a} \chi_{i^{\prime}}^{\prime}||^{-\alpha s-n / 2+j^{\prime}-1 / 2}\right) .
$$

The adjoint action of $\mathrm{GL}_{k b}(\mathbb{C}) \times \mathrm{GL}_{k a}(\mathbb{C})$ on the Lie algebra $\operatorname{Mat}_{k b \times k a}(\mathbb{C})$ is given by $[A, B] \cdot T=$ $A T B^{-1}$. The value of $d_{\tau, \tau^{\prime}, a, b}(s)$ now follows as in $\S 3.5 .1$.

3.7. Proof of Proposition 34. Here $\pi$ is an unramified quasi-character of $G=\mathrm{GL}_{1}=F^{*}$. Since the $(k, 1)$ model $W_{1}(\tau)$ is simply the Whittaker model, we can in this section consider any irreducible generic unramified representations $\tau$ and $\tau^{\prime}$ of $\mathrm{GL}_{k}$ (e.g., non-unitary), such that their central characters are inverses of one another. Since $\tau$ is irreducible, $W_{1}(\tau)$ is isomorphic to $\tau$ (and similarly for $\tau^{\prime}$ ). For $k=1$ Proposition 34 was proved in [PSR87a, $\S 6.1$ ] (using [GJ72]). Henceforth assume $k>1$.

The proof of the proposition and in particular the proof of Claim 36 below, is based on the ideas of Soudry [Sou93, Sou95, Sou00] (in the context of Rankin-Selberg integrals for $\mathrm{SO}_{2 n+1} \times \mathrm{GL}_{k}$, see also [Kap13a] for the application of these ideas to Rankin-Selberg integrals for $\mathrm{SO}_{2 n} \times \mathrm{GL}_{k}$ ).

For the $\mathrm{GL}_{1} \times \mathrm{GL}_{k}$ integral, $H=\mathrm{GL}_{2 k}$ and $P=P_{(k, k)}$. Then $U_{P}=V_{(k, k)}$. The section $h \mapsto f_{W_{1}(\tau) \otimes W_{1}\left(\tau^{\prime}\right)}(h, s)$ is on

$$
\mathrm{I}\left(W_{1}(\tau), W_{1}\left(\tau^{\prime}\right), s\right)=\operatorname{Ind}_{P_{(k, k)}}^{\mathrm{GL}_{2 k}}\left(\left(W_{1}(\tau) \otimes W_{1}\left(\tau^{\prime}\right)\right) \delta_{P_{(k, k)}}^{s}\right) .
$$

We recall the definitions of $U, \psi_{U}$, the embedding $\left(g_{1}, g_{2}\right): \mathrm{GL}_{1} \times \mathrm{GL}_{1} \rightarrow \mathrm{GL}_{2 k}$ and subgroup $U_{0}<U$ from $\S 3.2$ (for $n=1$ ). Here $\left.U=V_{\left(1^{k-1}, 2,1^{k-1}\right.}\right)$ and

$$
\psi_{U}(u)=\psi\left(-\sum_{i=1}^{k-1} u_{i, i+1}+u_{k, k+2}-\sum_{i=1}^{k-2} u_{k+1+i, k+2+i}\right) .
$$

Note that $U$ is obtained from $N_{\mathrm{GL}_{2 k}}$ by removing the $(k, k+1)$-th coordinate, and $\psi_{U}$ is "almost" a generic character of $N_{\mathrm{GL}_{2 k}}$. For brevity, throughout this section we write a general element of $V_{(k, k)}$ in the form

$$
\left[\begin{array}{ll}
y & z \\
u & x
\end{array}\right]=\left(\begin{array}{cccc}
I_{k-1} & & y & z \\
& 1 & u & x \\
& & 1 & \\
& & & I_{k-1}
\end{array}\right) .
$$

Then $U_{0}$ is the subgroup of elements $\left\{\left[\begin{array}{ll}y & z \\ 0 & x\end{array}\right]\right\}$ with arbitrary $x, y$ and $z$, and $\psi_{U}\left(\left[\begin{array}{cc}y & z \\ 0 & x\end{array}\right]\right)=\psi\left(x_{1}\right)$, where $x_{1}$ is the leftmost coordinate of (the row) $x$. The measure $d u_{0}$ on $U_{0}$ is the product measure on the coordinates of $x, y$ and $z$ separately, e.g., regarding $y$ as an element of $F^{k-1}$. The 
product $\mathrm{GL}_{1} \times \mathrm{GL}_{1}$ is embedded in the diagonal torus of $\mathrm{GL}_{2 k}$ by $\left(g_{1}, g_{2}\right)=\operatorname{diag}\left(g_{1} I_{k}, g_{2}, g_{1} I_{k-1}\right)$; it normalizes $U$ and stabilizes $\psi_{U}$. Also

$$
\delta_{0}=\left(\begin{array}{c}
I_{k} \\
I_{k}
\end{array}\right), \quad \delta_{1}=\operatorname{diag}\left(I_{k-1},\left(\begin{array}{cc}
1 & 1 \\
1
\end{array}\right), I_{k-1}\right)=\left[\begin{array}{ll}
0 & 0 \\
1 & 0
\end{array}\right] .
$$

The $\mathrm{GL}_{1} \times \mathrm{GL}_{k}$ integral $Z\left(s, \omega, f_{W_{1}(\tau) \otimes W_{1}\left(\tau^{\prime}\right)}\right)$ takes the form

$$
\int_{F^{*}} \int f_{W_{1}(\tau) \otimes W_{1}\left(\tau^{\prime}\right)}\left(\delta_{0}\left[\begin{array}{cc}
y & z \\
1 & x
\end{array}\right] \operatorname{diag}\left(I_{k}, a, I_{k-1}\right), s\right) \psi\left(x_{1}\right) \omega(a) d x d y d z d^{*} a .
$$

(We multiplied $\delta_{1}$ by $u_{0} \in U_{0}$.) Here and below, the domains of integration for variables $\left[\begin{array}{ll}y & z \\ u & x\end{array}\right]$ are omitted for brevity; they are products of $F$ according to the dimensions of the variables. Since $\pi^{-1}$ is a quasi-character, we can replace the matrix coefficient $\omega$ with $\pi^{-1}$ in (3.39), and denote $Z\left(s, f_{W_{1}(\tau) \otimes W_{1}\left(\tau^{\prime}\right)}\right)=Z\left(s, \pi^{-1}, f_{W_{1}(\tau) \otimes W_{1}\left(\tau^{\prime}\right)}\right)$. This integral is absolutely convergent in a right half-plane which is independent of the choice of section, and in this domain it satisfies the following equivariance properties:

$$
Z\left(s,\left(g_{1}, g_{2}\right) u \cdot f_{W_{1}(\tau) \otimes W_{1}\left(\tau^{\prime}\right)}\right)=\psi_{U}^{-1}(u) \pi\left(g_{2}\right) \pi^{-1}\left(g_{1}\right) Z\left(s, f_{W_{1}(\tau) \otimes W_{1}\left(\tau^{\prime}\right)}\right), \quad \forall g_{1}, g_{2} \in \mathrm{GL}_{1}, u \in U .
$$

Therefore, in its domain of convergence it can be regarded as an element of

$$
\operatorname{Hom}_{\mathrm{GL}_{1} \times \mathrm{GL}_{1}}\left(J_{U, \psi_{U}^{-1}}\left(\mathrm{I}\left(W_{1}(\tau), W_{1}\left(\tau^{\prime}\right), s\right)\right), \pi^{-1} \otimes \pi\right) .
$$

(This is (3.3) for $n=1$.)

Lemma 35. For all but a finite set of values of $q^{-s}$, the space (3.41) is at most one-dimensional.

The proof of the lemma appears at the end of this section. The statement is valid also for $k=1$ (see Remark 38). There is a choice of section such that $Z\left(s, f_{W_{1}(\tau) \otimes W_{1}\left(\tau^{\prime}\right)}\right)$ is absolutely convergent for all $s$, and equals a nonzero constant (independent of $s$ ). To see this, take $f_{W_{1}(\tau) \otimes W_{1}\left(\tau^{\prime}\right)}$ such that $\delta_{0} \cdot f_{W_{1}(\tau) \otimes W_{1}\left(\tau^{\prime}\right)}$ is right-invariant by $\mathcal{N}$ and supported in $P^{\delta_{0}} \delta_{1} \mathcal{N}$, where $\mathcal{N}$ is a small compact open neighborhood of the identity in $\mathrm{GL}_{2 k}$. Together with Lemma 35, Bernstein's continuation principle (in [Ban98]) implies that (3.39) admits meromorphic continuation to a rational function in $q^{-s}$.

We compute (3.39) by comparing it to another integral defined using the Whittaker model of (3.38). First consider the Jaquet integral realizing the Whittaker functional on (3.38), defined by

$$
f_{W_{1}(\tau) \otimes W_{1}\left(\tau^{\prime}\right)} \mapsto \int f_{W_{1}(\tau) \otimes W_{1}\left(\tau^{\prime}\right)}\left(\delta_{0}\left[\begin{array}{ll}
y & z \\
u & x
\end{array}\right], s\right) \psi(u) d x d y d z d u .
$$

This integral is absolutely convergent for $\operatorname{Re}(s) \gg 0$ and admits analytic continuation to a function in $\mathbb{C}\left[q^{-s}, q^{s}\right]$ (for a rational section of (3.38), the continuation is in $\mathbb{C}\left(q^{-s}\right)$ ). For any fixed $s$, the Whittaker model of (3.38) consists of the Whittaker functions

$$
W_{f_{W_{1}(\tau) \otimes W_{1}\left(\tau^{\prime}\right)}}(h, s)=\int f_{W_{1}(\tau) \otimes W_{1}\left(\tau^{\prime}\right)}\left(\delta_{0}\left[\begin{array}{ll}
y & z \\
u & x
\end{array}\right] h, s\right) \psi(u) d x d y d z d u \quad\left(h \in \mathrm{GL}_{2 k}\right),
$$

where on the right hand side the integral is defined by analytic continuation.

For any representation $\vartheta$ of $\mathrm{GL}_{2 k}$, let $\vartheta^{*}$ be the representation on the same space of $\vartheta$, defined

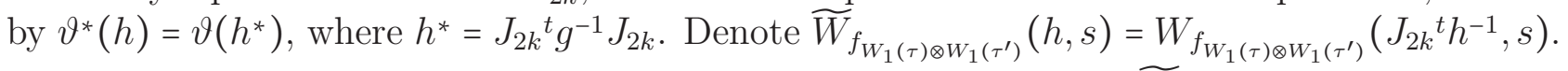
The Whittaker model of $\mathrm{I}\left(W_{1}(\tau), W_{1}\left(\tau^{\prime}\right), s\right)^{*}$ consists of the functions $\widetilde{W}_{f_{W_{1}(\tau) \otimes W_{1}\left(\tau^{\prime}\right)}}$ (see [JPSS83, 
$\S 2.1]$ ), again defined by analytic continuation. Also denote

$$
[t, v]=\operatorname{diag}\left(I_{k},\left(\begin{array}{ccc}
1 & & \\
& I_{k-2} & \\
-t & v & 1
\end{array}\right), \quad w^{\prime}=\operatorname{diag}\left(I_{k},\left({ }_{1} I_{k-1}\right)\right) .\right.
$$

Now consider the following integral

$$
\Psi\left(\zeta, s, f_{W_{1}(\tau) \otimes W_{1}\left(\tau^{\prime}\right)}\right)=\int_{F^{*}} \int_{F^{k-2}} \int_{F} W_{f_{W_{1}(\tau) \otimes W_{1}\left(\tau^{\prime}\right)}}\left(\operatorname{diag}\left(I_{2 k-1}, a\right)[t, v] w^{\prime}, s\right) \pi^{-1}(a)|a|^{\zeta+k-1} d t d v d^{*} a .
$$

Our first step is to show that for any fixed $s$, this integral is absolutely convergent in a left half-plane $\operatorname{Re}(\zeta) \ll 0$, and admits meromorphic continuation to (a function in) $\mathbb{C}\left(q^{-\zeta}\right)$. Observe that the integrand vanishes unless $t$ and $v$ belong to compact subgroups, independent of $a$ : if

$$
\mathrm{e}(\epsilon)=w^{\prime-1}\left(\begin{array}{cccc}
I_{k-1} & & & \\
& & & \\
& & I_{k-1} & \\
& & &
\end{array}\right)
$$

and $\epsilon$ is sufficiently small, depending on $f_{W_{1}(\tau) \otimes W_{1}\left(\tau^{\prime}\right)}$,

$$
\begin{aligned}
W_{f_{W_{1}(\tau) \otimes W_{1}\left(\tau^{\prime}\right)}}\left(\operatorname{diag}\left(I_{2 k-1}, a\right)[t, v] w^{\prime}, s\right) & =W_{f_{W_{1}(\tau) \otimes W_{1}\left(\tau^{\prime}\right)}}\left(\operatorname{diag}\left(I_{2 k-1}, a\right)[t, v] w^{\prime} \mathrm{e}(\epsilon), s\right) \\
& =\psi^{-1}(\epsilon t) W_{f_{W_{1}(\tau) \otimes W_{1}\left(\tau^{\prime}\right)}}\left(\operatorname{diag}\left(I_{2 k-1}, a\right)[t, v] w^{\prime}, s\right) .
\end{aligned}
$$

Hence if $t$ is large, the Whittaker function must vanish. Therefore (3.42) becomes a finite sum of integrals

$$
\int_{F^{*}} \int_{F^{k-2}} W_{f_{W_{1}(\tau) \otimes W_{1}\left(\tau^{\prime}\right)}^{(i)}}\left(\operatorname{diag}\left(I_{2 k-1}, a\right)[0, v] w^{\prime}, s\right) \pi^{-1}(a)|a|^{\zeta+k-1} d v d^{*} a,
$$

where $W_{f_{W_{1}(\tau) \otimes W_{1}\left(\tau^{\prime}\right)}^{(i)}}^{(a r e ~ W h i t t a k e r ~ f u n c t i o n s, ~ r i g h t-t r a n s l a t i o n s ~ o f ~} W_{f_{W_{1}(\tau) \otimes W_{1}\left(\tau^{\prime}\right)}}$. Next let

$$
\mathrm{e}\left(\epsilon_{1}, \ldots, \epsilon_{k-2}\right)={ }^{w^{\prime-1}} \operatorname{diag}\left(I_{k},\left(\begin{array}{ccccc}
1 & & & & \epsilon_{1} \\
& \ddots & & & \vdots \\
& & 1 & 0 & \epsilon_{k-2} \\
& & & 1 & 0 \\
& & & & 1
\end{array}\right),\right.
$$

and for a fixed $1 \leq i \leq k-2$ choose $\epsilon_{i}$ sufficiently small and $\epsilon_{j}=0$ for $j \neq i$. Then as with $t$ above, using $\mathrm{e}\left(\epsilon_{1}, \ldots, \epsilon_{k-2}\right)$ (instead of $\left.\mathrm{e}(\epsilon)\right)$ we handle the coordinates of $v$ from left to right, starting with $i=1$ up to $i=k-2$, and see that (3.43) becomes a finite sum of integrals

$$
\begin{aligned}
& \int_{F^{*}} W_{f_{W_{1}(\tau) \otimes W_{1}\left(\tau^{\prime}\right)}^{(j)}}^{(j)}\left(\operatorname{diag}\left(I_{2 k-1}, a\right), s\right) \pi^{-1}(a)|a|^{\zeta+k-1} d^{*} a \\
& =\int_{F^{*}} W_{f_{W_{1}(\tau) \otimes W_{1}\left(\tau^{\prime}\right)}^{(j)}}^{(j)}\left(\operatorname{diag}\left(I_{2 k-1}, a^{-1}\right), s\right) \pi(a)|a|^{1-k-\zeta} d^{*} a \\
& =\int_{F^{*}} \widetilde{W}_{f_{W_{1}(\tau) \otimes W_{1}\left(\tau^{\prime}\right)}^{(j)}}^{(j)}\left(\operatorname{diag}\left(a, I_{2 k-1}\right) J_{2 k}, s\right) \pi(a)|a|^{1-k-\zeta} d^{*} a .
\end{aligned}
$$

But each of these is a Rankin-Selberg integral for $\mathrm{GL}_{2 k} \times \mathrm{GL}_{1}$ and its convergence for $\operatorname{Re}(\zeta) \ll 0$ and continuation to $\mathbb{C}\left(q^{-\zeta}\right)$ was proved in [JPSS83] (in fact, already in [GJ72]). 
In its domain of convergence and in general by meromorphic continuation, $\Psi\left(\zeta, s, f_{W_{1}(\tau) \otimes W_{1}\left(\tau^{\prime}\right)}\right)$ satisfies equivariance properties similar to (3.40) but with $\pi$ replaced by ||$^{-\zeta} \pi$ :

$$
\Psi\left(\zeta, s,\left(g_{1}, g_{2}\right) u \cdot f_{W_{1}(\tau) \otimes W_{1}\left(\tau^{\prime}\right)}\right)=\psi_{U}^{-1}(u)\left|g_{2}\right|^{-\zeta} \pi\left(g_{2}\right)\left|g_{1}\right|^{\zeta} \pi^{-1}\left(g_{1}\right) \Psi\left(\zeta, s, f_{W_{1}(\tau) \otimes W_{1}\left(\tau^{\prime}\right)}\right) .
$$

Then for $\zeta=0$, the meromorphic continuation of $\Psi\left(\zeta, s, f_{W_{1}(\tau) \otimes W_{1}\left(\tau^{\prime}\right)}\right)$ belongs to $(3.41)$ and by Lemma 35 , it is proportional to the meromorphic continuation of $Z\left(s, f_{W_{1}(\tau) \otimes W_{1}\left(\tau^{\prime}\right)}\right)$.

Claim 36. Let $\operatorname{Re}(s) \ll 0$. Then

$$
\gamma\left(k s+1 / 2, \pi^{-1} \times \tau, \psi\right) Z\left(s, f_{W_{1}(\tau) \otimes W_{1}\left(\tau^{\prime}\right)}\right)=\Psi\left(0, s, f_{W_{1}(\tau) \otimes W_{1}\left(\tau^{\prime}\right)}\right) .
$$

Here $\gamma\left(k s+1 / 2, \pi^{-1} \times \tau, \psi\right)$ is the Rankin-Selberg $\gamma$-factor of $\pi^{-1} \times \tau$ ([JPSS83]).

The proof is given below. Since $\gamma\left(k s+1 / 2, \pi^{-1} \times \tau, \psi\right) \in \mathbb{C}\left(q^{-s}\right)$, we deduce that $\Psi\left(0, s, f_{W_{1}(\tau) \otimes W_{1}\left(\tau^{\prime}\right)}\right)$ admits meromorphic continuation to $\mathbb{C}\left(q^{-s}\right)$. Then (3.45) immediately holds as an identity in $\mathbb{C}\left(q^{-s}\right)$. Now we can compute $Z\left(s, f_{W_{1}(\tau) \otimes W_{1}\left(\tau^{\prime}\right)}^{0}\right)$ using $\Psi\left(0, s, f_{W_{1}(\tau) \otimes W_{1}\left(\tau^{\prime}\right)}^{0}\right)$. For the computation of the latter, we start with $\Psi\left(\zeta, s, f_{W_{1}(\tau) \otimes W_{1}\left(\tau^{\prime}\right)}^{0}\right)$ for $\operatorname{Re}(\zeta) \ll 0$, then take $\zeta=0$. Since $\tau$ and $\tau^{\prime}$ are irreducible, we can also take $s$ such that $(3.38)$ is irreducible (e.g., $\left.\operatorname{Re}(s) \ll 0\right)$.

Since $f_{W_{1}(\tau) \otimes W_{1}\left(\tau^{\prime}\right)}^{0}$ is unramified, it is invariant on the right with respect to $w^{\prime}$, and

$$
\Psi\left(\zeta, s, f_{W_{1}(\tau) \otimes W_{1}\left(\tau^{\prime}\right)}^{0}\right)=\int_{F^{*}} \int_{F^{k-2}} \int_{F} W_{f_{W_{1}(\tau) \otimes W_{1}\left(\tau^{\prime}\right)}^{0}}\left(\operatorname{diag}\left(I_{2 k-1}, a\right)[t, v], s\right) \pi^{-1}(a)|a|^{\zeta+k-1} d t d v d^{*} a .
$$

The $d t d v$-integration can be computed by arguing as above: using conjugations by $w^{\prime} \mathrm{e}(\epsilon)$, now with $\epsilon \in \mathcal{O}^{*}$ we see that the integrand vanishes unless $t \in \mathcal{O}$, and then since $f_{W_{1}(\tau) \otimes W_{1}\left(\tau^{\prime}\right)}^{0}$ is unramified, the integral $d t$ equals 1 . Similarly we show that the coordinates of $v$ belong in $\mathcal{O}$, and the integral over these coordinates equals 1 . Thus

$$
\Psi\left(\zeta, s, f_{W_{1}(\tau) \otimes W_{1}\left(\tau^{\prime}\right)}^{0}\right)=\int_{F^{*}} \widetilde{W}_{f_{W_{1}(\tau) \otimes W_{1}\left(\tau^{\prime}\right)}^{0}}\left(\operatorname{diag}\left(a, I_{2 k-1}\right), s\right) \pi(a)|a|^{1-k-\zeta} d^{*} a
$$

$(\operatorname{see}(3.44))$.

Next observe that since $f_{W_{1}(\tau) \otimes W_{1}\left(\tau^{\prime}\right)}^{0}$ is normalized and unramified and (3.38) is irreducible (by our choice of $s$ ), by the Casselman-Shalika formula [CS80] and [BZ77, Cas80],

$$
\widetilde{W}_{f_{W_{1}(\tau) \otimes W_{1}\left(\tau^{\prime}\right)}^{0}}\left(I_{2 k}, s\right)=W_{f_{W_{1}(\tau) \otimes W_{1}\left(\tau^{\prime}\right)}^{0}}\left(I_{2 k}, s\right)=L\left(2 k s+1, \tau \times \tau^{\prime \vee}\right)^{-1} \neq 0 .
$$

Also

$$
\mathrm{I}\left(W_{1}(\tau), W_{1}\left(\tau^{\prime}\right), s\right)^{*}=\operatorname{Ind}_{P_{(k, k)}}^{\mathrm{GL}_{2 k}}\left(|\operatorname{det}|^{k s} \tau^{\prime \vee} \otimes|\operatorname{det}|^{-k s} \tau^{\vee}\right)
$$

is irreducible unramified and generic. Then by [JS81, Proposition 2.3] (see also [GJ72, § 6]),

$$
\Psi\left(\zeta, s, f_{W_{1}(\tau) \otimes W_{1}\left(\tau^{\prime}\right)}^{0}\right)=\frac{L\left(-\zeta-k s+1 / 2, \pi \times \tau^{\vee}\right) L\left(-\zeta+k s+1 / 2, \pi \times \tau^{\prime \vee}\right)}{L\left(2 k s+1, \tau \times \tau^{\prime \vee}\right)} .
$$

Then we can take $\zeta=0$ in this equality. Since also by [GJ72, JS81, JPSS83],

$$
\gamma\left(k s+1 / 2, \pi^{-1} \times \tau, \psi\right)^{-1}=\frac{L\left(k s+1 / 2, \pi^{-1} \times \tau\right)}{L\left(-k s+1 / 2, \pi \times \tau^{\vee}\right)},
$$


Claim 36 implies

$$
\begin{aligned}
Z\left(s, \omega^{0}, f_{W_{1}(\tau) \otimes W_{1}\left(\tau^{\prime}\right)}^{0}\right) & =Z\left(s, f_{W_{1}(\tau) \otimes W_{1}\left(\tau^{\prime}\right)}^{0}\right) \\
& =\gamma\left(k s+1 / 2, \pi^{-1} \times \tau, \psi\right)^{-1} \Psi\left(0, s, f_{W_{1}(\tau) \otimes W_{1}\left(\tau^{\prime}\right)}^{0}\right) \\
& =\frac{L\left(k s+1 / 2, \pi^{-1} \times \tau\right) L\left(k s+1 / 2, \pi \times \tau^{\prime \vee}\right)}{L\left(2 k s+1, \tau \times \tau^{\prime \vee}\right)} .
\end{aligned}
$$

This completes the proof of the proposition.

Proof of Claim 36. We start with the left hand side of (3.45). We take $\operatorname{Re}(s) \gg 0$, where it is absolutely convergent. Put

$$
\jmath(t)=\operatorname{diag}\left(I_{k},\left(\begin{array}{cc}
1 & -t \\
1
\end{array}\right), I_{k-2}\right) .
$$

For fixed $u, t \in F$,

$$
\begin{aligned}
& \int f_{W_{1}(\tau) \otimes W_{1}\left(\tau^{\prime}\right)}\left(\delta_{0}\left[\begin{array}{ll}
y & z \\
u & x
\end{array}\right] \jmath(t), s\right) \psi\left(x_{1}\right) d x d y d z \\
& =\psi((u-1) t) \int f_{W_{1}(\tau) \otimes W_{1}\left(\tau^{\prime}\right)}\left(\delta_{0}\left[\begin{array}{ll}
y & z \\
u & x
\end{array}\right], s\right) \psi\left(x_{1}\right) d x d y d z .
\end{aligned}
$$

Since $\int_{F} \psi((u-1) t) d t=0$ unless $u=1$, when we apply this to $Z\left(s, f_{W_{1}(\tau) \otimes W_{1}\left(\tau^{\prime}\right)}\right)$ we obtain

$$
\int_{F^{*}} \int f_{W_{1}(\tau) \otimes W_{1}\left(\tau^{\prime}\right)}\left(\delta_{0}\left[\begin{array}{cc}
y & z \\
u & x
\end{array}\right] \jmath(t) \operatorname{diag}\left(I_{k}, a, I_{k-1}\right), s\right) \psi\left(x_{1}\right) \pi^{-1}(a) d x d y d z d t d u d^{*} a .
$$

For a Schwartz-Bruhat function $\phi$ on $F$, define

$$
\phi f_{W_{1}(\tau) \otimes W_{1}\left(\tau^{\prime}\right)}(h, s)=\int_{F} f_{W_{1}(\tau) \otimes W_{1}\left(\tau^{\prime}\right)}\left(h \jmath^{\prime}(r), s\right) \phi(r) d r, \quad \jmath^{\prime}(r)=\left[\begin{array}{ll}
0 & 0 \\
r & 0
\end{array}\right] .
$$

Also let $\widehat{\phi}$ be the Fourier transform of $\phi$, defined by $\widehat{\phi}(t)=\int_{F} \phi(r) \psi^{-1}(r t) d r$.

Formally, we can change the order of integration $d t d u \mapsto d u d t$ and consider the integral

$$
\begin{aligned}
& Z^{\prime}\left(s, f_{W_{1}(\tau) \otimes W_{1}\left(\tau^{\prime}\right)}\right) \\
& =\int_{F^{*}} \int f_{W_{1}(\tau) \otimes W_{1}\left(\tau^{\prime}\right)}\left(\delta_{0}\left[\begin{array}{ll}
y & z \\
u & x
\end{array}\right] \jmath(t) \operatorname{diag}\left(I_{k}, a, I_{k-1}\right), s\right) \psi\left(x_{1}\right) \pi^{-1}(a) d x d y d z d u d t d^{*} a .
\end{aligned}
$$

The convergence of $(3.47)$ is in the sense that

$$
\int_{F^{*}} \int_{F}\left|\int f_{W_{1}(\tau) \otimes W_{1}\left(\tau^{\prime}\right)}\left(\delta_{0}\left[\begin{array}{ll}
y & z \\
u & x
\end{array}\right] \jmath(t) \operatorname{diag}\left(I_{k}, a, I_{k-1}\right), s\right) \psi\left(x_{1}\right) \pi^{-1}(a) d x d y d z d u\right| d t d^{*} a<\infty .
$$

To see this, note that since $f_{W_{1}(\tau) \otimes W_{1}\left(\tau^{\prime}\right)}$ is locally constant on the right, one can always choose $\phi$ such that $\phi f_{W_{1}(\tau) \otimes W_{1}\left(\tau^{\prime}\right)}=f_{W_{1}(\tau) \otimes W_{1}\left(\tau^{\prime}\right)}$. Hence the left hand side of (3.48) becomes

$$
\int_{F^{*}} \int_{F}\left|\int f_{W_{1}(\tau) \otimes W_{1}\left(\tau^{\prime}\right)}\left(\delta_{0}\left[\begin{array}{cc}
y & z \\
u & x
\end{array}\right] \jmath(t) \operatorname{diag}\left(I_{k}, a, I_{k-1}\right) \jmath^{\prime}(r), s\right) \phi(r) \psi\left(x_{1}\right) \pi^{-1}(a) d r d x d y d z d u\right| d t d^{*} a .
$$

We can change the order of integration: first integrate over $x, y, z$ and $u$, and then over $r$ because $\phi$ is compactly supported and the integral over $\left[\begin{array}{ll}y & z \\ u & x\end{array}\right]$ is absolutely convergent (because $\operatorname{Re}(s) \gg 0$, see e.g., [Sou93, § 4.4-§ 4.6] and [Sou93, § 11.15, Lemma 1]). Therefore we can conjugate $f^{\prime}(r)$ to the left and after changing variables in $x_{1}$ and $u$, obtain $\psi^{-1}\left(a^{-1} r t\right)$. We then 
integrate first over $r$ to obtain $\widehat{\phi}\left(a^{-1} t\right)$, and change variables $t \mapsto a t$. Then $\jmath(t) \mapsto \jmath(a t)$ and $\jmath$ (at) $\operatorname{diag}\left(I_{k}, a, I_{k-1}\right)=\operatorname{diag}\left(I_{k}, a, I_{k-1}\right) \jmath(t)$. Integral (3.49) equals

$$
\int_{F^{*}} \int_{F}\left|\int f_{W_{1}(\tau) \otimes W_{1}\left(\tau^{\prime}\right)}\left(\delta_{0}\left[\begin{array}{cc}
y & z \\
u & x
\end{array}\right] \operatorname{diag}\left(I_{k}, a, I_{k-1}\right) \jmath(t), s\right) \widehat{\phi}(t) \psi\left(x_{1}\right) \pi^{-1}(a)\right| a|d x d y d z d u| d t d^{*} a .
$$

The $d t$-integration produces a finite sum of integrals, and each is bounded in $\operatorname{Re}(s) \gg 0$ (see [Sou93, § 4.4-§ 4.6]). This proves (3.48).

Then for $\operatorname{Re}(s) \gg 0$, integral (3.47) also belongs to (3.41) hence by Lemma 35, it is proportional to (3.46). The proportionality factor is 1. Indeed, repeating the manipulations above used for the proof of (3.48),

$$
\begin{aligned}
& Z^{\prime}\left(s, \phi f_{W_{1}(\tau) \otimes W_{1}\left(\tau^{\prime}\right)}\right) \\
& =\int_{F^{*}} \int_{F} \int f_{W_{1}(\tau) \otimes W_{1}\left(\tau^{\prime}\right)}\left(\delta_{0}\left[\begin{array}{cc}
y & z \\
u & x
\end{array}\right] \jmath(a t) \operatorname{diag}\left(I_{k}, a, I_{k-1}\right), s\right) \widehat{\phi}(t) \psi\left(x_{1}\right) \pi^{-1}(a)|a| d x d y d z d u d t d^{*} a .
\end{aligned}
$$

Changing $u \mapsto u+1$, conjugating $\jmath($ at $)$ to the left and changing variables in $x_{1}: x_{1} \mapsto x_{1}+(u+1)$ at and in $z$, and since $f_{W_{1}(\tau) \otimes W_{1}\left(\tau^{\prime}\right)}\left(\delta_{0} \jmath(a t) h, s\right)=\psi^{-1}(a t) f_{W_{1}(\tau) \otimes W_{1}\left(\tau^{\prime}\right)}\left(\delta_{0} h, s\right)$, we obtain

$$
\int_{F^{*}} \int_{F} \int f_{W_{1}(\tau) \otimes W_{1}\left(\tau^{\prime}\right)}\left(\delta_{0}\left[\begin{array}{cc}
y & z \\
u+1 & x
\end{array}\right] \operatorname{diag}\left(I_{k}, a, I_{k-1}\right), s\right) \widehat{\phi}(t) \psi(a u t) \psi\left(x_{1}\right) \pi^{-1}(a)|a| d x d y d z d u d t d^{*} a .
$$

Then integrating first over $t$ and since $\int_{F} \widehat{\phi}(t) \psi(a u t) d t=\phi(a u)$ by the Fourier inversion formula, the last integral equals

$$
\int_{F^{*}} \int_{F} \int f_{W_{1}(\tau) \otimes W_{1}\left(\tau^{\prime}\right)}\left(\delta_{0}\left[\begin{array}{cc}
y & z \\
u+1 & x
\end{array}\right] \operatorname{diag}\left(I_{k}, a, I_{k-1}\right), s\right) \phi(a u) \psi\left(x_{1}\right) \pi^{-1}(a)|a| d x d y d z d u d^{*} a .
$$

Noticing that $\left[\begin{array}{cc}y & z \\ u+1 & x\end{array}\right]=\left[\begin{array}{cc}y & z \\ 1 & x\end{array}\right] \jmath^{\prime}(u)$ and $\jmath^{\prime}(u) \operatorname{diag}\left(I_{k}, a, I_{k-1}\right)=\operatorname{diag}\left(I_{k}, a, I_{k-1}\right) \jmath^{\prime}(a u)$, and changing $u \mapsto a^{-1} u$, we arrive at $Z\left(s, \phi f_{W_{1}(\tau) \otimes W_{1}\left(\tau^{\prime}\right)}\right)$. Therefore in $\mathbb{C}\left(q^{-s}\right)$,

$$
Z\left(s, f_{W_{1}(\tau) \otimes W_{1}\left(\tau^{\prime}\right)}\right)=Z^{\prime}\left(s, f_{W_{1}(\tau) \otimes W_{1}\left(\tau^{\prime}\right)}\right) .
$$

Let $W \in W_{1}(\tau)$ and choose $f_{W_{1}(\tau) \otimes W_{1}\left(\tau^{\prime}\right)}$ such that $\delta_{0} \cdot f_{W_{1}(\tau) \otimes W_{1}\left(\tau^{\prime}\right)}$ is right-invariant by a small neighborhood of the identity $\mathcal{N}$ in $\mathrm{GL}_{2 k}$, supported in $P \mathcal{N}$, and such that for all $a \in \mathrm{GL}_{k}$, $\delta_{0} \cdot f_{W_{1}(\tau) \otimes W_{1}\left(\tau^{\prime}\right)}\left(\operatorname{diag}\left(a, I_{k}\right), s\right)=|\operatorname{det} a|^{k(s+1 / 2)} W(a)$. Now take a Schwartz-Bruhat function $\phi$ on $F$ such that for all $s$ and $h \in \mathrm{GL}_{2 k}$,

$$
\int_{F} f_{W_{1}(\tau) \otimes W_{1}\left(\tau^{\prime}\right)}(h \jmath(t), s) \widehat{\phi}(t) d t=f_{W_{1}(\tau) \otimes W_{1}\left(\tau^{\prime}\right)}(h, s) .
$$

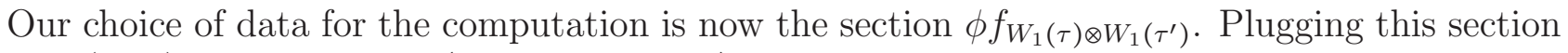
into (3.47), we see that $Z^{\prime}\left(s, \phi f_{W_{1}(\tau) \otimes W_{1}\left(\tau^{\prime}\right)}\right)$ equals

$$
\int_{F^{*}} \int f_{W_{1}(\tau) \otimes W_{1}\left(\tau^{\prime}\right)}\left(\delta_{0}\left[\begin{array}{cc}
y & z \\
u & x
\end{array}\right] \operatorname{diag}\left(I_{k}, a, I_{k-1}\right) \jmath(t) \jmath^{\prime}(r), s\right) \phi(r) \psi\left(x_{1}\right) \pi^{-1}(a)|a| d r d x d y d z d u d t d^{*} a .
$$

We can change the order of integration: first integrate over $x, y, z$ and $u$, and then over $r$ because $\phi$ is compactly supported. Therefore we can conjugate $\jmath^{\prime}(r)$ to the left and after changing variables in $x_{1}$ and $u$, obtain $\psi^{-1}(r t)$. Then we integrate first over $r$ to obtain $\widehat{\phi}(t)$. Now integrate over $t$, and by our choice of $\widehat{\phi}$ obtain

$$
\int_{F^{*}} \int f_{W_{1}(\tau) \otimes W_{1}\left(\tau^{\prime}\right)}\left(\delta_{0}\left[\begin{array}{ll}
y & z \\
u & x
\end{array}\right] \operatorname{diag}\left(I_{k}, a, I_{k-1}\right), s\right) \psi\left(x_{1}\right) \pi^{-1}(a)|a| d x d y d z d u d^{*} a .
$$


Conjugate $\operatorname{diag}\left(I_{k}, a, I_{k-1}\right)$ to the left. For our choice of $f_{W_{1}(\tau) \otimes W_{1}\left(\tau^{\prime}\right)}$ we see that the integrand vanishes unless the coordinates of $\left[\begin{array}{ll}y & z \\ u & x\end{array}\right]$ are small. Thus

$$
Z^{\prime}\left(s, \phi f_{W_{1}(\tau) \otimes W_{1}\left(\tau^{\prime}\right)}\right)=\int_{F^{*}} W\left(\operatorname{diag}\left(a, I_{k-1}\right)\right) \pi^{-1}(a)|a|^{k s+1 / 2-(k-1) / 2} d^{*} a,
$$

which is the Rankin-Selberg integral for $\mathrm{GL}_{1} \times \mathrm{GL}_{k}$ and $\pi^{-1} \times \tau_{0}([\mathrm{JPSS} 83, \S 2.4(3)]$ with $j=0)$. This integral is absolutely convergent for $\operatorname{Re}(s) \gg 0$, and admits meromorphic continuation to $\mathbb{C}\left(q^{-s}\right)$. Together with (3.50) we deduce, in $\mathbb{C}\left(q^{-s}\right)$ and in particular when $\operatorname{Re}(s) \ll 0$,

$$
Z\left(s, f_{W_{1}(\tau) \otimes W_{1}\left(\tau^{\prime}\right)}\right)=\int_{F^{*}} W\left(\operatorname{diag}\left(a, I_{k-1}\right)\right) \pi^{-1}(a)|a|^{k s+1 / 2-(k-1) / 2} d^{*} a .
$$

For the right hand side of $(3.45)$, since

$$
W_{\phi f_{W_{1}(\tau) \otimes W_{1}\left(\tau^{\prime}\right)}}(h, s)=\int_{F} W_{f_{W_{1}(\tau) \otimes W_{1}\left(\tau^{\prime}\right)}}\left(h \jmath^{\prime}(r), s\right) \phi(r) d r
$$

a similar (but simpler) computation shows, for $\operatorname{Re}(\zeta) \ll 0$,

$$
\Psi\left(\zeta, s, \phi f_{W_{1}(\tau) \otimes W_{1}\left(\tau^{\prime}\right)}\right)=\int_{F^{k-2}} \int_{F^{*}} W\left(\left(\begin{array}{ccc}
0 & 1 & 0 \\
0 & 0 & I_{k-2} \\
a & 0 & v
\end{array}\right)\right) \pi^{-1}(a)|a|^{\zeta+k s+1 / 2-(k-1) / 2} d^{*} a d v .
$$

This is again a Rankin-Selberg integral, now in the complex parameter $\zeta+k s$, which admits meromorphic continuation to $\mathbb{C}\left(q^{-\zeta-k s}\right)$ and is absolutely convergent when $\operatorname{Re}(s) \ll 0$ for any $\zeta$. Hence we can take $\zeta=0$ on the right hand side of (3.52) and obtain

$$
\int_{F^{k-2}} \int_{F^{*}} W\left(\left(\begin{array}{ccc}
0 & 1 & 0 \\
0 & 0 & I_{k-2} \\
a & 0 & v
\end{array}\right)\right) \pi^{-1}(a)|a|^{k s+1 / 2-(k-1) / 2} d^{*} a d v .
$$

Therefore when we take $\zeta=0$ on the left hand side of $(3.52)$, when $\operatorname{Re}(s) \ll 0$,

$$
\Psi\left(0, s, \phi f_{W_{1}(\tau) \otimes W_{1}\left(\tau^{\prime}\right)}\right)=\int_{F^{k-2}} \int_{F^{*}} W\left(\left(\begin{array}{ccc}
0 & 1 & 0 \\
0 & 0 & I_{k-2} \\
a & 0 & v
\end{array}\right)\right) \pi^{-1}(a)|a|^{k s+1 / 2-(k-1) / 2} d^{*} a d v .
$$

Finally (3.51) and (3.53) are related by $\gamma\left(s, \pi^{-1} \times \tau_{0}, \psi\right) \pi(-1)^{k-1}$ (see [Sou93, p. 70] for this version of [JPSS83, Theorem 2.7]), and $\pi(-1)=1$.

Remark 37. Alternatively, one can replace $\pi$ by ||$^{-\zeta} \pi$ throughout this section. Lemma 35 is then valid outside a finite set of $q^{-s}$ and $q^{-\zeta}$, Bernstein's continuation principle will imply that the integrals admit continuation to $\mathbb{C}\left(q^{-s}, q^{-\zeta}\right)$, and Claim 36 provides an identity in $\mathbb{C}\left(q^{-s}, q^{-\zeta}\right)$.

Proof of Lemma 35. The Jacquet module $J_{U, \psi_{U}^{-1}}\left(\mathrm{I}\left(W_{1}(\tau), W_{1}\left(\tau^{\prime}\right), s\right)\right)$ is a representation of the product $\mathrm{GL}_{1} \times \mathrm{GL}_{1}$, but since $\left\{\left(g_{1}, g_{1}\right): g_{1} \in F^{*}\right\}=C_{2 k}$ (the center of $\left.\mathrm{GL}_{2 k}\right)$, which acts trivially on $W_{1}(\tau) \otimes W_{1}\left(\tau^{\prime}\right)$ by our condition on $\tau$ and $\tau^{\prime}$ (their central characters are inverses of one another), it is natural to restrict our attention to one of the copies of $\mathrm{GL}_{1}$.

Identify $\mathrm{GL}_{1}$ with $\left\{\left(1, g_{2}\right): g_{2} \in F^{*}\right\}$, and in this manner regard $J_{U, \psi_{U}^{-1}}\left(\mathrm{I}\left(W_{1}(\tau), W_{1}\left(\tau^{\prime}\right), s\right)\right)$ as a representation of $\mathrm{GL}_{1}$. It is enough to prove the statement for

$$
\operatorname{Hom}_{\mathrm{GL}_{1}}\left(J_{U, \psi_{U}^{-1}}\left(\mathrm{I}\left(W_{1}(\tau), W_{1}\left(\tau^{\prime}\right), s\right)\right), \pi\right) .
$$


According to [BZ77, 1.9 (b), (d)], this space is isomorphic to

$$
\begin{aligned}
& \operatorname{Hom}_{\mathrm{GL}_{2 k}}\left(\mathrm{I}\left(W_{1}(\tau), W_{1}\left(\tau^{\prime}\right), s\right), \operatorname{Ind}_{\mathrm{GL}_{1} U}^{\mathrm{GL}_{2 k}}\left(\pi \otimes \psi_{U}^{-1}\right)\right) \\
& \cong \operatorname{Bil}_{\mathrm{GL}_{2 k}}\left(\operatorname{ind}_{\mathrm{GL}_{1} U}^{\mathrm{GL}_{2 k}}\left(\pi^{-1} \otimes \psi_{U}\right), \mathrm{I}\left(W_{1}(\tau), W_{1}\left(\tau^{\prime}\right), s\right)\right) .
\end{aligned}
$$

Here $\operatorname{Bil}(\cdots)$ is the space of $\mathrm{GL}_{2 k}$-equivariant bilinear forms and ind( $\left.\cdots\right)$ is the compact induction.

For $h \in P \backslash \mathrm{GL}_{2 k} / \mathrm{GL}_{1} U$ (a finite set), put

$$
\operatorname{Hom}(h)=\operatorname{Hom}_{\left(\mathrm{GL}_{1} U\right)^{h}}\left({ }^{h}\left(\pi^{-1} \otimes \psi_{U}\right) \otimes\left(W_{1}(\tau) \otimes W_{1}\left(\tau^{\prime}\right) \delta_{P}^{s}\right), \theta\right),
$$

where $\left(\mathrm{GL}_{1} U\right)^{h}={ }^{h}\left(\mathrm{GL}_{1} U\right) \cap P$; for a representation $\vartheta$ of $\mathrm{GL}_{1} U,{ }^{h} \vartheta$ is the representation of $\left(\mathrm{GL}_{1} U\right)^{h}$ on the space of $\vartheta$ given by ${ }^{h} \vartheta(x)=\vartheta\left({ }^{-1} x\right)$; and $\theta(x)=\delta_{\mathcal{C}(h)}\left(x,{ }^{h^{-1}} x\right) \delta_{P}^{-1 / 2}(x)$, where

$$
\mathcal{C}(h)=\left\{\left(x,{ }^{-1} x\right): x \in\left(\mathrm{GL}_{1} U\right)^{h}\right\}<P \times \mathrm{GL}_{1} U
$$

and $\delta_{\mathcal{C}(h)}$ is the modulus character of $\mathcal{C}(h)$. To us, the only important properties of $\theta$ are that it is independent of $s$ and trivial on unipotent elements (being a modulus character). Also note that by definition, the space of the representation on the left in $\operatorname{Hom}(h)$ is the space of $W_{1}(\tau) \otimes W_{1}\left(\tau^{\prime}\right)$.

According to the Bruhat theory (see e.g., [Sil79, Theorems 1.9.4 and 1.9.5], [Sou93, p. 48]), the space (3.54) injects into the semi-simplification

$$
\bigoplus_{h \in P \backslash \mathrm{GL}_{2 k} / \mathrm{GL}_{1} U} \operatorname{Hom}(h) .
$$

We may assume that a representative $h$ is either a permutation $w$ or $w \delta_{1}$, where $\delta_{1}=\left[\begin{array}{ll}0 & 0 \\ 1 & 0\end{array}\right]$, and recall $\delta_{0}=\left({ }_{I_{k}}{ }^{I_{k}}\right)$. Put $\kappa=\operatorname{diag}\left(I_{k-1},\left({ }_{1}{ }^{1}\right), I_{k-1}\right)$. Also write $h \sim h^{\prime}$ if $P h \mathrm{GL}_{1} U=P h^{\prime} \mathrm{GL}_{1} U$.

First assume $w \neq \delta_{0}$ and $w \neq \delta_{0} \kappa$. We claim that

$$
\left.\psi_{U}\right|_{h^{-1} V_{(k, k)} \cap U} \neq 1 .
$$

Granted this, we can choose $u \in h^{-1} V_{(k, k)} \cap U$ such that $\psi_{U}(u) \neq 1$. Then in $(3.55),{ }^{h}\left(\pi^{-1} \otimes\right.$ $\left.\psi_{U}\right)\left({ }^{h} u\right)=\psi_{U}(u) \neq 1$, and ${ }^{h} u$ acts trivially on $W_{1}(\tau) \otimes W_{1}\left(\tau^{\prime}\right)$ (because $\left.{ }^{h} u \in V_{(k, k)}\right)$. Thus the action of ${ }^{h} u$ on the left in (3.55) is nontrivial, while $\theta(u)=1$ on the right. This implies $\operatorname{Hom}(h)=0$.

We turn to prove (3.56). Note that ${ }^{h} V_{(k, k)}={ }^{w} V_{(k, k)}$. Write $w=\left(\begin{array}{ll}A_{1} & A_{2} \\ A_{3} & A_{4}\end{array}\right)$ with $A_{i} \in$ Mat $_{k}$. Since in particular $w \neq \delta_{0}$, we can assume $A_{1} \neq 0$. If the first column of $A_{1}$ is nonzero, let $i_{0}$ be the number of consecutive columns of $A_{1}$ starting from the first which are nonzero, so by definition $0<i_{0} \leq k$; if the first column of $A_{1}$ is zero, put $i_{0}=0$. Assume $i_{0}>0$. Then we can write (perhaps after multiplying $w$ by a permutation from $M_{P}=M_{(k, k)}$ )

$$
w=\left(\begin{array}{ccc}
I_{i_{0}} & & \\
& 0 & \\
& I_{i_{1}} & \\
& & \ddots
\end{array}\right),
$$

where the zero block above $I_{i_{1}}$ is the $\left(k-i_{0}\right) \times i_{1}$ zero matrix and $i_{1} \geq 1$.

For any $y \in F$, let $\jmath_{i, l}(y) \in N_{\mathrm{GL}_{2 k}}$ be such that its $(i, l)$-th coordinate is $y$, and the remaining coordinates above the diagonal are zero. We need to show that for some $i, l$ such that $j_{i, l}(y) \epsilon$ $V_{(k, k)}, h^{-1} \jmath_{i, l}(y)$ belongs to $U$ and $\psi_{U}\left(h^{-1} \jmath_{i, l}(y)\right) \neq 1$.

This is clear if $i_{0}=k$ (then we can take $w=I_{2 k}$ ), using $\jmath_{k, k+2}(y)$ (which commutes with $\delta_{1}$ ). If $i_{0}=k-1$, either $h^{-1} \jmath_{k-1, k+1}(y)=\jmath_{k-1, k}(y)$ if $h=w$, or when $h=w \delta_{1}$,

$$
h^{-1} \jmath_{k-1, k+1}(y)={ }^{\delta_{1}^{-1}} \jmath_{k-1, k}(y)=\jmath_{k-1, k}(y) \jmath_{k-1, k+1}(y) .
$$


In both cases we use $\jmath_{k-1, k+1}(y)$. Also if $0<i_{0}<k-1, h^{-1} \jmath_{i_{0}, k+1}(y)=\jmath_{i_{0}, i_{0}+1}(y)$. This verifies (3.56) when $i_{0}>0$.

When $i_{0}=0$, we let $0<i_{1}<k$ be the number of consecutive columns of $A_{1}$, starting from the first, which are zero $\left(A_{1} \neq 0\right.$ whence $\left.i_{1}<k\right)$. Then we write

$$
w=\left(\begin{array}{ccc}
0 & I_{i_{2}} & \\
\vdots & & \ddots \\
0 & & \\
I_{i_{1}} & & \\
& \ddots &
\end{array}\right)
$$

where $I_{i_{1}}$ starts at the $(k+1,1)$-th coordinate, $i_{2}>0$ is the number of consecutive nonzero columns of ( $\left.\begin{array}{ll}A_{1} & A_{2}\end{array}\right)$ starting from the $\left(i_{1}+1\right)$-th column, and $I_{i_{2}}$ begins at the $\left(1, i_{1}+1\right)$-th coordinate. Note that $i_{2} \leq k$ and $i_{1}+i_{2} \leq 2 k-1$ (because $i_{1}<k$ ).

Thus we can assume

$$
w=\left(\begin{array}{cccc}
0 & I_{i_{2}} & & \\
\vdots & 0 & & \\
0 & \vdots & & \\
I_{i_{1}} & 0 & & \\
& 0 & I_{i_{3}} & \\
& & & \ddots
\end{array}\right)
$$

where $i_{3} \geq 1$ and $I_{i_{3}}$ starts at the $\left(k+i_{1}+1, i_{1}+i_{2}+1\right)$-th coordinate. By matrix multiplication,

$$
w^{-1} \jmath_{i_{2}, k+i_{1}+1}(y)=\jmath_{i_{1}+i_{2}, i_{1}+i_{2}+1}(y) \text {. }
$$

If $i_{1}+i_{2} \geq k+2$ or $i_{1}+i_{2} \leq k-2$, then $\delta_{1}$ commutes with $j_{i_{1}+i_{2}, i_{1}+i_{2}+1}(y)$ and $\psi_{U}$ is nontrivial on $j_{i_{1}+i_{2}, i_{1}+i_{2}+1}(y)$, hence we can take $j_{i_{2}, k+i_{1}+1}(y)$. If $i_{1}+i_{2}=k+1$, then $i_{2}>1$ (since $i_{1}<k$ ) and $w^{-1} \jmath_{i_{2}-1, k+i_{1}+1}(y)=\jmath_{k, k+2}(y)$, so that we can take $\jmath_{i_{2}-1, k+i_{1}+1}(y)$. When $i_{1}+i_{2}=k-1$, $w^{-1} \jmath_{i_{2}, k+i_{1}+1}(y)=\jmath_{k-1, k}(y)$ and in both cases $\left(h=w\right.$ or $\left.h=w \delta_{1}\right), \psi_{U}$ is nontrivial on ${ }^{h^{-1}} \jmath_{i_{2}, k+i_{1}+1}(y)$ (as above, when $i_{0}=k-1$ ). If $i_{1}+i_{2}=k$ and $i_{3} \geq 2$, then again $h^{-1} \jmath_{i_{2}, k+i_{1}+2}(y)=\jmath_{k, k+2}(y)$.

The remaining case is $i_{1}+i_{2}=k$, in particular $i_{2}<k$, and $i_{3}=1$. In this case we further write

$$
w=\left(\begin{array}{cccccc}
0 & I_{i_{2}} & 0 & & & \\
\vdots & 0 & \vdots & I_{i_{4}} & & \\
0 & \vdots & & & \ddots & \\
I_{i_{1}} & 0 & & & & \\
& 0 & 1 & 0 & & \\
& & & 0 & I_{i_{5}} & \\
& & & & & \ddots
\end{array}\right),
$$

where $i_{4}>0$ (because $i_{2}<k$ ). If $i_{5}=0$, then $i_{1}=k-1$ whence $i_{2}=1$ and $i_{4}=k-1$, so that

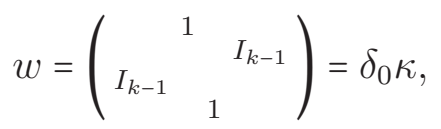

contradicting our assumption $\left(w \leftarrow \delta_{0} \kappa\right)$. Therefore $i_{5}>0$. Then $j_{i_{2}+i_{4}, k+i_{1}+2}(y) \in V_{(k, k)}$ because $i_{2}+i_{4} \leq k$ (also $k+i_{1}+2 \leq 2 k$ since $\left.k+i_{1}+1+i_{5} \leq 2 k\right), w^{-1} \jmath_{i_{2}+i_{4}, k+i_{1}+2}(y)=\jmath_{k+i_{4}+1, k+i_{4}+2}(y)$ which commutes with $\delta_{1}$ because $k+i_{4}+1 \geq k+2$, and $\psi_{U}\left(J_{k+i_{4}+1, k+i_{4}+2}(y)\right) \neq 1$. In this case take $J_{i_{2}+i_{4}, k+i_{1}+2}(y)$. This verifies (3.56) when $i_{0}=0$, completing all cases.

There are now three possibilities remaining for $h$ : $\delta_{0}, \delta_{0} \kappa$ or $\delta_{0} \delta_{1}$ (note that $\delta_{0} \kappa \sim \delta_{0} \kappa \delta_{1}$ ), because we proved $\operatorname{Hom}(h)=0$ in all other cases. 
Consider $h=\delta_{0}$. Then

$$
\left(\mathrm{GL}_{1} U\right)^{\delta_{0}}=\left({ }^{\delta_{0}} \mathrm{GL}_{1}\right) \ltimes\left(N_{\mathrm{GL}_{k}} \times N_{\mathrm{GL}_{k}}\right) .
$$

Moreover, if we write $\operatorname{diag}\left(x, I_{2 k-1}\right) \operatorname{diag}\left(v, v^{\prime}\right) \in\left(\mathrm{GL}_{1} U\right)^{\delta_{0}}$ where $v, v^{\prime} \in N_{\mathrm{GL}_{k}}$,

$$
{ }^{\delta_{0}}\left(\pi^{-1} \otimes \psi_{U}\right)\left(\operatorname{diag}\left(x, I_{2 k-1}\right) \operatorname{diag}\left(v, v^{\prime}\right)\right)=\pi^{-1}(x) \psi^{-1}\left(\sum_{i=1}^{k-1} v_{i, i+1}^{\prime}\right) \psi^{-1}\left(\sum_{i=2}^{k-1} v_{i, i+1}\right) .
$$

In particular ${ }^{\delta_{0}} \psi_{U}$ restricts to a degenerate character of the subgroup $\operatorname{diag}\left(N_{\mathrm{GL}_{k}}, I_{k}\right)$ of $\left(\mathrm{GL}_{1} U\right)^{\delta_{0}}$. Let $\mathcal{L} \in \operatorname{Hom}\left(\delta_{0}\right)$. For a pure tensor $\xi \otimes \xi^{\prime}$ in the space of $W_{1}(\tau) \otimes W_{1}\left(\tau^{\prime}\right)$ and $v \in V_{(1, k-1)}$, by (3.57),

$$
\mathcal{L}\left(W_{1}(\tau)(v) \xi \otimes \xi^{\prime}\right)=\mathcal{L}\left(W_{1}(\tau) \otimes W_{1}\left(\tau^{\prime}\right)\left(v, I_{k}\right) \xi \otimes \xi^{\prime}\right)=\mathcal{L}\left(\xi \otimes \xi^{\prime}\right) .
$$

Thus $\mathcal{L}$ factors through the Jacquet module $J_{V_{(1, k-1)}}\left(W_{1}(\tau)\right)$ of $W_{1}(\tau)$ along $V_{(1, k-1)}$. Moreover for $x \in \mathrm{GL}_{1}$,

$$
\mathcal{L}\left(\pi^{-1}(x)|x|^{k s} W_{1}(\tau) \otimes W_{1}\left(\tau^{\prime}\right)\left(\operatorname{diag}\left(x, I_{k-1}\right), I_{k}\right) \xi \otimes \xi^{\prime}\right)=\theta(x) \mathcal{L}\left(\xi \otimes \xi^{\prime}\right) .
$$

Hence

$$
\mathcal{L}\left(W_{1}(\tau)\left(\operatorname{diag}\left(x, I_{k-1}\right)\right) \xi \otimes \xi^{\prime}\right)=\pi(x)|x|^{-k s} \theta(x) \mathcal{L}\left(\xi \otimes \xi^{\prime}\right) .
$$

Since $\mathcal{L}$ must factor through one of the (finitely many) composition factors in a Jordan-Hölder series of $J_{V_{(1, k-1)}}\left(W_{1}(\tau)\right), W_{1}(\tau)\left(\operatorname{diag}\left(x, I_{k-1}\right)\right) \xi=\beta(x) \xi$ for some quasi-character $\beta$ of $F^{*}$, which belongs to a finite set of characters and is independent of $s$. We deduce

$$
\mathcal{L}\left(\xi \otimes \xi^{\prime}\right)=\beta^{-1}(x) \pi(x)|x|^{-k s} \theta(x) \mathcal{L}\left(\xi \otimes \xi^{\prime}\right) .
$$

Now if $\mathcal{L}$ is nonzero, it is nonzero on some $\xi \otimes \xi^{\prime}$, which may depend on $s$, but then

$$
|x|^{k s}=\beta^{-1}(x) \pi(x) \theta(x), \quad \forall x \in F^{*} .
$$

This equality can hold for at most finitely many values of $q^{-s}$. Therefore $\mathcal{L}=0$ and $\operatorname{Hom}\left(\delta_{0}\right)$ vanishes outside finitely many values of $q^{-s}$.

Assume $h=\delta_{0} \kappa$. In this case $\left(\mathrm{GL}_{1} U\right)^{\delta_{0} \kappa}={ }^{\delta_{0}} \kappa \mathrm{GL}_{1} \ltimes\left(N_{\mathrm{GL}_{k}} \times N_{\mathrm{GL}_{k}}\right)$ and (3.57) becomes

$$
{ }^{\delta_{0} \kappa}\left(\pi^{-1} \otimes \psi_{U}\right)\left(\operatorname{diag}\left(I_{2 k-1}, x\right) \operatorname{diag}\left(v, v^{\prime}\right)\right)=\pi^{-1}(x) \psi^{-1}\left(\sum_{i=1}^{k-2} v_{i, i+1}^{\prime}\right) \psi^{-1}\left(-v_{1,2}+\sum_{i=2}^{k-1} v_{i, i+1}\right) .
$$

Again ${ }^{\delta_{0} \kappa} \psi_{U}$ restricts to a degenerate character, now of $\operatorname{diag}\left(I_{k}, N_{\mathrm{GL}_{k}}\right)$. We can now argue as above: $\mathcal{L}$ factors through $J_{V_{(k-1,1)}}\left(W_{1}\left(\tau^{\prime}\right)\right)$ and instead of $(3.58)$ we have

$$
\mathcal{L}\left(W_{1}\left(\tau^{\prime}\right)\left(\operatorname{diag}\left(I_{k-1}, x\right)\right) \xi \otimes \xi^{\prime}\right)=\pi(x)|x|^{k s} \theta(x) \mathcal{L}\left(\xi \otimes \xi^{\prime}\right) .
$$

Thus $\operatorname{Hom}\left(\delta_{0} \kappa\right)$ vanishes outside finitely many values of $q^{-s}$.

Finally let $h=\delta_{0} \delta_{1}(h=\delta$ in the notation of $\S 3.2)$. Then $\left(\mathrm{GL}_{1} U\right)^{h}=N_{\mathrm{GL}_{k}} \times N_{\mathrm{GL}_{k}}$ and $\psi_{U}$ restricts to the non-degenerate character $\psi^{-1}(z)=\psi^{-1}\left(\sum_{i=1}^{k-1} z_{i, i+1}\right)$ on each $N_{\mathrm{GL}_{k}}$. Thus for $\mathcal{L} \in \operatorname{Hom}(h)$, a pure tensor $\xi \otimes \xi^{\prime}$ in the space of $W_{1}(\tau) \otimes W_{1}\left(\tau^{\prime}\right)$ and $v, v^{\prime} \in N_{\mathrm{GL}_{k}}$,

$$
\mathcal{L}\left(W_{1}(\tau) \otimes W_{1}\left(\tau^{\prime}\right)\left(v, v^{\prime}\right) \xi \otimes \xi^{\prime}\right)=\psi(v) \psi\left(v^{\prime}\right) \mathcal{L}\left(\xi \otimes \xi^{\prime}\right)
$$

so that $\mathcal{L}$ is in particular a Whittaker functional on $W_{1}(\tau) \otimes W_{1}\left(\tau^{\prime}\right) \cong \tau \otimes \tau^{\prime}$, and since $\tau$ and $\tau^{\prime}$ are irreducible generic, the functional $\mathcal{L}$ is unique up to scaling. 
Remark 38. For $k=1$ the proof of Lemma 35 is much simpler. First, the spaces Hom $(h)$ are a priori at most one-dimensional, because $\tau$ and $\tau^{\prime}$ are quasi-characters of $F^{*}$. It is therefore enough to show $\operatorname{Hom}(h)=0$ for $h \in\left\{\delta_{0}, I_{2}\right\}$ (now $\delta_{0} \kappa=I_{2}$ ), outside finitely many values of $q^{-s}$. For $h=\delta_{0}$ this follows immediately from (3.58), because now $W_{1}(\tau)(x)=\tau(x)$ (i.e., $\left.\beta=\tau\right)$, and for $h=I_{2}$ we use (3.59).

\section{REFERENCES}

[AGS15] A. Aizenbud, D. Gourevitch, and S. Sahi. Twisted homology for the mirabolic nilradical. Israel J. Math., 206(1):39-88, 2015.

[Ban98] W. D. Banks. A corollary to Bernstein's theorem and Whittaker functionals on the metaplectic group. Math. Res. Lett., 5(6):781-790, 1998.

[BB06] J. Beineke and D. Bump. A summation formula for divisor functions associated to lattices. Forum Math., 18(6):869-906, 2006.

[BZ77] I. N. Bernstein and A. V. Zelevinsky. Induced representations of reductive $p$-adic groups I. Ann. Scient. Éc. Norm. Sup., 10(4):441-472, 1977.

[BS00] S. Böcherer and C.-G. Schmidt. p-adic measures attached to Siegel modular forms. Ann. Inst. Fourier (Grenoble), 50(5):1375-1443, 2000.

[Bor79] A. Borel. Automorphic L-functions. In Automorphic Forms, Representations, and L-functions, volume 33 Part II, pages 27-61, 1979.

[Bum97] D. Bump. Automorphic forms and representations, volume 55 of Cambridge Studies in Advanced Mathematics. Cambridge University Press, Cambridge, 1997.

[BF90] D. Bump and S. Friedberg. The exterior square automorphic L-functions on GL(n). In Festschrift in honor of I. I. Piatetski-Shapiro on the occasion of his sixtieth birthday, Part II (Ramat Aviv, 1989), volume 3 of Israel Math. Conf. Proc., pages 47-65. Weizmann, Jerusalem, 1990.

[BG92] D. Bump and D. Ginzburg. Symmetric square L-functions on GL(r). Ann. of Math. (2), 136(1):137205, 1992.

[Cai18] Y. Cai. Fourier coefficients for degenerate Eisenstein series and the descending decomposition. Manuscripta Math., 156(3-4):469-501, 2018.

[CFGK] Y. Cai, S. Friedberg, D. Ginzburg, and E. Kaplan. Doubling Constructions for Covering Groups and Tensor Product L-Functions. Preprint 2016, available at http://arxiv.org/abs/1601.08240.

[CFK] Y. Cai, S. Friedberg, and E. Kaplan. Doubling constructions: local and global theory, with an application to global functoriality for non-generic cuspidal representations. Preprint 2018, available at https://arxiv.org/abs/1802.02637v2.

[Car93] R. W. Carter. Finite groups of Lie type. Wiley Classics Library. John Wiley \& Sons Ltd., Chichester, 1993. Conjugacy classes and complex characters, Reprint of the 1985 original, A Wiley-Interscience Publication.

[Cas80] W. Casselman. The unramified principal series of $p$-adic groups I: the spherical function. Compositio Math., 40(3):387-406, 1980.

[CS80] W. Casselman and J. A. Shalika. The unramified principal series of $p$-adic groups II: the Whittaker function. Compositio Math., 41(2):207-231, 1980.

[CKPSS04] J. W. Cogdell, H.H. Kim, I. I. Piatetski-Shapiro, and F. Shahidi. Functoriality for the classical groups. Publ. Math. Inst. Hautes Études Sci., 99(1):163-233, 2004.

[CPS94] J. W. Cogdell and I. I. Piatetski-Shapiro. Converse theorems for GL $_{n}$. Publ. Math. Inst. Hautes Études Sci., 79(1):157-214, 1994.

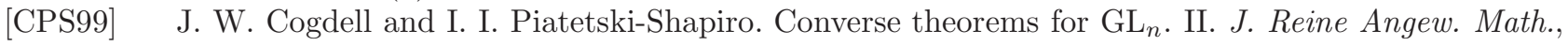
1999(507):165-188, 1999.

[CM93] D. H. Collingwood and W. M. McGovern. Nilpotent orbits in semisimple Lie algebras. Van Nostrand Reinhold Mathematics Series. Van Nostrand Reinhold Co., New York, 1993.

[DM78] J. Dixmier and P. Malliavin. Factorisations de fonctions et de vecteurs indéfiniment différentiables. Bull. Sci. Math. (2), 102(4):307-330, 1978.

[EHLS] E. Eischen, M. Harris, J.-S. Li, and C. M. Skinner. p-adic $L$-functions for unitary groups. Preprint 2016, available at http://arxiv.org/abs/1602.01776.

[FG16] S. Friedberg and D. Ginzburg. Criteria for the existence of cuspidal theta representations. Res. Number Theory, 2:2:16, 2016. 
[Gan12] W. T. Gan. Doubling zeta integrals and local factors for metaplectic groups. Nagoya Math. J., 208:67-95, 2012.

[GS12] W. T. Gan and G. Savin. Representations of metaplectic groups I: epsilon dichotomy and local Langlands correspondence. Compositio Math., 148:1655-1694, 2012.

[Gao18] F. Gao. The Langlands-Shahidi L-functions for Brylinski-Deligne extensions. Amer. J. Math., 140(1):83-137, 2018.

[Gar84] P. B. Garrett. Pullbacks of Eisenstein series; applications. In Automorphic forms of several variables (Katata, 1983), volume 46 of Progr. Math., pages 114-137. Birkhäuser Boston, Boston, MA, 1984.

[GPSR87] S. Gelbart, I. Piatetski-Shapiro, and S. Rallis. L-Functions for $G \times G L(n)$, volume 1254 of Lecture Notes in Math. Springer-Verlag, New York, 1987.

[Gin90] D. Ginzburg. L-functions for $\mathrm{SO}_{n} \times \mathrm{GL}_{k}$. J. Reine Angew. Math., 1990(405):156-180, 1990 .

[Gin03] D. Ginzburg. A construction of CAP representations in classical groups. Internat. Math. Res. Notices, 2003(20):1123-1140, 2003.

[Gin06] D. Ginzburg. Certain conjectures relating unipotent orbits to automorphic representations. Israel J. Math., 151(1):323-355, 2006.

[GJRS11] D. Ginzburg, D. Jiang, S. Rallis, and D. Soudry. L-functions for symplectic groups using FourierJacobi models. In Arithmetic geometry and automorphic forms, volume 19 of Adv. Lect. Math. (ALM), pages 183-207. Int. Press, Somerville, MA, 2011.

[GPSR97] D. Ginzburg, I. Piatetski-Shapiro, and S. Rallis. $L$ functions for the orthogonal group. Mem. Amer. Math. Soc., 128(611):viii+218, 1997.

[GRS98] D. Ginzburg, S. Rallis, and D. Soudry. L-functions for symplectic groups. Bull. Soc. math. France, 126:181-244, 1998.

[GRS99a] D. Ginzburg, S. Rallis, and D. Soudry. On a correspondence between cuspidal representations of $\mathrm{GL}_{2 n}$ and $\widehat{\mathrm{Sp}}_{2 n}$. J. Amer. Math. Soc., 12(3):849-907, 1999.

[GRS99b] D. Ginzburg, S. Rallis, and D. Soudry. On explicit lifts of cusp forms from $\mathrm{GL}_{m}$ to classical groups. Ann. of Math. (2), 150(3):807-866, 1999.

[GRS11] D. Ginzburg, S. Rallis, and D. Soudry. The descent map from automorphic representations of GL $(n)$ to classical groups. World Scientific Publishing, Singapore, 2011.

[GJ72] R. Godement and H. Jacquet. Zeta functions of simple algebras, volume 260 of Lecture Notes in Math. Springer-Verlag, Berlin, 1972.

[GGS] R. Gomez, D. Gourevitch, and S. Sahi. Whittaker supports for representations of reductive groups. Preprint 2016, available at http://arxiv.org/abs/1610.00284.

[GGS17] R. Gomez, D. Gourevitch, and S. Sahi. Generalized and degenerate Whittaker models. Compositio Math., 153(2):223-256, 2017.

[HKS96] M. Harris, S. S. Kudla, and W. J. Sweet. Theta dichotomy for unitary groups. J. Amer. Math. Soc., 9(4):941-1004, 1996.

[HLS05] M. Harris, J.-S. Li, and C. M. Skinner. The Rallis inner product formula and $p$-adic $L$-functions. In Automorphic representations, L-functions and applications: progress and prospects, volume 11 of Ohio State Univ. Math. Res. Inst. Publ., pages 225-255. de Gruyter, Berlin, 2005.

[HLS06] M. Harris, J.-S. Li, and C. M. Skinner. p-adic L-functions for unitary Shimura varieties. I. Construction of the Eisenstein measure. Doc. Math., Extra Vol.:393-464, 2006.

[Ike92] T. Ikeda. On the location of poles of the triple L-functions. Compositio Math., 83(2):187-237, 1992.

[Ike99] T. Ikeda. On the gamma factor of the triple $L$-function, I. Duke Math. J., 97(2):301-318, 1999.

[JL70] H. Jacquet and R. Langlands. Automorphic forms on GL(2). Lecture Notes in Mathematics, Vol. 114. Springer-Verlag, Berlin-New York, 1970.

[JPSS83] H. Jacquet, I. I. Piatetski-Shapiro, and J. A. Shalika. Rankin-Selberg convolutions. Amer. J. Math., 105(2):367-464, 1983.

[JR92] H. Jacquet and S. Rallis. Symplectic periods. J. Reine Angew. Math., 1992(423):175-197, 1992.

[JS90] H. Jacquet and J. Shalika. Exterior square L-functions. In Automorphic forms, Shimura varieties, and L-functions, Vol. II (Ann Arbor, MI, 1988), volume 11 of Perspect. Math., pages 143-226. Academic Press, Boston, MA, 1990.

[JS81] H. Jacquet and J. A. Shalika. On Euler products and the classification of automorphic representations I. Amer. J. Math., 103(3):499-558, 1981.

[JS83] H. Jacquet and J. A. Shalika. The Whittaker models of induced representations. Pacific. J. Math., 109(1):107-120, 1983. 
[JL13] D. Jiang and B. Liu. On Fourier coefficients of automorphic forms of GL(n). Internat. Math. Res. Notices, 2013(17):4029-4071, 2013.

[JZ14] D. Jiang and L. Zhang. A product of tensor product $L$-functions of quasi-split classical groups of Hermitian type. Geom. Funct. Anal., 24(2):552-609, 2014.

[Kap13a] E. Kaplan. Multiplicativity of the gamma factors of Rankin-Selberg integrals for $S O_{2 l} \times G L_{n}$. Manuscripta Math., 142(3-4):307-346, 2013.

[Kap13b] E. Kaplan. On the gcd of local Rankin-Selberg integrals for even orthogonal groups. Compositio Math., 149:587-636, 2013.

[Kap13c] E. Kaplan. On the local theory of Rankin-Selberg convolutions for $\mathrm{SO}_{2 l} \times \mathrm{GL}_{n}$. Thesis, Tel Aviv University, Israel, 2013.

[KM] E. Kaplan and J. Möllers. A Godement-Jacquet type integral and the metaplectic Shalika model. Preprint 2016, to appear in Amer. J. Math.

[Kim00] J. Kim. Gamma factors of certain supercuspidal representations. Math. Ann., 317(4):751-781, 2000.

[KR94] S. S. Kudla and S. Rallis. A regularized Siegel-Weil formula: the first term identity. Ann. of Math. (2), 140(1):1-80, 1994.

[Lan67] R. P. Langlands. Euler products. Yale University Press, New Haven, CT, 1967.

[Lan76] R. P. Langlands. On the functional equations satisfied by Eisenstein series. Lecture Notes in Mathematics, Vol. 544. Springer-Verlag, Berlin, 1976.

[LR05] E. M. Lapid and S. Rallis. On the local factors of representations of classical groups. In J. W. Cogdell, D. Jiang, S. S. Kudla, D. Soudry, and R. Stanton, editors, Automorphic representations, L-functions and applications: progress and prospects, pages 309-359. Ohio State Univ. Math. Res. Inst. Publ., 11, de Gruyter, Berlin, 2005.

[MW87] C. Mœglin and J.-L. Waldspurger. Modèles de Whittaker dégénérés pour des groupes p-adiques. Math. Z., 196(3):427-452, 1987.

[MW89] C. Møglin and J.-L. Waldspurger. Le spectre résiduel de GL(n). Ann. Sci. École Norm. Sup. (4), 22(4):605-674, 1989.

[MW95] C. Møeglin and J.-L. Waldspurger. Spectral decomposition and Eisenstein series, volume 113 of Cambridge Tracts in Mathematics. Cambridge University Press, Cambridge, 1995. Une paraphrase de l'Écriture [A paraphrase of Scripture].

[PSR86] I. Piatetski-Shapiro and S. Rallis. $\epsilon$ factor of representations of classical groups. Proc. Nat. Acad. Sci. U.S.A., 83(13):4589-4593, 1986.

[PSR87a] I. Piatetski-Shapiro and S. Rallis. L-functions for the classical groups, volume 1254 of Lecture Notes in Math. Springer-Verlag, New York, 1987.

[PSR87b] I. Piatetski-Shapiro and S. Rallis. Rankin triple $L$ functions. Compositio Math., 64(1):31-115, 1987.

[PS75] I. I. Piatetski-Shapiro. Euler subgroups. In Lie groups and their representations (Proc. Summer School, Bolyai János Math. Soc., Budapest, 1971), pages 597-620. Halsted, New York, 1975.

[RS05] S. Rallis and D. Soudry. Stability of the local gamma factor arising from the doubling method. Math. Ann., 333(2):291-313, 2005.

[Sat63] I. Satake. Theory of spherical functions on reductive algebraic groups over p-adic fields. Publ. Math. Inst. Hautes Études Sci., 18(1):5-69, 1963.

[Sha81] F. Shahidi. On certain L-functions. Amer. J. Math., 103(2):297-355, 1981.

[Sha90] F. Shahidi. A proof of Langlands' conjecture on Plancherel measures; complementary series for p-adic groups. Ann. of Math. (2), 132(2):273-330, 1990.

[Sha92] F. Shahidi. Twisted endoscopy and reducibility of induced representations for $p$-adic groups. Duke Math. J., 66(1):1-41, 1992.

[Sha74] J. A. Shalika. The multiplicity one theorem for $G L_{n}$. Ann. of Math., 100:171-193, 1974.

[Sil79] A. J. Silberger. Introduction to harmonic analysis on reductive p-adic groups. Princeton University Press and University of Tokyo Press, Princeton, New Jersey, 1979.

[Sou93] D. Soudry. Rankin-Selberg convolutions for $\mathrm{SO}_{2 l+1} \times \mathrm{GL}_{n}$ : local theory. Mem. Amer. Math. Soc., 105(500):vi+100, 1993.

[Sou95] D. Soudry. On the Archimedean theory of Rankin-Selberg convolutions for $\mathrm{SO}_{2 l+1} \times \mathrm{GL}_{n}$. Ann. Sci. École Norm. Sup. (4), 28(2):161-224, 1995.

[Sou00] D. Soudry. Full multiplicativity of gamma factors for $\mathrm{SO}_{2 l+1} \times \mathrm{GL}_{n}$. Israel J. Math., 120(1):511-561, 2000.

[Sou05] D. Soudry. On Langlands functoriality from classical groups to $\mathrm{GL}_{n}$. Astérisque, 298:335-390, 2005. 
[Sou17] D. Soudry. The unramified computation of Rankin-Selberg integrals expressed in terms of Bessel models for split orthogonal groups: part I. Israel J. Math., 222(2):711-786, 2017.

[Sou18] D. Soudry. The unramified computation of Rankin-Selberg integrals expressed in terms of Bessel models for split orthogonal groups: part II. J. Number Theory, 186:62-102, 2018.

[Spa82] N. Spaltenstein. Classes unipotentes et sous-groupes de Borel, volume 946 of Lecture Notes in Mathematics. Springer-Verlag, Berlin-New York, 1982.

[Tak97] K. Takano. On standard L-functions for unitary groups. Proc. Japan Acad. Ser. A Math. Sci., 73(1):5-9, 1997.

[Tak14] S. Takeda. The twisted symmetric square L-function of GL(r). Duke Math. J., 163(1):175-266, 2014.

[Wal03] J.-L. Waldspurger. La formule de Plancherel pour les groupes p-adiques, d'après Harish-Chandra. J. Inst. Math. Jussieu, 2(2):235-333, 2003.

[Yam14] S. Yamana. L-functions and theta correspondence for classical groups. Invent. Math., 196(3):651732,2014

[Zel80] A. V. Zelevinsky. Induced representations of reductive $p$-adic groups. II. On irreducible representations of GL(n). Ann. Sci. École Norm. Sup. (4), 13(2):165-210, 1980.

Friedberg: Department of Mathematics, Boston College, Chestnut Hill, MA 02467-3806, USA

E-mail address: solomon.friedberg@bc.edu

Cai: Department of Mathematics, Boston College, Chestnut Hill, MA 02467-3806, Usa

Current address: Department of Mathematics, Weizmann Institute of Science, Rehovot 7610001, ISRAEL

E-mail address: yuanqing.cai@weizmann.ac.il

Ginzburg: School of Mathematical Sciences, Tel Aviv University, Ramat Aviv, Tel Aviv 6997801, ISRAEL

E-mail address: ginzburg@post.tau.ac.il

Kaplan: Department of Mathematics, Bar Ilan University, Ramat Gan 5290002, Israel

E-mail address: kaplaney@gmail.com 